\title{
LIMIT THEOREMS FOR HOROCYCLE FLOWS
}

\author{
ALEXANDER BUFETOV AND GIOVANNI FORNI
}

\begin{abstract}
The main results of this paper are limit theorems for horocycle flows on compact surfaces of constant negative curvature.

One of the main objects of the paper is a special family of horocycle-invariant finitely additive Hölder measures on rectifiable arcs. An asymptotic formula for ergodic integrals for horocycle flows is obtained in terms of the finitely-additive measures, and limit theorems follow as a corollary of the asymptotic formula.

The objects and results of this paper are similar to those in [15], [16], [4] and $[5]$ for translation flows on flat surfaces. The arguments are based on the classification of invariant distributions for horocycle flows established in [12].
\end{abstract}

\section{CONTENTS}

1. Introduction

1.1. Outline of the main results.

1.2. Historical remarks.

1.3. Definitions and notation.

1.4. Hölder currents and Hölder cocycles.

1.5. Hölder cocycles and ergodic integrals.

1.6. Duality theorems.

1.7. Organization of the paper.

1.8. Acknowledgements

2. Basic Currents and Finitely-Additive Measures on Rectifiable Arcs.

2.1. Basic currents.

2.2. Geodesic scaling of basic currents.

2.3. Orthogonal projections on basic currents.

2.4. The construction of the finitely-additive measures.

2.5. Proof of the main properties (Theorem 1.1).

2.6. Proof of weak unstable vanishing (Corollary 1.1).

2.7. Proof of existence of dynamical projections (Corollary 1.2).

3. Additive Cocycles and Limit Distributions.

3.1. Proof of the cocycle theorem (Theorem 1.2).

3.2. Proof of the approximation theorem (Theorem 1.3).

3.3. Proof of the limit theorems: complementary series (Theorem 1.4).

3.4. Proof of the limit theorems: principal series (Theorem 1.5).

4. Duality and Classification.

4.1. Proof of the duality theorems (Theorem 1.6 and Theorem 1.7).

4.2. Proof of the classification theorem (Theorem 1.8).

4.3. $\Gamma$-invariant conformal distributions. 
4.4. Proof of the correspondence (Theorem 1.9).

5. Proofs of Technical Lemmas. 40

5.1. Outline of the section.

5.2. Estimates on coboundaries.

5.3. Inner products of cocycles.

5.4. Rotationally symmetric measures. 47

References 51

\section{INTRODUCTION}

1.1. Outline of the main results. The aim of this paper is to obtain limit theorems for horocycle flows on compact surfaces of constant negative curvature.

Our limit theorems admit the simplest formulation in the case when the smallest positive eigenvalue $\mu_{0}$ of the Laplace operator on the surface of curvature -1 is strictly less than $1 / 4$ (equivalently, when the spectral decomposition of the space of square-integrable functions on our surface into irreducible unitary representations of the modular group contains representations of the complementary series).

In this case, the variance of the ergodic integrals (up to time $T>0$ ) of a generic smooth function grows at the rate $T^{\frac{1+\nu_{0}}{2}}$, where $\nu_{0}:=\sqrt{1-4 \mu_{0}}$, and its ergodic integrals, normalized to have variance 1 , converge in distribution to a nondegenerate compactly supported measure on the real line.

The situation is more complicated for surfaces whose spectral decomposition only contains representations of the principal series (or more generally for functions supported on irreducible representations of the principal series).

In this case, the variance of ergodic integrals (up to time $T>0$ ) of any smooth function which is not a coboundary grows at the rate $T^{\frac{1}{2}}$, but its ergodic integrals, normalized to have variance 1 , converge in distribution to an orbit of an infinitedimensional quasi-periodic flow in the space of random variables with compactly supported distributions. The frequencies of this quasi-periodic motion are determined by the eigenvalues larger than $1 / 4$ of the Laplace-Beltrami operator on the hyperbolic surface. We are not able to determine whether the limit distribution exists in this case; we conjecture that it does not. In fact, the limit distribution will exist for all smooth functions which are not coboundaries if and only if all random variables in each of the invariant subtori of our infinite dimensional torus have exactly the same probability distribution (see $\S \S 5.4$ ).

Our argument relies on the classification, due to Flaminio and Forni [12], of distributions (in the sense of S. L. Sobolev and L. Schwart21 invariant under a given horocycle flow. One of the main objects of our paper is a closely related

\footnotetext{
${ }^{1}$ The term "distribution" is used in two very different senses in our paper: first, probability distributions of random variables and, second, distributions of Sobolev and Schwartz. For instance, "limit distributions" refer to the first meaning, while "invariant distributions" to the second. We hope that our precise meaning is always clear from the context.
} 
space of finitely-additive Hölder measures on rectifiable arcs on our surface, invariant under the complementary horocycle flow. We classify these measures and establish an explicit bijecitve correspondence between them and the subspace of the Flaminio-Forni space given by invariant distributions corresponding to positive eigenvalues of the Casimir operator. This isomorphism yields a natural duality between the spaces of invariant distributions for the two horocycle flows on a surface. We further establish an asymptotic formula for ergodic integrals in terms of the finitely-additive measures. Our limit theorems are obtained as corollaries of the asymptotic formula. Informally, the limit theorems claim that the normalized ergodic integrals of horocycle flows converge in distribution to the probability distributions of finitely-additive measures of horocycle arcs.

The objects and results of this paper are similar to those in [15], [16] and especially [4], [5], [6] for translation flows on flat surfaces. The methods here are completely different, however, and are based on those in [12].

The remainder of this section is organized as follows. In $\S \S 1.2$ we make some brief historical remarks. In $\S \S 1.3$ we establish our notation and recall the main properties of invariant distributions and basic currents for horocycle flows. In $\S \S 1.4$ we state our main theorems on finitely additive Hölder measures on rectifiable arcs, invariant with respect to the unstable (stable) horocycle (Theorem 1.1) and on the related additive cocycle for the stable (unstable) horocycle (Theorem 1.2). We also state several important corollaries of the above mentioned theorems (Corollary 1.1 and Corollary 1.2). In $\S \S 1.5$ we state our results on the asymptotics of ergodic integrals, in particular we state an approximation theorem for ergodic integrals of sufficiently smooth zero-average functions in terms of additive cocycles (Theorem 1.3) and our results on limit distributions of normalized ergodic integrals (Theorem 1.4 and Theorem 1.5). We then state our conditional results about the existence of limit distributions for functions supported on irreducible components of the principal series follow (Corollary 1.4 and Corollary 1.4). In $\S \S 1.6$ we introduce currents of dimension 2 (and degree 1) associated to our finitely additive measures on rectifiable arcs. We then state a duality theorem which affirms that such currents can be written in terms of invariant distributions for the unstable (stable) horocycle flow (Theorem 1.7). The duality theorem leads to a complete classification of the class of finitely additive Hölder measures axiomatically defined in $\S \S 1.4$ (see Definition 1.1), in the sense that our construction gives the space of all finitely additive Hölder measures with the listed properties (Theorem 1.8). It also allows us to establish a direct relations between the lifts of our additive cocyles to the universal cover and $\Gamma$-conformal invariant distributions on the boundary of the Poincaré disk (Theorem 1.9).

1.2. Historical remarks. The classical horocycle flow on a compact surface of constant negative curvature is a main example of a unipotent, parabolic flow. Its ergodic theory has been extensively studied. It is known that the flow is minimal [21], uniquely ergodic [17], has Lebesgue spectrum and is therefore strongly mixing [29], in fact mixing of all orders [27], and has zero entropy [20]. Its finer ergodic and rigidity properties, as well as the rate of mixing, were investigated by 
M. Ratner is a series of papers [30], [31], [32], [33]. In joint work with L. Flaminio [12], the second author has proved precise bounds on ergodic integrals of smooth functions. Those bounds already imply, as proved in [12], that all weak limits of probability distributions of normalized ergodic integrals of generic smooth functions have (non degenerate) compact support.

In the case of finite-volume surfaces, the classification of invariant measures is due to Dani [10]. The asymptotic behaviour of averages along closed horocycles in the finite-volume case has been studied by D. Zagier [36], P. Sarnak [34], D. Hejal [22] and more recently in [12] and by A. Strömbergsson [35]. The flows on general geometrically finite surfaces have been studied by M. Burger [7].

Invariant distributions, and, more generally, eigendistributions for smooth dynamical systems were already considered in 1955 by S. V. Fomin [14], who constructed a full system of eigendistributions for a linear toral automorphism.

In the case of horospherical foliations of symmetric spaces $X=G / K$ of noncompact type of connected semi-simple Lie groups $G$ with finite center, invariant distributions are related to conical distributions on the space of horocycles introduced in the work of S. Helgason [23].

Invariant distributions for the horocycle flow appear in the asymptotics for the equidistribution of long closed horocycle on finite-volume non-compact hyperbolic surfaces in work of P. Sarnak [34]. To the authors' best knowledge this is the first appearance of invariant distributions in the context of quantitative equidistribution. Sarnak's work was later generalized to arbitrary horocycle arcs and to the horocyle flow also in the compact case in [12] (see also [22], [35]).

Other similar (parabolic, uniquely ergodic) systems for which the asymptotics and the limit distributions of ergodic integrals have been studied include translation flows on surfaces of higher genus and interval exchange transformations, substitution dynamical systems and Vershik's automorphisms, and nilflows on homogeneous spaces of the Heisenberg group. The latter are related to the asymptotic behaviour of theta sums. For translation flows and interval exchange transformations, results on the growth of ergodic integrals were proved conditionally in the work of A. Zorich [37], [38], [39] and M. Kontsevich [26] and later fully proved in [16] and by A. Avila and M. Viana [1]. An asymptotic formula for ergodic integrals and limit theorems for translation flows were obtained in [4], [5] and [6]. Similar results for suspension flows over Vershik's automorphisms were obtained in [4]. Limit theorems for theta sums were proved by W. B. Jurkat and J. W. Van Horne [24], [25], by J. Marklof [28] and more recently in stronger form by F. Cellarosi [8]. Invariant distributions and asymptotics of ergodic integrals for Heisenberg nilflows were studied in [13], which generalizes the asymptotics for theta sums proved by H. Fiedler, W. B. Jurkat and O. Körner [11] .

1.3. Definitions and notation. Let $\Gamma$ be a co-compact lattice in $P S L(2, \mathbb{R})$ and let $M:=\Gamma \backslash D$ be the corresponding hyperbolic surface obtained as a quotient of the Poncaré disk $D$ under standard action of $\Gamma$ by linear fractional transformations. Since $P S L(2, \mathbb{R})$ acts freely and transitively on the unit tangent bundle of the Poncaré disk, the unit tangent bundle $S M$ of the hyperbolic surface $M$ can be 
identified with the homogeneous space $\Gamma \backslash P S L(2, \mathbb{R})$. Let $\{X, U, V\}$ be the basis of the Lie algebra $\mathfrak{s l}(2, \mathbb{R})$ of $P S L(2, \mathbb{R})$ given by the infinitesimal generators of the geodesic flow and of the stable and unstable horocycle flows. The following commutation relations hold:

$$
[X, U]=U, \quad[X, V]=-V, \quad[U, V]=2 X .
$$

Let $\{\hat{X}, \hat{U}, \hat{V}\}$ be the frame of the cotangent bundle dual to the frame $\{X, U, V\}$ of the tangent bundle, that is,

$$
\begin{array}{lll}
\hat{X}(X)=1, & \hat{X}(U)=0 & \hat{X}(V)=0 ; \\
\hat{U}(X)=0, & \hat{U}(U)=1 & \hat{U}(V)=0 ; \\
\hat{V}(X)=0, & \hat{V}(U)=0 & \hat{V}(V)=1 .
\end{array}
$$

Let $|\hat{X}|,|\hat{U}|$ and $|\hat{V}|$ denote the 1-dimensional measures on $S M$ tranverse to the 2-dimensional foliations $\{\hat{X}=0\},\{\hat{U}=0\}$ and $\{\hat{V}=0\}$ given respectively by the 1 -forms $\hat{X}, \hat{U}$ and $\hat{V}$. In other terms, if $\gamma$ is any rectifiable path transverse to the foliation $\{\hat{X}=0\},(\{\hat{U}=0\},\{\hat{V}=0\})$, then respectively,

$$
\int_{\gamma}|\hat{X}|=\left|\int_{\gamma} \hat{X}\right| \quad\left(\int_{\gamma}|\hat{U}|=\left|\int_{\gamma} \hat{U}\right|, \quad \int_{\gamma}|\hat{V}|=\left|\int_{\gamma} \hat{V}\right|\right) .
$$

Let $g_{t}:=\exp (t X), h_{t}^{U}=\exp (t U)$ and $h_{t}^{V}=\exp (t V)$ be the corresponding one-paramer groups. Since $P S L(2, \mathbb{R})$ acts on $S M$ on the right, the following commutation relations hold for the flows:

$$
g_{t} \circ h_{s}^{U}=h_{e^{-t} s}^{U} \circ g_{t} \quad \text { and } \quad g_{t} \circ h_{s}^{V}=h_{e^{t} s}^{U} \circ g_{t}, \quad \text { for all } s, t \in \mathbb{R} .
$$

Thus the flows $\left\{h_{t}^{U}\right\}$ and $\left\{h_{t}^{V}\right\}$ are respectively the stable and unstable horocycle flows for the hyperbolic geodesic flow $\left\{g_{t}\right\}$ on $S M$.

Let $L^{2}(S M)$ be the Hilbert space of square-integrable complex-valued functions on $S M$, endowed with its usual Hilbert space norm $\|\cdot\|$. By the theory of unitary representations of the unimodular group (see for instance [2], [18] [19]), the Hilbert space $L^{2}(S M)$ of splits as an orthogonal sum

$$
L^{2}(S M)=\bigoplus_{\mu \in \operatorname{Spec}(\square)} H_{\mu}
$$

of irreducible unitary representations of $\operatorname{PSL}(2, \mathbb{R})$ which are parametrized by the value $\mu \in \mathbb{R}^{+} \cup\left\{-n^{2}+n \mid n \in \mathbb{Z}^{+}\right\}$of the Casimir operator

$$
\square:=X^{2}+X+V U=X^{2}-X+U V=X^{2}+U V+V U .
$$

The Casimir operator is a second order differential operator which generates the center of the enveloping algebra of the Lie algebra $\mathfrak{s l}(2, \mathbb{R})$, hence its restriction to every irreducible unitary representation is a scalar multiple of the identity. The unitary type of irreducible unitary representations of $P S L(2, \mathbb{R})$ is uniquely determined by the value of the Casimir parameter. Irreducible unitary representations are divided into three series: the principal series consists of all representations with Casimir parameter $\mu \geq 1 / 4$, the complementary series of all representations with 
Casimir parameter $0<\mu<1 / 4$ and the discrete series of representations with Casimir parameter $\mu=-n^{2}+n$.

Remark. In formula (4) and everywhere below the eigenvalues of the Casimir operator are always understood with multiplicities.

Let us consider the stable horocycle flow $\left\{h_{t}^{U}\right\}$. Similar statements hold for the unstable horocycle flow. It was proved in [12] that for every Casimir parameter $\mu \in \mathbb{R}^{+}$the space of invariant distributions for the horocycle flow which are nontrivial on the space $C^{\infty}\left(H_{\mu}\right):=C^{\infty}(S M) \cap H_{\mu}$ has dimension equal to 2 and (if $\mu \neq 1 / 4)$ it is generated by eigenvector for the action of the geodesic flow.

We recall that an invariant distribution for the stable horocycle flow is a distribution $D \in \mathcal{D}^{\prime}(S M)$ such that $\mathcal{L}_{U} D=0$ in the sense of distributions. Let $\mathcal{J}_{U}(S M) \subset \mathcal{D}^{\prime}(S M)$ denote the space of all invariant distributions for the stable horocycle flow.

For every Casimir parameter $\mu \in \mathbb{R}^{+}$let $\nu:=\sqrt{1-4 \mu}$. We remark that $\nu \in \mathbb{C}$ is purely imaginary if $\mu \geq 1 / 4$ (principal series) and $\nu \in(0,1)$ if $0<\mu<$ $1 / 4$ (complementary series). By [12], Theorem 3.2 and Lemma 3.5, the space of invariant distributions for the stable horocycle flow which are non-trivial on $C^{\infty}\left(H_{\mu}\right)$ has a basis $\left\{D_{\mu}^{+}, D_{\mu}^{-}\right\}$such that (in the sense of distributions)

$$
\mathcal{L}_{X} D_{\mu}^{ \pm}=-\frac{1 \pm \nu}{2} D_{\mu}^{ \pm}, \quad \text { for all } \mu \in \mathbb{R}^{+} \backslash\{1 / 4\},
$$

while for the for the special case $\mu=1 / 4(\nu=0)$,

$$
\mathcal{L}_{X}\left(\begin{array}{l}
D_{\mu}^{+} \\
D_{\mu}^{-}
\end{array}\right)=-\frac{1}{2}\left(\begin{array}{ll}
1 & 0 \\
1 & 1
\end{array}\right)\left(\begin{array}{l}
D_{\mu}^{+} \\
D_{\mu}^{-}
\end{array}\right) .
$$

Invariant distributions for the horocycle flow are naturally associated (by a general construction which holds for any volume preserving flow) with basic currents for the horocycle foliation. A current $C$ of degree 2 (and dimension 1 ) is called basic for the orbit foliation of the stable horocycle flow if and only if

$$
\mathcal{L}_{U} C=\imath_{U} C=0 \text { in } \mathcal{D}^{\prime}(S M) .
$$

(The operators $\mathcal{L}_{U}$ and $\imath_{U}$ are respectively the Lie derivative and the contraction with respect to the horocycle generator $U$ acting on currents according to the standard definition). Let $\mathcal{B}_{U}(S M)$ denote the space of all basic currents for the stable horocycle foliation.

For any $s>0$, let $W^{s}(S M)$ and $\Omega_{1}^{s}(S M)$ be respectively the $L^{2}$ Sobolev spaces of functions and of 1-forms on $S M$ and let $W^{-s}(S M)$ and $\Omega_{1}^{-s}(S M)$ denote the dual Sobolev spaces of distributions. Let then

$$
\begin{aligned}
\mathcal{J}_{U}^{-s}(S M) & :=\mathcal{J}_{U}(S M) \cap W^{-s}(S M), \\
\mathcal{B}_{U}^{-s}(S M) & :=\mathcal{B}_{U}(S M) \cap \Omega_{1}^{-s}(S M) .
\end{aligned}
$$

Let $\omega$ denote the volume form on $S M$ and let $\eta_{U}:=\imath_{U} \omega$ denote the contraction of the volume form along the stable horocycle. The 2 -form $\eta_{U}$ is closed since the horocycle flow is volume preserving. We will show in Lemma 2.1 below that the 
space $\mathcal{J}_{U}(S M)$ of all invariant distributions and the space $\mathcal{B}_{U}(S M)$ are identified by the isomorphism

$$
D \rightarrow D \eta_{U}
$$

which maps the Sobolev space $\mathcal{J}_{U}^{-s}(S M)$ isometrically onto the Sobolev space $\mathcal{B}_{U}^{-s}(S M)$, for all $s>0$, hence it maps $\mathcal{J}_{U}(S M)$ onto $\mathcal{B}_{U}(S M)$.

It was proved in [12], 33.2 , that the invariant distributions $D_{\mu}^{ \pm}$have Sobolev orders equal to $(1 \pm \operatorname{Re} \nu) / 2$ (that is, $D_{\mu}^{ \pm} \in W^{-s}(S M)$ for all $\left.s>(1 \pm \operatorname{Re} \nu) / 2\right)$ ). It was later proved by S. Cosentino in [9] that $D_{\mu}^{ \pm}$are in fact Hölder of the same orders (that is, they can be written as first derivatives of Hölder continuous functions of exponent $1 \mp \operatorname{Re} \nu) / 2$, except for the distribution $D_{1 / 4}^{-}$which can be written as a first derivative of a Hölder continuous function of any exponent $\alpha<1 / 2$ ).

Notation: The Lie derivative $\mathcal{L}_{W}$ of a distribution or a current with respect to a smooth vector field $W$ is defined in the standard weak sense (based on the formula of integration by parts). For consistence, the action of a smooth flow $\left\{\phi_{t}\right\}$ on a current $C$ is defined by pull-back as follows:

$$
\left(\phi_{t}^{*} C\right)(\lambda)=C\left(\phi_{-t}^{*} \lambda\right), \quad \text { for any smooth form } \lambda .
$$

In particular, with the above convention the following identity holds. Let $W$ be the infinitesimal generator of the smooth flow $\left\{\phi_{t}\right\}$. For all $t \in \mathbb{R}$,

$$
\frac{d}{d t} \phi_{t}^{*} C=\phi_{t}^{*} \mathcal{L}_{W} C
$$

1.4. Hölder currents and Hölder cocycles. One of the main objects of this paper is a space of finitely-additive Hölder measures defined on the semi-ring of all rectifiable arcs in $S M$. These measures are invariant under the unstable horocycle flow $h_{t}^{V}$ and will be seen to govern the asymptotics of ergodic integrals for the stable horocycle flow $h_{t}^{U}$.

Definition 1.1. Let $\hat{\mathfrak{B}}_{V}(S M)$ be the space of all functionals $\hat{\beta}$ which to every rectifiable arc $\gamma \subset S M$ assign a complex number $\hat{\beta}(\gamma) \in \mathbb{C}$ so that the following holds:

(1) (Additive property) For any decomposition $\gamma=\gamma_{1}+\gamma_{2}$ into subarcs,

$$
\hat{\beta}(\gamma)=\hat{\beta}\left(\gamma_{1}\right)+\hat{\beta}\left(\gamma_{2}\right)
$$

(2) (Weak unstable vanishing) For all $\gamma$ tangent to the weak unstable foliation,

$$
\hat{\beta}(\gamma)=0 .
$$

(3) (Unstable horocycle invariance) For all $t \in \mathbb{R}$,

$$
\hat{\beta}\left(h_{t}^{V} \gamma\right)=\beta(\gamma)
$$

(4) (Hölder property) There exists an exponent $\alpha \in(0,1)$ and a constant $C>$ 0 such that for all rectifiable arcs $\gamma$ satisfying

$$
\int_{\gamma}|\hat{U}| \leq 1
$$


we have

$$
|\hat{\beta}(\gamma)| \leq C\left(1+\int_{\gamma}|\hat{X}|+\int_{\gamma}|\hat{U}| \int_{\gamma}|\hat{V}|\right)\left(\int_{\gamma}|\hat{U}|\right)^{\alpha} .
$$

The space $\hat{\mathfrak{B}}_{V}(S M)$ contains a sequence of special elements

$$
\left\{\hat{\beta}_{\mu}^{ \pm} \mid \mu \in \operatorname{Spec}(\square) \cap \mathbb{R}^{+}\right\}
$$

described in the following Theorem. We prove below that the above set is in fact a basis of $\hat{\mathfrak{B}}_{V}(S M)$ endowed with a natural Sobolev-type Hilbert space structure.

Theorem 1.1. For any positive Casimir parameter $\mu>0$ there exist two independent (normalized) finitely-additive measures $\hat{\beta}_{\mu}^{ \pm}$such that the following holds.

For all rectifiable arcs $\gamma$ in $S M$ the following properties hold:

(1) (Additive property) For any decomposition $\gamma=\gamma_{1}+\gamma_{2}$ into subarcs,

$$
\hat{\beta}_{\mu}^{ \pm}(\gamma)=\hat{\beta}_{\mu}^{ \pm}\left(\gamma_{1}\right)+\hat{\beta}_{\mu}^{ \pm}\left(\gamma_{2}\right)
$$

(2) (Geodesic scaling) For all $t \in \mathbb{R}$ and for $\mu \neq 1 / 4$,

$$
\hat{\beta}_{\mu}^{ \pm}\left(g_{-t} \gamma\right)=\exp \left(\frac{1 \mp \nu}{2} t\right) \hat{\beta}_{\mu}^{ \pm}(\gamma)
$$

while for $\mu=1 / 4(\nu=0)$,

$$
\left(\begin{array}{c}
\hat{\beta}_{1 / 4}^{+}\left(g_{-t} \gamma\right) \\
\hat{\beta}_{1 / 4}^{-}\left(g_{-t} \gamma\right)
\end{array}\right)=\exp \left(\frac{t}{2}\right)\left(\begin{array}{cc}
1 & -\frac{t}{2} \\
0 & 1
\end{array}\right)\left(\begin{array}{l}
\hat{\beta}_{1 / 4}^{+}(\gamma) \\
\hat{\beta}_{1 / 4}^{-}(\gamma)
\end{array}\right) ;
$$

(3) (Unstable horocycle invariance) For all $t \in \mathbb{R}$,

$$
\hat{\beta}_{\mu}^{ \pm}\left(h_{t}^{V} \gamma\right)=\hat{\beta}_{\mu}^{ \pm}(\gamma)
$$

(4) (Hölder property) There exists a constant $C>0$ such that, for all rectifiable arc $\gamma \subset S M$, for all $\mu \neq 1 / 4$,

$$
\left|\hat{\beta}_{\mu}^{ \pm}(\gamma)\right| \leq C\left(1+\int_{\gamma}|\hat{X}|+\int_{\gamma}|\hat{U}| \int_{\gamma}|\hat{V}|\right)\left(\int_{\gamma}|\hat{U}|\right)^{\frac{1 \mp \operatorname{Re} \nu}{2}}
$$

and, for $\mu=1 / 4(\nu=0)$,

$$
\begin{aligned}
& \left|\hat{\beta}_{1 / 4}^{+}(\gamma)\right| \leq C\left(1+\int_{\gamma}|\hat{X}|+\int_{\gamma}|\hat{U}| \int_{\gamma}|\hat{V}|\right)\left(\int_{\gamma}|\hat{U}|\right)^{\frac{1}{2}+} \\
& \left|\hat{\beta}_{1 / 4}^{-}(\gamma)\right| \leq C\left(1+\int_{\gamma}|\hat{X}|+\int_{\gamma}|\hat{U}| \int_{\gamma}|\hat{V}|\right)\left(\int_{\gamma}|\hat{U}|\right)^{\frac{1}{2}} .
\end{aligned}
$$

Notation: In the above formulas (9) and (10), the symbols $|\hat{X}|,|\hat{U}|$ and $|\hat{V}|$ stand for the transverse measures given by the forms $\hat{X}, \hat{U}$ and $\hat{V}$ respectively, and, for any $L>0$, we set

$$
L^{\frac{1}{2}+}=L^{\frac{1}{2}}(1+|\log L|) .
$$

Recall that by definition the weak unstable manifolds of the geodesic flow are the 2-dimensional manifolds tangent to the integrable distribution $\{X, V\}$ in the 
tangent bundle of $S M$. If follows immediately from the Hölder property that the restrictions of the finitely-additive measures $\hat{\beta}_{\mu}^{ \pm}$to the weak unstable manifolds of the geodesic flow vanish.

Corollary 1.1. For all Casimir parameters $\mu \in \mathbb{R}^{+}$and for any rectifiable arc $\gamma_{w u}$ contained in a (single) weak unstable manifold of the geodesic flow, we have

$$
\hat{\beta}_{\mu}^{ \pm}\left(\gamma_{w u}\right)=0 .
$$

In particular, all the finitely-additive measures $\hat{\beta}_{\mu}^{ \pm}$belong to the space $\hat{\mathfrak{B}}_{V}(S M)$.

For any $s>0$, let $\mathcal{B}_{+}^{-s}(S M)$ be the (closed) subspace of basic currents for the stable horocycle foliation supported on irreducible unitary representations of the principal and complementary series and let $\mathcal{B}_{+}^{-s}: \Omega_{1}^{-s}(S M) \rightarrow \mathcal{B}_{+}^{-s}(S M)$ be the orthogonal projection. By the Sobolev embedding theorem, for any $s>3 / 2$, any rectifiable arc $\gamma$ can be seen as a current in the dual Sobolev space $\Omega_{1}^{-s}(S M)$.

Corollary 1.2. For any $r>9 / 2$, for any $s>r+1$ and for any rectifiable arc $\gamma \subset S M$, the limit

$$
\hat{B}(\gamma):=\lim _{t \rightarrow+\infty}\left(g_{-t}^{*} \circ \mathcal{B}_{+}^{-r} \circ g_{t}^{*}\right)(\gamma) \in \Omega_{1}^{-s}(S M)
$$

exists and is equal to a uniquely determined basic current for the stable horocycle foliation. In fact, there exists a basis $\left\{B_{\mu}^{ \pm}\right\} \subset \mathcal{B}_{U}(S M)$ of eigenvectors for the action of the geodesic flow on the space of basic currents such that

$$
\hat{B}(\gamma)=\sum_{\mu \in \operatorname{Spec}(\square) \cap \mathbb{R}^{+}} \hat{\beta}_{\mu}^{+}(\gamma) B_{\mu}^{+}+\hat{\beta}_{\mu}^{-}(\gamma) B_{\mu}^{-} .
$$

For all rectifiable arcs $\gamma$ in $S M$ the following properties hold:

(1) (Additive property) For any decomposition $\gamma=\gamma_{1}+\gamma_{2}$ into subarcs,

$$
\hat{B}(\gamma)=\hat{B}\left(\gamma_{1}\right)+\hat{B}\left(\gamma_{2}\right) ;
$$

(2) (Weak unstable vanishing) For all $\gamma$ tangent to the weak unstable foliation,

$$
\hat{B}(\gamma)=0 .
$$

(3) (Unstable horocycle invariance) For all $t \in \mathbb{R}$,

$$
\hat{B}\left(h_{t}^{V} \gamma\right)=\hat{B}(\gamma)
$$

(4) (Hölder property) There exist exponents $\alpha^{ \pm} \in(0,1)$ and a constant $C>0$ such that, for all rectifiable arc $\gamma \subset S M$, we have

$$
\|\hat{B}(\gamma)\|_{-s} \leq C\left(1+\int_{\gamma}|\hat{X}|+\int_{\gamma}|\hat{U}| \int_{\gamma}|\hat{V}|\right) \max _{\alpha \in\left\{\alpha^{+}, \alpha^{-}\right\}}\left(\int_{\gamma}|\hat{U}|\right)^{\alpha}
$$

Remark 1.1. It is unclear to the authors whether the dependence of the current $\hat{B}(\gamma) \in \Omega_{1}^{\prime}(S M)$ on the rectifiable arc $\gamma \subset S M$ is continuous with respect to a natural topology (for instance the Hausdorff topology) on the space of bounded rectifiable arcs (with common endpoints). 
For any sufficiently smooth 1 -form $\lambda \in \Omega_{1}(S M)$, let $\hat{\beta}_{\lambda}$ be the finitely additive functional defined on rectifiable arcs $\gamma \subset S M$ as

$$
\hat{\beta}_{\lambda}(\gamma):=<\hat{B}(\gamma), \lambda>.
$$

It follows from Corollary 1.2 that $\hat{\beta}_{\lambda} \in \hat{\mathfrak{B}}_{V}(S M)$. In particular, for any sufficiently smooth complex-valued function $f$ on $S M$, let $\hat{\beta}_{f} \in \hat{\mathfrak{B}}_{V}(S M)$ be finitely additive functional $\hat{\beta}_{f \hat{U}}$, that is, for any rectifiable arc $\gamma \subset S M$,

$$
\hat{\beta}_{f}(\gamma)=\hat{\beta}_{f \hat{U}}(\gamma)=<\hat{B}(\gamma), f \hat{U}>.
$$

By Corollary 1.2 and the identification between basic currents and invariant distributions given by (8), the finitely-additive measure $\hat{\beta}_{f}$ has the expansion:

$$
\hat{\beta}_{f}=\sum_{\mu \in \operatorname{Spec}(\square) \cap \mathbb{R}^{+}} D_{\mu}^{+}(f) \hat{\beta}_{\mu}^{+}+D_{\mu}^{-}(f) \hat{\beta}_{\mu}^{-} .
$$

Remark 1.2. The formula (13) yields a duality between the spaces of $V$-invariant distributions and $V$-basic currents. We describe this duality in detail in $\$ \$ 1.6$

By restriction of the finitely additive measures $\hat{\beta} \in \hat{\mathfrak{B}}_{V}(S M)$ to horocycle arcs, we obtain finitely-additive Hölder cocycles $\beta$ for the stable horocycle flow $\left\{h_{t}^{U}\right\}$.

For any $(x, T) \in S M \times \mathbb{R}$ Let $\gamma_{U}(x, T)$ denote the oriented horocycle arc

$$
\gamma_{U}(x, T):=\left\{h_{t}^{U}(x) \mid t \in[0, T]\right\} .
$$

For every Casimir parameter $\mu>0$ the cocycles $\beta_{\mu}^{ \pm}$are defined as follows:

$$
\beta_{\mu}^{ \pm}(x, T):=\hat{\beta}_{\mu}^{ \pm}\left[\gamma_{U}(x, T)\right], \quad \text { for all }(x, T) \in S M \times \mathbb{R} .
$$

For any sufficiently smooth complex-valued function $f$ on $S M$, the cocycle $\beta_{f}$ is similarly defined by the formula

$$
\beta_{f}(x, T):=\hat{\beta}_{f}\left[\gamma_{U}(x, T)\right], \quad \text { for all }(x, T) \in S M \times \mathbb{R} .
$$

By construction and by formula (13), the following expansion formula holds:

$$
\beta_{f}(x, T)=\sum_{\mu \in \operatorname{Spec}(\square) \cap \mathbb{R}^{+}} D_{\mu}^{+}(f) \beta_{\mu}^{+}+D_{\mu}^{-}(f) \beta_{\mu}^{-} .
$$

Thus for every Casimir parameter $\mu \in \mathbb{R}^{+}$we obtain a pair of (linearly independent) additive Hölder cocycles $\beta_{\mu}^{ \pm}: S M \times \mathbb{R} \rightarrow \mathbb{C}$ for the stable horocycle flow. Such cocycles have the following properties.

Theorem 1.2. For any Casimir parameter $\mu \in \mathbb{R}^{+}$the following holds.

(1) (Cocycle property) For all $x \in S M$ and for all $S, T \in \mathbb{R}$ :

$$
\beta_{\mu}^{ \pm}(x, S+T)=\beta_{\mu}^{ \pm}(x, S)+\beta_{\mu}^{ \pm}\left(h_{S}^{U} x, T\right) ;
$$

(2) (Geodesic scaling) For $\mu \neq 1 / 4$, for all $x \in S M$, for all $t, T \in \mathbb{R}$,

$$
\beta_{\mu}^{ \pm}\left(g_{-t} x, T e^{t}\right)=\exp \left(\frac{1 \mp \nu}{2} t\right) \beta_{\mu}^{ \pm}(x, T),
$$


and for $\mu=1 / 4(\nu=0)$, for all $x \in S M$, for all $t, T \in \mathbb{R}$,

$$
\left(\begin{array}{c}
\beta_{1 / 4}^{+}\left(g_{-t} x, T e^{t}\right) \\
\beta_{1 / 4}^{-}\left(g_{-t} x, T e^{t}\right)
\end{array}\right)=\exp \left(\frac{t}{2}\right)\left(\begin{array}{cc}
1 & -\frac{t}{2} \\
0 & 1
\end{array}\right)\left(\begin{array}{c}
\beta_{1 / 4}^{+}(x, T) \\
\beta_{1 / 4}^{-}(x, T)
\end{array}\right) ;
$$

(3) (Hölder property) For all $\mu \neq 1 / 4$, there exists a constant $C_{\mu}>0$ such that, for all $(x, T) \in S M \times \mathbb{R}^{+}$,

$$
\left|\beta_{\mu}^{ \pm}(x, T)\right| \leq C_{\mu}|T|^{\frac{1 \mp \operatorname{Re} \nu}{2}},
$$

while for $\mu=1 / 4(\nu=0)$, there exists a constant $C>0$ such that

$$
\begin{aligned}
& \left|\beta_{1 / 4}^{+}(x, T)\right| \leq C|T|^{\frac{1}{2}+}, \\
& \left|\beta_{1 / 4}^{-}(x, T)\right| \leq C|T|^{\frac{1}{2}} .
\end{aligned}
$$

(4) (Orthogonality) For any $T \in \mathbb{R}$, the bounded function $\beta_{\mu}^{ \pm}(\cdot, T)$ belongs to the irreducible component $H_{\mu} \subset L^{2}(S M)$.

1.5. Hölder cocycles and ergodic integrals. The asymptotics and limit distributions of ergodic integrals of smooth functions is controlled by the additive Hölder cocycles for the horocycle flow introduced above. More precisely, the following approximation theorem holds.

Theorem 1.3. For any $s>11 / 2$ there exists a constant $C_{s}>0$ such that for every rectifiable curve $\gamma \subset S M$ and for all 1-forms $\lambda \in \Omega_{1}^{s}(S M)$ supported on irreducible components of the principal and complementary series, we have

$$
\left|\int_{\gamma} \lambda-\hat{B}_{\lambda}(\gamma)\right| \leq C_{s}\|\lambda\|_{s}\left(1+\int_{\gamma}|\hat{X}|+\int_{\gamma}|\hat{V}|\right) .
$$

In particular, for all functions $f \in W^{s}(S M)$ supported on irreducible components of the principal and complementary series, we have

$$
\left|\int_{\gamma} f \hat{U}-\hat{\beta}_{f}(\gamma)\right| \leq C_{s}\|f\|_{s}\left(1+\int_{\gamma}|\hat{X}|+\int_{\gamma}|\hat{V}|\right) .
$$

By the results of [12] it is possible to derive a logarithmic upper bound in the uniform norm for the the ergodic integrals along horocycle orbits of functions supported on irreducible components of the discrete series. Theorem 1.3 therefore implies the following:

Corollary 1.3. For any $s>11 / 2$ there exists a constant $C_{s}>0$ such that for all zero-average functions $f \in W^{s}(S M)$ and all $(x, T) \in S M \times \mathbb{R}^{+}$we have

$$
\left|\int_{0}^{T} f \circ h_{t}^{U}(x) d t-\beta_{f}(x, T)\right| \leq C_{s}\|f\|_{s}\left(1+\log ^{+}|T|\right) .
$$

Remark 1.3. Corollary 1.3 and the lower bounds proved in [12] on the $L^{2}$ norm of ergodic integrals imply, in particular, that the cocycles $\beta_{\mu}^{ \pm}(x, T)$ do not vanish identically as a function of $x \in S M$, for any $T \neq 0$. Indeed, to see this, it suffices to apply Corollary 1.3 to any function $f \in C^{\infty}(S M)$ such that $\beta_{f}=\beta_{\mu}^{ \pm}$. Observe 
also that, by the Ergodic Theorem, if $f$ has zero average on $S M$, then, for any fixed $T>0$, the function $\beta_{f}(x, T)$ also has zero average on $S M$.

From Corollary 1.3 we derive the following limit theorems. Recall that for any function $f \in L^{2}(S M)$ the symbol $\|f\|$ stands for its $L^{2}$-norm. For any zeroaverage real-valued function $f \in L^{2}(S M)$, for all $t>0$ and $T \in \mathbb{R}$, let $\mathfrak{M}_{t}(f)$ be the probability distribution on the real line of the random variable on $S M$ defined by the formula

$$
\mathcal{E}_{t}(f, T):=\frac{\int_{0}^{T e^{t}} f \circ h_{t}^{U}(\cdot) d t}{\left\|\int_{0}^{T e^{t}} f \circ h_{t}^{U}(\cdot) d t\right\|} .
$$

We are interested in the asymptotic behaviour (as $t \rightarrow+\infty$ ) of the probability distributions $\mathfrak{M}_{t}(f, T)$ for $T \in[0,1]$.

Let $f$ be a smooth function with non-zero orthogonal projection onto irreducible components of the complementary series. Let

$$
f=\sum_{\mu \in \operatorname{Spec}(\square)} f_{\mu}
$$

denote the decomposition of $f$ with respect to a splitting of the space $L^{2}(S M)$ into irreducible components. Let

$$
\mu_{f}:=\min \left\{\mu \in \operatorname{Spec}(\square) \backslash\{0\} \mid f_{\mu} \neq 0\right\}
$$

let $H_{1}, \ldots, H_{k} \subset L^{2}(S M)$ be all the irreducible components of Casimir parameters $\mu_{1}=\cdots=\mu_{k}=\mu_{f}$. Let $\left\{D_{1}^{ \pm}, \ldots, D_{k}^{ \pm}\right\}$denote the basis of distributional eigenvectors of the geodesic flow of the space of invariant distributions for the horocycle flow supported on $\mathcal{D}^{\prime}\left(H_{1}\right) \oplus \cdots \oplus \mathcal{D}^{\prime}\left(H_{k}\right)$. Let $\left\{\beta_{1}^{ \pm}, \ldots, \beta_{k}^{ \pm}\right\}$be the corresponding cocycles for the horocycle flow. For every $T \in \mathbb{R}$, let $P_{c p}(f, T)$ denote the probability distribution on the real line of the random variable on $S M$

$$
\frac{\sum_{i=1}^{k} D_{i}^{-}(f) \beta_{i}^{-}(\cdot, T)}{\left(\sum_{i=1}^{k}\left|D_{i}^{-}(f)\right|^{2}\left\|\beta_{i}^{-}\right\|^{2}\right)^{1 / 2}} .
$$

By Remark 1.3 and the orthogonality of cocycles, the above function is bounded, non-constant and has zero average on $S M$. The probability measure $P_{c p}(f, T)$ is therefore non-atomic and has compact support on the real line.

Let $d_{L P}$ denote the Lévy-Prohorov metric on the space of probability measures on the real line. We recall that on any separable metric space, hence, in particular, on the real line, the Lévy-Prohorov metric induces the weak* topology on the space of probability measures (see, e.g. [3]).

Theorem 1.4. There exists a constant $\alpha>0$ depending only on the surface $M$ such that the following holds. For any $s>11 / 2$ there exists a constant $C_{s}>0$ 
depending only on s such that the following holds. Let $f \in W^{s}(S M)$ be any realvalued function of zero average such that the Casimir parameter $\mu_{f} \in(0,1 / 4)$ and $\left(D_{1}^{-}(f), \ldots, D_{k}^{-}(f)\right) \neq(0, \ldots, 0)$. Let $\nu_{f}:=\sqrt{1-\mu_{f}} \in(0,1)$. Then

(1) For all $T \in[0,1]$ and all $t>0$, we have

$$
\left|\frac{\left\|\int_{0}^{T e^{t}} f \circ h_{\tau}^{U}(x) d \tau\right\|}{e^{\frac{1+\nu_{f}}{2} t}\left(\sum_{i=1}^{k}\left|D_{i}^{-}(f)\right|^{2}\left\|\beta_{i}^{-}\right\|^{2}\right)^{1 / 2}}-1\right| \leq C_{s}\|f\|_{s} e^{-\alpha t} .
$$

(2) For any $T \in[0,1]$ we have the convergence in distribution

$$
\mathfrak{M}_{t}(f, T) \rightarrow P_{c p}(f, T) \text { as } t \rightarrow \infty
$$

with the following estimate that holds for all $t>0$ uniformly in $T \in[0,1]$ :

$$
d_{L P}\left(\mathfrak{M}_{t}(f, T), P_{c p}(f, T)\right) \leq C_{s}\|f\|_{s} e^{-\alpha t} .
$$

Remark 1.4. The estimate in formula (20), uniform in $T \in \mathbb{R}$ over any compact interval, implies, in particular, that Theorem 1.4 can be strengthened to a functional limit theorem: the convergence in distribution holds in the space of measures on the space $C[0,1]$ as well, similarly to the limit theorems of [4], [5], [6].

Now we prove that, for sufficiently smooth functions supported on irreducible components of the principal series, normalized ergodic integrals converge in distribution on $S M$ to a quasi-periodic motion on an infinite-dimensional torus.

Let $\left\{\mu_{n}\right\}$ be the sequence of Casimir parameter in the interval $(1 / 4,+\infty)$ (listed with multiplicities). For all $n \in \mathbb{N}$, let $v_{n}:=\sqrt{4 \mu_{n}-1} \in \mathbb{R}^{+}$. The isotypical components of the decomposition of $L^{2}(S M)$ into irreducible representations, being eigenspaces of the Casimir operator, are closed under complex conjugation. It follows that there exists an orthogonal decomposition of $L^{2}(S M)$ into irreducible components each closed under complex conjugation. Let $\left\{D_{\mu_{n}}^{ \pm}\right\}$denote the corresponding sequence of horocycle invariant distributions, and let $\left\{\beta_{\mu_{n}}^{ \pm}\right\}$the sequence of additive Hölder cocycles described in Theorem 1.2 By the characterization of the distributions $\left\{D_{\mu_{n}}^{ \pm}\right\}$as distributional eigenvectors of the geodesic flow and by the construction of the cocycles $\left\{\beta_{\mu_{n}}^{ \pm}\right\}$, it follows that, for all $n \in \mathbb{N}$,

$$
D_{\mu_{n}}^{-}=\overline{D_{\mu_{n}}^{+}} \quad \text { and } \quad \beta_{\mu_{n}}^{-}=\overline{\beta_{\mu_{n}}^{+}} \text {. }
$$

For any $s>11 / 2$, let $f \in W^{s}(S M)$ be a real-valued function supported on irreducible components of the principal series. By definition and by formula (21), the cocycle $\beta_{f}: S M \times \mathbb{R} \rightarrow \mathbb{C}$ is also real-valued, and from (13) we have

$$
\beta_{f}(x, T)=\operatorname{Re}\left[\sum_{n \in \mathbb{N}} D_{\mu_{n}}^{+}(f) \beta_{\mu_{n}}^{+}(x, T)\right], \quad \text { for all }(x, T) \in S M \times \mathbb{R} .
$$

Let $\mathbb{T}^{\infty}:=(\mathbb{R} / 2 \pi \mathbb{Z})^{\infty}$ be the infinite-dimensional torus endowed with the product topology. For any real-valued function $f \in W^{s}(S M)$ supported on irreducible unitary components of the principal series and for all $\theta \in \mathbb{T}^{\infty}$, let

$$
\beta(f, \theta, x, T):=\operatorname{Re}\left[\sum_{n \in \mathbb{N}} D_{\mu_{n}}^{+}(f) e^{i \theta_{n}} \beta_{\mu_{n}}^{+}(x, T)\right], \quad \text { for all }(x, T) \in S M \times \mathbb{R} .
$$


For $\theta \in \mathbb{T}^{\infty}$ and $T>0$, let $P_{p r}(f, \theta, T)$ be the probability distribution of the random variable given by the formula

$$
\frac{\beta(f, \theta, \cdot, T)}{\|\beta(f, \theta, \cdot, T)\|}, \quad \text { for all } x \in S M
$$

Since the random variables $\beta(f, \theta, \cdot, T)$ on $S M$ are non-constant and bounded, the probability distributions $P(f, \theta, T)$ are non-atomic compactly supported measures on the real line (uniformly with respect to $T \in[0,1]$ ).

Our main result on the asymptotics of distributions of normalized ergodic integrals for real-valued functions supported on the principal series is the following

Theorem 1.5. For any $s>11 / 2$ there exists a constant $C_{s}>0$ such that the following holds for any real-valued function $f \in W^{s}(S M)$ supported on the irreducible components of the principal series such that $\left\{D_{\mu_{n}}^{+}(f)\right\} \neq 0$ in $\ell^{1}(\mathbb{N}, \mathbb{C})$.

(1) For all $T \in[0,1]$ and all $t>0$, we have

$$
\left|\frac{\left\|\int_{0}^{T e^{t}} f \circ h_{\tau}^{U}(x) d \tau\right\|}{e^{\frac{t}{2}}\left\|\beta\left(f, \frac{v t}{2}, \cdot, T\right)\right\|}-1\right| \leq C_{s}\|f\|_{s} e^{-\frac{t}{2}} .
$$

(2) For all $T \in[0,1]$ and all $t>0$, we have

$$
d_{L P}\left(\varepsilon_{t}(f, T), P_{p r}\left(f, \frac{v t}{2}, T\right)\right) \leq C_{s}\|f\|_{s} e^{-\frac{t}{2}} .
$$

The above theorem implies that for real-valued functions supported on the principal series limit distributions exist along sequence of time such that the orbit of the toral translation of frequency $v / 2 \in \mathbb{R}^{\infty}$ on the infinite torus $\mathbb{T}^{\infty}$ converges. We conjecture that the limit does not exist otherwise. Below from Theorem 1.5 we derive some restrictions on limit distributions.

Definition 1.2. Let $H \subset L^{2}(S M)$ be a $P S L(2, \mathbb{R})$-invariant subspace which is a direct sum of finitely many irreducible components of the principal series, that is,

$$
H=\sum_{i=1}^{n} H_{i}
$$

The subspace $H$ is called Casimir simple if all the corresponding Casimir parameters $\left\{\nu_{1}, \ldots, \nu_{n}\right\}$ are distinct. The subspace $H$ is called Casimir irrational if the Casimir parameters $\left\{\nu_{1}, \ldots, \nu_{n}\right\}$ are rationally independent.

We derive the following conditional uniqueness result for the principal series.

Corollary 1.4. Let $H \subset L^{2}(S M)$ be any Casimir simple $P S L(2, \mathbb{R})$-invariant subspace. If the limit distribution of the family of random variables

$$
\frac{\int_{0}^{T} f \circ h_{t}^{U} d t}{\left\|\int_{0}^{T} f \circ h_{t}^{U} d t\right\|}
$$

exists for any given $f \in C^{\infty}(H)$ which is not a coboundary, then the limit distribution is unique in the sense that it does not depend on the function. 
Finally, we derive restrictions on the joint probability distribution of the cocycle functions in case limit distributions exist for all function supported on a Casimir irrational subspace.

For any irreducible representation $H_{\mu} \subset L^{2}(S M)$ of the principal series, we have constructed Hölder cocycle functions $\beta:=\beta_{\mu}^{+}$and $\bar{\beta}=\beta_{\mu}^{-}: S M \rightarrow \mathbb{C}$. For any $P S L(2, \mathbb{R})$-invariant subspace $H \subset L^{2}(S M)$ supported on finitely many irreducible components of the principal series, let

$$
\beta_{H}:=\left(\beta_{1}, \ldots, \beta_{n}\right): S M \rightarrow \mathbb{C}^{n}
$$

be the corresponding vector-valued cocycle function.

Given any function $\beta: S M \rightarrow \mathbb{C}$, let $T_{\beta}: \mathbb{C} \rightarrow \mathbb{C}$ be the affine transformation defined as follows. Let $R_{\beta}$ be the rotation by the angle $\theta_{\beta} \in[0,2 \pi)$ such that

$$
e^{2 i \theta_{\beta}} \int_{S M} \beta^{2} d \mathrm{vol} \in \mathbb{R}^{+} \cup\{0\} .
$$

For any pair $(A, B)$ of positive real numbers, let $T_{A, B}: \mathbb{C} \rightarrow \mathbb{C}$ the affine map

$$
T_{A, B}(x, y):=(x / A, y / B), \quad \text { for all }(x, y) \in \mathbb{R}^{2} \equiv \mathbb{C} .
$$

Let $\left(A_{\beta}, B_{\beta}\right)$ be the positive real numbers given by the formulas:

$$
\left\{\begin{array}{l}
A_{\beta}^{2}=\left(\|\beta\|^{2}+\left|\int_{S M} \beta^{2} \omega\right|\right) / 2, \\
B_{\beta}^{2}=\left(\|\beta\|^{2}-\left|\int_{S M} \beta^{2} \omega\right|\right) / 2 .
\end{array}\right.
$$

It is proved in Section 5.3 that $\int_{S M} \beta^{2} \omega \neq 0$, hence $A_{\beta}^{2}>B_{\beta}^{2}$. Let then $T_{\beta}$ be given by the formula

$$
T_{\beta}:=T_{A_{\beta}, B_{\beta}} \circ R_{\beta} .
$$

Given any function $\beta:=\left(\beta_{1}, \ldots, \beta_{n}\right) \rightarrow \mathbb{C}^{n}$, let $T_{\beta}$ be the product affine map

$$
T_{\beta}=T_{\beta_{1}} \times \ldots T_{\beta_{n}}: \mathbb{C}^{n} \rightarrow \mathbb{C}^{n} .
$$

Corollary 1.5. Let $H \subset L^{2}(S M)$ be any Casimir irrational $P S L(2, \mathbb{R})$-invariant subspace. The limit distribution of the family of random variables

$$
\frac{\int_{0}^{T} f \circ h_{t}^{U} d t}{\left\|\int_{0}^{T} f \circ h_{t}^{U} d t\right\|}
$$

exists for all $f \in C^{\infty}(H)$ which is not a coboundary if and only if the function $T_{\beta_{H}} \circ \beta_{H}: S M \rightarrow \mathbb{C}^{n}$ has a rotationally invariant probability distribution.

1.6. Duality theorems. The formalism of finitely-aditive measures allows us to establish a duality between the spaces of distributions invariant under the stable and the unstable horocycle flows, respectively; more precisely, between the subspaces of invariant distributions corresponding to the positive eigenvalues of the Casimir operator.

Finitely additive $V$-invariant 1-dimensional Hölder measures on rectifiable arcs induce by integration currents of dimension 2 (and degree 1 ). In fact, let $\hat{\beta} \in$ 
$\hat{\mathfrak{B}}_{V}(S M)$. Given a smooth 2 -form $\eta$, using the Hölder property and the (finite) additivity of $\hat{\beta}$, one can define the integral

$$
\int_{S M} \hat{\beta} \otimes \eta
$$

as the limit of Riemann sums. The correspondence

$$
\eta \rightarrow \int_{S M} \hat{\beta} \otimes \eta
$$

now yields a current on $S M$ of dimension 2 (and degree 1 ), which, slightly abusing notation, we denote by the same symbol $\hat{\beta}$. The current $\hat{\beta}$ defined above in fact extends to continuous forms and, by the Sobolev embedding theorem, to forms in Sobolev spaces.

Our next aim is to describe the currents $\hat{\beta}_{\mu}^{ \pm}$in terms of distributions invariant under the unstable horocycle flow $\left\{h_{t}^{V}\right\}$.

Given a Casimir parameter $\mu>0$, consider the finitely-additive measure

$$
\hat{D}_{\mu}^{ \pm}=\hat{X} \otimes \hat{\beta}_{\mu}^{ \pm} \otimes \hat{V}
$$

Since, for any $f \in C^{\infty}(S M)$, the integral of $f$ with respect to the measure $\hat{D}_{\mu}^{ \pm}$ can be defined as the limit of Riemann sums, the measure $\hat{D}_{\mu}^{ \pm}$yields a distribution (in the sense of S.L. Sobolev and L. Schwartz) on $C^{\infty}(S M)$; slightly abusing notation, we denote the distribution by the same symbol $\hat{D}_{\mu}^{ \pm}$.

Theorem 1.6. For every Casimir parameter $\mu \in \mathbb{R}^{+}$, the distributions $\hat{D}_{\mu}^{ \pm}$given by (24) are $V$-invariant. For $\mu \neq 1 / 4$, they satisfy the identities

$$
\mathcal{L}_{X} \hat{D}_{\mu}^{ \pm}=\frac{1 \pm \nu}{2} \hat{D}_{\mu}^{ \pm}
$$

while for $\mu=1 / 4(\nu=0)$, they satisfy the identity

$$
\mathcal{L}_{X}\left(\begin{array}{l}
\hat{D}_{1 / 4}^{+} \\
\hat{D}_{1 / 4}^{-}
\end{array}\right)=\frac{1}{2}\left(\begin{array}{ll}
1 & 1 \\
0 & 1
\end{array}\right)\left(\begin{array}{l}
\hat{D}_{1 / 4}^{+} \\
\hat{D}_{1 / 4}^{-}
\end{array}\right) .
$$

Theorem 1.6 can be equivalently reformulated as follows.

Given a distribution $D$ acting on $C^{\infty}(S M)$, let $D \wedge \hat{U}$ denote the current of degree 1 (and dimension 2) defined as the exterior product of the the distribution $D$, identified to a current of degree 0 (and dimension 3 ) via the normalized volume form $\omega$, times the smooth 1 -form $\hat{U}$ on $S M$; that is, the current given by the following formula: for any smooth 2-form $\eta$ on $S M$,

$$
(D \wedge \hat{U})(\eta):=D\left(\frac{\hat{U} \wedge \eta}{\omega}\right) .
$$

Theorem 1.7. For every Casimir parameter $\mu \in \mathbb{R}^{+}$, there exist $V$-invariant distributions $\hat{D}_{\mu}^{ \pm} \in \mathcal{D}^{\prime}\left(H_{\mu}\right)$ such that

$$
\hat{\beta}_{\mu}^{ \pm}=\hat{D}_{\mu}^{ \pm} \wedge \hat{U}
$$


For all $\mu \neq 1 / 4$, the distributions $\hat{D}_{\mu}^{ \pm}$are eigenvectors of the geodesic flow, that is, they satisfy the identitities

$$
\mathcal{L}_{X} \hat{D}_{\mu}^{ \pm}=\frac{1 \pm \nu}{2} \hat{D}_{\mu}^{ \pm}
$$

while for $\mu=1 / 4(\nu=0)$ they are generalized eigenvectors, that is,

$$
\mathcal{L}_{X}\left(\begin{array}{l}
\hat{D}_{1 / 4}^{+} \\
\hat{D}_{1 / 4}^{-}
\end{array}\right)=\frac{1}{2}\left(\begin{array}{ll}
1 & 1 \\
0 & 1
\end{array}\right)\left(\begin{array}{l}
\hat{D}_{1 / 4}^{+} \\
\hat{D}_{1 / 4}^{-}
\end{array}\right) .
$$

The duality theorem (Theorem 1.7) leads to the classification theorem stated below. Let $\hat{\mathfrak{B}}_{V}(S M)$ be the space of all finitely additive 1-dimensional Hölder measures introduced in Definition 1.1 For any $s>0$, let $\Omega_{2}^{-s}(S M)$ be the Sobolev space of currents of dimension 2 (and degree 1) defined as the dual space of the Sobolev space $\Omega_{2}^{s}(S M)$ of 2 -forms on $S M$. By the Sobolev embedding theorem, the space $\hat{\mathfrak{B}}_{V}(S M)$ embeds as closed subspace, denoted as $\hat{\mathfrak{B}}_{V}^{-s}(S M)$, into $\Omega_{2}^{-s}(S M)$.

Theorem 1.8. For all $s>3 / 2$, the Hilbert space $\hat{\mathfrak{B}}_{V}^{-s}(S M)$ is spanned by the system of finitely-additive measures $\left\{\hat{\beta}_{\mu}^{ \pm} \mid \mu \in \operatorname{Spec}(\square) \cap \mathbb{R}^{+}\right\}$.

The duality theorem (Theorem 1.7) also leads to a direct bijective correspondence between the lift of the finitely-additive measures $\hat{\beta}_{\mu}^{ \pm}$to $P S L(2, \mathbb{R})$ (denoted below by the same symbol) and the $\Gamma$-invariant conformal distributions on the boundary of the Poincaré disk studied by S. Cosentino in [9].

Theorem 1.9. For any Casimir parameter $\mu \in \mathbb{R}^{+} \backslash\{1 / 4\}$, there exist on the boundary of the Poincaré disk $\Gamma$-invariant conformal distributions $\phi_{\mu}^{ \pm}$of exponents $(1 \mp \nu) / 2$ such that the following identities hold on $\operatorname{PSL}(2, \mathbb{R})$ :

$$
\hat{\beta}_{\mu}^{ \pm} \otimes d t=\phi_{\mu}^{ \pm} \otimes e^{-\left(\frac{1 \mp \nu}{2}\right) t} d t .
$$

For $\mu=1 / 4(\nu=0)$, on the boundary of the Poincaré disk there exist a $\Gamma$-invariant conformal distribution $\phi_{1 / 4}$ of exponent $1 / 2$ and a distribution $\phi_{1 / 4}^{\prime}$ of order $1 / 2^{+}$ (in the Hölder sense) such that

$$
\begin{aligned}
& \hat{\beta}_{1 / 4}^{+} \otimes d t=\phi_{1 / 4}^{\prime} \otimes e^{-\frac{t}{2}} d t+\phi_{1 / 4} \otimes \frac{t}{2} e^{-\frac{t}{2}} d t, \\
& \hat{\beta}_{1 / 4}^{-} \otimes d t=\phi_{1 / 4} \otimes e^{-\frac{t}{2}} d t .
\end{aligned}
$$

Remark 1.5. For $\mu=1 / 4$, the space of all $\Gamma$-invariant conformal distributions of exponent $1 / 2$ is 1 -dimensional in each irreducible component.

1.7. Organization of the paper. The paper is organized as follows. In Section 2 we construct finitely additive measures of rectifiable arcs and prove our main results about them (in particular Theorem 1.1, Corollary 1.1 and Corollary 1.2 up to a technical estimate (Lemma 2.4) which will be proved in $\$ \S 5.2$. In Section 3 we prove our results on additive cocycles for the horocycle flow (Theorem 1.2) and the approximation theorem for ergodic integrals (Theorem 1.3). From the approximation theorem, we then derive our results on limit distributions (Theorem 1.4. 
Theorem 1.5, Corollary 1.4 and Corollary 1.5 . Section 4 is devoted to the proof of the duality theorem (Theorem 1.7), of the classification theorem (Theorem 1.8) and to the relations with $\Gamma$-invariant conformal distributions (Theorem 1.9). In Section 5 we collect several technical auxiliary results. In $\S \S 5.2$ we prove the abovementioned estimate we need in the construction of finitely additive measures and additive cocycles (Lemma 2.4). In $\S \S 5.3$ and $\S \S 5.4$ we prove the technical lemmas needed in the proof of our conditional theorems on existence of limit distributions for functions supported on irreducible components of the principal series.

1.8. Acknowledgements. . We are deeply grateful to Yakov Sinai for suggesting the problem to us and for his friendly encouragement. We are deeply grateful to Artur Avila for pointing out the orthogonality property of additive cocycles stated in Theorem 1.2 .

A.I. B. is an Alfred P. Sloan Research Fellow. During work on this project, he was supported in part by Grant MK-4893.2010.1 of the President of the Russian Federation, by the Programme on Mathematical Control Theory of the Presidium of the Russian Academy of Sciences, by the Programme 2.1.1/5328 of the Russian Ministry of Education and Research, by the Edgar Odell Lovett Fund at Rice University, by the National Science Foundation under grant DMS 0604386, and by the RFBR-CNRS grant 10-01-93115.

G.F. was supported by the National Science Foundation grant DMS 0800673.

2. Basic Currents and Finitely-Additive Measures on Rectifiable ARCS.

In this section we prove Theorem 1.1 up to a technical estimate which will be proved in the $\S \S 5.2$. We then derive Corollary 1.1 and Corollary 1.2 .

2.1. Basic currents. By definition, the volume form $\omega$ on $S M$ can be written as

$$
\omega=\hat{X} \wedge \hat{U} \wedge \hat{V} \text {. }
$$

The contractions $\eta_{X}:=\imath_{X} \omega, \eta_{U}:=\imath_{U} \omega$ and $\eta_{V}:=\imath_{V} \omega$ are closed 2-forms which can be written as follows:

$$
\eta_{X}=\hat{U} \wedge \hat{V}, \quad \eta_{U}=-\hat{X} \wedge \hat{V}, \quad \eta_{V}=\hat{X} \wedge \hat{U} .
$$

We recall that a distribution $D \in \mathcal{D}^{\prime}(S M)$ (in the sense of S.L. Sobolev and L. Schwartz) is called $U$-invariant (or invariant under the stable horocycle flow $\left\{h_{t}^{U}\right\}$ ) iff $\mathcal{L}_{U} D=0$ in $\mathcal{D}^{\prime}(S M)$. A current $C$ of degree 2 (and dimension 1) is called basic for the orbit foliation of the stable horocycle flow if and only if

$$
\mathcal{L}_{U} C=\imath_{U} C=0 \text { in } \mathcal{D}^{\prime}(S M) .
$$

(The operators $\mathcal{L}_{U}$ and $\iota_{U}$ are respectively the Lie derivative and the contraction with respect to the horocycle generator $U$ acting on currents according to the standard definition).

Let $\mathcal{J}_{U}(S M)$ denote the space of all $U$-invariant distributions and $\mathcal{B}_{U}(S M)$ denote the space of all basic currents of degree 2 (and dimension 1) for the orbit foliation of the stable horocycle flow. 
For every $s \geq 0$, let $W^{s}(S M)$ be the standard Sobolev space on the compact manifolds $S M$ and let $\Omega_{1}^{s}(S M)$ be the Sobolev space of all 1-forms on $S M$ defined as follows:

$$
\lambda:=\lambda_{X} \hat{X}+\lambda_{U} \hat{U}+\lambda_{V} \hat{V} \in \Omega_{1}^{s}(S M) \Leftrightarrow\left(\lambda_{X}, \lambda_{U}, \lambda_{V}\right) \in\left[W^{s}(S M)\right]^{3} .
$$

Let $W^{-s}(S M)$ and $\Omega_{1}^{-s}(S M)$ denote the Sobolev spaces dual of the (Hilbert) spaces $W^{s}(S M)$ and $\Omega_{1}^{s}(S M)$ respectively. The space $W^{-s}(S M)$ which can be viewed either as currents of degree 3 and dimension 0 (linear functionals on functions) or as currents of degree 0 and dimension 3 (linear functional on 3forms). A standard $S L(2, \mathbb{R})$-invariant identification between functions and 3forms is in fact given by the volume form on $S M$. The space $\Omega_{1}^{-s}(S M)$ is a space of currents of degree 2 and dimension 1 (linear functionals on 1-forms). Let $\mathcal{B}_{U}^{-s}(S M) \subset \Omega_{1}^{-s}(S M)$ denote the subspace of basic currents for the orbit foliation of the stable horocycle flow, that is, of currents satisfying the identities (26). It is a standard fact, easy to prove, that the space $\mathcal{B}_{U}^{-s}(S M)$ of basic currents is isomorphic to the space $\mathcal{J}_{U}^{-s}(S M)$ of $U$-invariant distributions:

Lemma 2.1. For any $s \in \mathbb{R}$ the correspondence $D \rightarrow D \eta_{U}$ defines an isomorphism from the space $\mathcal{J}_{U}^{-s}(S M)$ of invariant distributions for the stable horocycle flow onto the space $\mathcal{B}_{U}^{-s}(S M)$ of basic currents for its orbit foliation.

Proof. Let $D$ be any $U$-invariant distribution. It follows that $C:=D \eta_{U}$ is closed. By definition $\imath_{U} C=0$. It follows that

$$
\mathcal{L}_{U} C=\imath_{U} d C+d \imath_{U} C=0,
$$

hence $C$ is a basic current for the stable horocycle foliation. Conversely, let $C$ be any basic current for the stable horocycle foliation and let $D:=C \wedge \hat{U}$. We claim that $D$ is $U$-invariant. Since $\mathcal{L}_{U} C=\imath_{U} C=0$, a computation yields

$$
\mathcal{L}_{U} D=\mathcal{L}_{U} C \wedge \hat{U}+C \wedge \mathcal{L}_{U} \hat{U}=C \wedge \imath_{U}(d \hat{U})=\imath_{U} C \wedge d \hat{U}=0 .
$$

Finally, since $\imath_{U} C=0$, it follows that

$$
D \eta_{U} \equiv \iota_{U} D=\imath_{U}(C \wedge \hat{U})=C,
$$

hence the map $D \rightarrow D \eta_{U}$ is a bijection of the space of all invariant distributions onto the space of all basic currents with inverse given by the map $C \rightarrow C \wedge \hat{U}$. It follows from the definition of the Sobolev spaces of currents that the above maps are isomorphisms between the dual Sobolev spaces $\mathcal{J}_{U}^{-s}(S M)$ and $\mathcal{B}_{U}^{-s}(S M)$.

2.2. Geodesic scaling of basic currents. Let $H_{\mu} \subset L^{2}(S M)$ be any non-trivial irreducible component with Casimir parameter $\mu \in \mathbb{R} \backslash\{0\}$. Let $W^{-s}\left(H_{\mu}\right)$ and $\Omega_{1}^{-s}\left(H_{\mu}\right)$ denote the associated Sobolev spaces of distributions and, respectively, currents of dimension 1 (and degree 2). The subspaces $W^{-s}\left(H_{\mu}\right)$ and $\Omega_{1}^{-s}\left(H_{\mu}\right)$ are $S L(2, \mathbb{R})$-invariant irreducible components of the decomposition of the dual Sobolev spaces $W^{-s}(S M)$ and $\Omega_{1}^{-s}(S M)$ respectively. Let $\mathcal{B}_{U}^{-s}\left(H_{\mu}\right)$ denote the associated $S L(2, \mathbb{R})$-invariant irreducible component of the space $\mathcal{B}_{U}^{-s}(S M)$ of basic currents for the stable horocycle foliation, that is, for all $\mu \in \mathbb{R} \backslash\{0\}$,

$$
\mathcal{B}^{-s}\left(H_{\mu}\right):=\mathcal{B}^{-s}(S M) \cap \Omega_{1}^{-s}\left(H_{\mu}\right) .
$$


The following result describes the (infinitesimal) action of the geodesic flow on the space $\mathcal{B}_{U}^{-s}\left(H_{\mu}\right)$ of basic currents for all Casimir parameters $\mu \in \mathbb{R} \backslash\{0\}$.

Lemma 2.2. For any $s>1$ and $\mu \in \mathbb{R}^{+} \backslash\{1 / 4\}$, the space $\mathcal{B}_{U}^{-s}\left(H_{\mu}\right)$ has complex dimension 2 and has a basis $\left\{B_{\mu}^{+}, B_{\mu}^{-}\right\}$of eigenvectors for the action of the geodesic flow. In fact, the following formulas hold:

$$
\mathcal{L}_{X} B_{\mu}^{ \pm}=\frac{1 \mp \nu}{2} B_{\mu}^{ \pm}
$$

For $\mu=1 / 4(\nu=0)$, the space $\mathcal{B}_{U}^{-s}\left(H_{\mu}\right)$ has complex dimension 2 and has a basis $\left\{B_{\mu}^{+}, B_{\mu}^{-}\right\}$of generalized eigenvectors for the action of the geodesic flow. The following formula holds:

$$
\mathcal{L}_{X}\left(\begin{array}{l}
B_{1 / 4}^{+} \\
B_{1 / 4}^{-}
\end{array}\right)=\frac{1}{2}\left(\begin{array}{cc}
1 & 0 \\
-1 & 1
\end{array}\right)\left(\begin{array}{l}
B_{1 / 4}^{+} \\
B_{1 / 4}^{-}
\end{array}\right) .
$$

For $\mu=-n^{2}+n<0(\nu=2 n-1)$ and $s>n$, the space $\mathcal{B}_{U}^{-s}\left(H_{\mu}\right)$ has complex dimension 1 and has a basis $\left\{B_{\mu}\right\}$, containing a single eigenvector for the action of the geodesic flow. In fact, the following formula holds:

$$
\mathcal{L}_{X} B_{\mu}=\frac{1-\nu}{2} B_{\mu}=(1-n) B_{\mu} .
$$

Proof. It follows by Lemma2.1 that the space $\mathcal{B}_{U}^{-s}\left(H_{\mu}\right)$ is isomorphic to the space $\mathcal{J}_{U}^{-s}\left(H_{\mu}\right):=\mathcal{J}_{U}(S M) \cap W^{-s}\left(H_{\mu}\right)$ of invariant distributions. By [12], Theorem 3.2, for any $\mu \in \mathbb{R}^{+} \backslash\{1 / 4\}$, the space $\mathcal{J}_{U}^{-s}\left(H_{\mu}\right)$ has complex dimension 2 and has a basis $\left\{D_{\mu}^{+}, D_{\mu}^{-}\right\}$of eigenvectors of the geodesic flow, in the sense that the following formulas hold:

$$
\mathcal{L}_{X} D_{\mu}^{ \pm}=-\frac{1 \pm \nu}{2} D_{\mu}^{ \pm}
$$

Let $B_{\mu}^{ \pm}:=D_{\mu}^{ \pm} \eta_{U}$. Since $U$ is the generator of the stable horocycle flow, we have the following equality of currents:

$$
\mathcal{L}_{X} \eta_{U}=\eta_{U}
$$

The statement for the case $\mu \neq 1 / 4$ then follows since

$$
\mathcal{L}_{X} B_{\mu}^{ \pm}=\left(\mathcal{L}_{X} D_{\mu}^{ \pm}+D_{\mu}^{ \pm}\right) \eta_{U}=\frac{1 \mp \nu}{2} B_{\mu}^{ \pm} .
$$

In the case $\mu=1 / 4$, by [12], Lemma 3.5, the space $\mathcal{J}_{U}^{-s}\left(H_{\mu}\right)$ has complex dimension 2 and has a basis $\left\{D_{\mu}^{+}, D_{\mu}^{-}\right\}$of generalized eigenvectors of the geodesic flow, in the sense that the following formulas hold:

$$
\mathcal{L}_{X}\left(\begin{array}{c}
D_{\mu}^{+} \\
D_{\mu}^{-}
\end{array}\right)=-\frac{1}{2}\left(\begin{array}{cc}
1 & 0 \\
1 & 1
\end{array}\right)\left(\begin{array}{c}
D_{\mu}^{+} \\
D_{\mu}^{-}
\end{array}\right) .
$$

Let $B_{\mu}^{ \pm}:=D_{\mu}^{ \pm} \eta_{U}$. Formula (28) then follows by Leibniz rule from the above formula and formula (30). In fact,

$$
\mathcal{L}_{X}\left(\begin{array}{c}
B_{\mu}^{+} \\
B_{\mu}^{-}
\end{array}\right)=\left[-\frac{1}{2}\left(\begin{array}{ll}
1 & 0 \\
1 & 1
\end{array}\right)+\left(\begin{array}{ll}
1 & 0 \\
0 & 1
\end{array}\right)\right]\left(\begin{array}{c}
B_{\mu}^{+} \\
B_{\mu}^{-}
\end{array}\right)
$$


Similarly, formula (29) follows from [12], Lemma 3.5. In fact, for any Casimir parameter $\mu=-n^{2}+n<0$, the space $\mathcal{J}_{U}^{-s}\left(H_{\mu}\right)$ has complex dimension 1 and has a basis $\left\{D_{\mu}\right\}$ containing a single eigenvector of the geodesic flow such that

$$
\mathcal{L}_{X} D_{\mu}=-\frac{1+\nu}{2} D_{\mu}=-n D_{\mu}
$$

$>$ From the above formula it follows that

$$
\mathcal{L}_{X} B_{\mu}=\left(\mathcal{L}_{X} D_{\mu}+D_{\mu}\right) \eta_{U}=\frac{1-\nu}{2} B_{\mu}=(1-n) B_{\mu} .
$$

The Lemma is proved.

2.3. Orthogonal projections on basic currents. For any $s>1 / 2$ we have the orthogonal direct sum decomposition

$$
\Omega_{1}^{-s}(S M)=\mathcal{B}_{U}^{-s}(S M) \oplus^{\perp} \mathcal{B}_{U}^{-s}(S M)^{\perp}
$$

Let $\mathcal{B}^{-s}: \Omega_{1}^{-s}(S M) \rightarrow \mathcal{B}_{U}^{-s}(S M)$ denote the orthogonal projection onto the subspace of basic currents and $\mathcal{R}^{-s}: \Omega_{1}^{-s}(S M) \rightarrow \mathcal{B}_{U}^{-s}(S M)^{\perp}$ denote the orthogonal projection onto its orthogonal complement .

Let $\Pi_{\mu}^{-s}: \Omega_{1}^{-s}(S M) \rightarrow \Omega_{1}^{-s}\left(H_{\mu}\right)$ be the orthogonal projection. We remark that the projections $\Pi_{\mu}^{-s}$ commute with the action of $S L(2, \mathbb{R})$, hence in particular with the action of the geodesic flow, on the Sobolev space $\Omega_{1}^{-s}(S M)$.

Let $\mathcal{B}_{\mu}^{-s}:=\Pi_{\mu}^{-s} \circ \mathcal{B}^{-s}=\mathcal{B}^{-s} \circ \Pi_{\mu}^{-s}$ be the orthogonal projection onto the subspace $\mathcal{B}_{U}^{-s}\left(H_{\mu}\right) \subset \Omega_{1}^{-s}\left(H_{\mu}\right)$. Let $\mathcal{R}_{\mu}^{-s}:=\Pi_{\mu}^{-s} \circ \mathcal{R}^{-s}=\mathcal{R}^{-s} \circ \Pi_{\mu}^{-s}$ be the complementary projection on the space $\mathcal{B}_{U}^{-s}(S M)^{\perp} \cap \Omega_{1}^{-s}\left(H_{\mu}\right)$. We remark that the projections $\mathcal{B}_{\mu}^{-s}$ and $\mathcal{R}_{\mu}^{-s}$ do not necessarily commute with the action of the geodesic flow. However, the range of the projection $\mathcal{B}_{\mu}^{-s}$, which is the space $\mathcal{B}_{U}^{-s}\left(H_{\mu}\right)$ of basic currents, is invariant under the action of the geodesic flow.

By the Sobolev embedding theorem, any rectifiable arc $\gamma$ can be viewed as a current of dimension 1 (and degree 2) in $\Omega_{1}^{-s}(S M)$ for any $s>3 / 2$. For all non-trivial irreducible unitary representations of Casimir parameter $\mu \in \mathbb{R}$, let $\mathcal{B}_{\mu}^{-s}(\gamma) \in \Omega_{1}^{-s}\left(H_{\mu}\right)$ denote the projection $\Pi_{\mu}^{-s} \circ \mathcal{B}^{-s}(\gamma)$ of the current $\mathcal{B}_{\mu}^{-s}(\gamma)$ onto the irreducible subspace $\Omega_{1}^{-s}\left(H_{\mu}\right) \subset \Omega_{1}^{-s}(S M)$. We then write

$$
\mathcal{B}_{\mu}^{-s}(\gamma):= \begin{cases}\hat{\alpha}_{\mu,-s}^{+}(\gamma) B_{\mu}^{+}+\hat{\alpha}_{\mu,-s}^{-}(\gamma) B_{\mu}^{-}, & \text {for } \mu \in \operatorname{Spec}(\square) \cap \mathbb{R}^{+} \\ \hat{\alpha}_{\mu,-s}(\gamma) B_{\mu}, & \text { for } \mu \in \operatorname{Spec}(\square) \cap \mathbb{R}^{-}\end{cases}
$$

In other words, the complex numbers $\hat{\alpha}_{\mu,-s}^{ \pm}(\gamma), \hat{\alpha}_{\mu}(\gamma)$ are the components of the current $\gamma$ in the direction of the basic currents $B_{\mu}^{ \pm}, B_{\mu}$ (that is, by definition, the coefficients of the currents $B_{\mu}^{ \pm}, B_{\mu}$ in the orthogonal projection of the current $\gamma$ onto the closed subspace of all basic currents).

We recall that the subspace $\mathcal{B}_{U}^{-s}\left(H_{\mu}\right) \subset \Omega_{1}^{-s}\left(H_{\mu}\right)$ is trivial for all Casimir parameters $\mu=-n^{2}+n$ (discrete series) whenever $s \leq n \in \mathbb{Z}^{+}$. In this case the component $\hat{\alpha}_{\mu,-s}$ is defined as zero. 
Lemma 2.3. For every $\mu_{0}>1 / 4$ and for every $s>1 / 2$ the system $\left\{B_{\mu}^{+}, B_{\mu}^{-}\right\}$has uniformly bounded distorsion in $\Omega_{1}^{-s}(S M)$ for all Casimir parameters $\mu \geq \mu_{0}$, that is, there exists a constant $C_{s}\left(\mu_{0}\right)>0$ such that, for all $\mu \geq \mu_{0}$,

$$
\sup _{\left(\alpha^{+}, \alpha^{-}\right) \in \mathbb{R}^{2} \backslash\{0\}} \frac{\left\|\alpha^{+} B_{\mu}^{+}\right\|_{-s}+\left\|\alpha^{-} B_{\mu}^{-}\right\|_{-s}}{\left\|\alpha^{+} B_{\mu}^{+}+\alpha^{-} B_{\mu}^{-}\right\|_{-s}} \leq C_{s}\left(\mu_{0}\right) .
$$

Proof. As observed in the proof of Lemma 5.1 in [12], if the Casimir parameter $\mu \geq \mu_{0}>1 / 4$, for any $s>1 / 2$, the distorsion in $W^{-s}(S M)$ of the system of distributions $\left\{D_{\mu}^{+}, D_{\mu}^{-}\right\}$stays uniformly bounded above (in other terms, the angle in $W^{-s}(S M)$ between $D_{\mu}^{+}$and $D_{\mu}^{-}$stays uniformly bounded below), and in fact this bound is also uniform with respect to $s>1$. By Lemma 2.1, the map $D \rightarrow D \eta_{U}$ defines an isomorphism from the space $\mathcal{J}_{U}^{-s}(S M)$ of invariant distribution onto the space $\mathcal{B}_{U}^{-s}(S M)$ of basic currents. It follows that for all Casimir parameters $\mu>0$ the distorsion in $\Omega_{1}^{-s}(S M)$ of the system of basic current $\left\{B_{\mu}^{+}, B_{\mu}^{-}\right\}$is equal to the distorsion in $W^{-s}(S M)$ of the system of invariant distributions $\left\{D_{\mu}^{+}, D_{\mu}^{-}\right\}$, in particular the distorsion of the system $\left\{B_{\mu}^{+}, B_{\mu}^{-}\right\}$in $\Omega_{1}^{-s}(S M)$ is uniformly bounded above for all $\mu \geq \mu_{0}>1 / 4$.

2.4. The construction of the finitely-additive measures. The core of our argument is the following construction of finitely-additive measures on rectifiable arcs.

Theorem 2.1. For any rectifiable arc $\gamma \subset S M$ the following holds. For any Casimir parameter $\mu \in \mathbb{R}^{+} \backslash\{1 / 4\}$ the following limits exist and do not depend on $s>9 / 2$ :

$$
\hat{\beta}_{\mu}^{ \pm}(\gamma):=\lim _{t \rightarrow+\infty} \frac{\hat{\alpha}_{\mu,-s}^{ \pm}\left(g_{t}^{*} \gamma\right)}{\exp \left(\frac{1 \mp \nu}{2} t\right)} ;
$$

For $\mu=1 / 4$ the limits below exist and do not depend on $s>9 / 2$ :

$$
\begin{aligned}
& \hat{\beta}_{1 / 4}^{+}(\gamma):=\lim _{t \rightarrow+\infty} \frac{\left(\hat{\alpha}_{1 / 4,-s}^{+}+\frac{t}{2} \hat{\alpha}_{1 / 4,-s}^{-}\right)\left(g_{t}^{*} \gamma\right)}{\exp \left(\frac{t}{2}\right)}, \\
& \hat{\beta}_{1 / 4}^{-}(\gamma):=\lim _{t \rightarrow+\infty} \frac{\hat{\alpha}_{1 / 4,-s}^{-}\left(g_{t}^{*} \gamma\right)}{\exp \left(\frac{t}{2}\right)} .
\end{aligned}
$$

The convergence in the limits (32), (33) is exponential in the following precise sense. For all $t>0$, let us introduce the rescaled weak unstable length

$$
|\gamma|_{X V, t}:=\int_{\gamma}|\hat{X}|+e^{-t} \int_{\gamma}|\hat{V}|
$$

There exists a constant $C_{s}>0$ such that, for any $\mu \neq 1 / 4$,

$$
\left|\hat{\beta}_{\mu}^{ \pm}(\gamma)-\frac{\hat{\alpha}_{\mu,-s}^{ \pm}\left(g_{t}^{*} \gamma\right)}{\exp \left(\frac{1 \mp \nu}{2} t\right)}\right| \leq \frac{C_{s}}{\left\|B_{\mu}^{ \pm}\right\|_{-s}} \frac{1+|\gamma|_{X V, t}}{\exp \left(\frac{1 \mp \nu}{2} t\right)},
$$


while for $\mu=1 / 4$,

$$
\begin{aligned}
& \left|\hat{\beta}_{1 / 4}^{+}(\gamma)-\frac{\left(\hat{\alpha}_{1 / 4,-s}^{+}+\frac{t}{2} \hat{\alpha}_{1 / 4,-s}^{-}\right)\left(g_{t}^{*} \gamma\right)}{\exp \left(\frac{t}{2}\right)}\right| \leq \frac{C_{s}(1+t)}{\left\|B_{\mu}^{ \pm}\right\|_{-s}} \frac{\left(1+|\gamma|_{X V, t}\right)}{\exp \left(\frac{t}{2}\right)}, \\
& \left|\hat{\beta}_{1 / 4}^{-}(\gamma)-\frac{\hat{\alpha}_{1 / 4,-s}^{-}\left(g_{t}^{*} \gamma\right)}{\exp \left(\frac{t}{2}\right)}\right| \leq \frac{C_{s}(1+t)}{\left\|B_{\mu}^{ \pm}\right\|_{-s}} \frac{\left(1+|\gamma|_{X V, t}\right)}{\exp \left(\frac{t}{2}\right)} .
\end{aligned}
$$

For all Casimir parameters $\mu \in \mathbb{R}^{+}$, the following bound holds:

$$
\left|\hat{\beta}_{\mu}^{ \pm}(\gamma)\right| \leq \frac{C_{s}}{\left\|B_{\mu}^{ \pm}\right\|_{-s}}\left(1+\int_{\gamma}|\hat{X}|+\int_{\gamma}|\hat{U}|+\int_{\gamma}|\hat{V}|\right) .
$$

Proof of Theorem 2.1 The argument is a refinement of the method of [12], §5.3. The main technical improvement consists in replacing the difference equations of [12] by ordinary differential equations. Here we also work in the more general setting of currents instead of distributions.

For any $s>3 / 2$, consider the decomposition of the current $\gamma \in \Omega_{1}^{-s}(S M)$ given by integration along a rectifiable arc. By definition we have:

$$
\gamma=\mathcal{B}^{-s}(\gamma)+\mathcal{R}^{-s}(\gamma)
$$

We are interested in the evolution of this decomposition under the action of the geodesic flow. By the group property of the geodesic flow $\left\{g_{t}\right\}$ and by formula (38), for any $t, \tau \in \mathbb{R}$ we obtain:

$$
g_{t+\tau}^{*} \gamma=g_{\tau}^{*} \mathcal{B}^{-s}\left(g_{t}^{*} \gamma\right)+g_{\tau}^{*} \mathcal{R}^{-s}\left(g_{t}^{*} \gamma\right)=\mathcal{B}^{-s}\left(g_{t+\tau}^{*} \gamma\right)+\mathcal{R}^{-s}\left(g_{t+\tau}^{*} \gamma\right) .
$$

By projection of (39) under $\mathcal{B}_{\mu}^{-s}: \Omega_{1}^{-s}(S M) \rightarrow \mathcal{B}_{U}^{-s}\left(H_{\mu}\right)$ we therefore have

$$
\mathcal{B}_{\mu}^{-s}\left(g_{t+\tau}^{*} \gamma\right)=g_{\tau}^{*} \mathcal{B}_{\mu}^{-s}\left(g_{t}^{*} \gamma\right)+\mathcal{B}_{\mu}^{-s} g_{\tau}^{*} \mathcal{R}_{\mu}^{-s}\left(g_{t}^{*} \gamma\right)
$$

We would like to differentiate the above identity (40) with respect to the parameter $\tau \in \mathbb{R}$. That is made possible by the following technical result whose proof we postpone until $\S \S 5.2$.

Lemma 2.4. For any $s \geq r>7 / 2$, for any rectifiable arc $\gamma$ in $S M$ and for any irreducible component $H_{\mu} \subset L^{2}(S M)$ of Casimir parameter $\mu \in \mathbb{R} \backslash\{0\}$, the current $\mathcal{R}_{\mu}^{-s}(\gamma) \in \Omega_{1}^{-s}\left(H_{\mu}\right)$ has a unique continuous extension $\mathcal{R}_{\mu}^{-s,-r}(\gamma) \in$ $\Omega_{1}^{-r}\left(H_{\mu}\right)$ and the following uniform bound holds. There exists a constant $C_{r, s}>0$ such that

$$
\left\|\mathcal{R}_{\mu}^{-s,-r}(\gamma)\right\|_{-r} \leq C_{s, r}\left(1+\int_{\gamma}|\hat{X}|+\int_{\gamma}|\hat{V}|\right) .
$$

Now, assuming Lemma2.4, we conclude the proof of Theorem 2.1.

Let $s>9 / 2$. By Lemma 2.4, the current $\tilde{\mathcal{R}}_{\mu}^{-s}(\gamma):=\mathcal{R}_{\mu}^{-s,-(s-1)}(\gamma)$ is welldefined, and the following limit exists in the Hilbert space $\Omega_{1}^{-s}\left(H_{\mu}\right)$ :

$$
\lim _{\tau \rightarrow 0} \frac{g_{\tau}^{*} \mathcal{R}_{\mu}^{-s}\left(g_{t}^{*} \gamma\right)-\mathcal{R}_{\mu}^{-s}\left(g_{t}^{*} \gamma\right)}{\tau}=\mathcal{L}_{X} \tilde{\mathcal{R}}_{\mu}^{-s}\left(g_{t}^{*} \gamma\right) \in \Omega_{1}^{-s}\left(H_{\mu}\right)
$$


Thus, by differentiating (40) with respect to $\tau$ at $\tau=0$, we obtain, for all $t \in \mathbb{R}$,

$$
\frac{d}{d t} \mathcal{B}_{\mu}^{-s}\left(g_{t}^{*} \gamma\right)=\mathcal{L}_{X} \mathcal{B}_{\mu}^{-s}\left(g_{t}^{*} \gamma\right)+\mathcal{B}_{\mu}^{-s} \mathcal{L}_{X} \tilde{\mathcal{R}}_{\mu}^{-s}\left(g_{t}^{*} \gamma\right)
$$

We now write the above differential equation in coordinates. We write

$$
\begin{aligned}
\mathcal{B}_{\mu}^{-s}\left(g_{t}^{*} \gamma\right) & =\hat{\alpha}_{\mu,-s}^{+}(t) B_{\mu}^{+}+\hat{\alpha}_{\mu,-s}^{-}(t) B_{\mu}^{-}, \\
\mathcal{B}_{\mu}^{-s} \mathcal{L}_{X} \tilde{\mathcal{R}}_{\mu}^{-s}\left(g_{t}^{*} \gamma\right) & =\rho_{\mu,-s}^{+}(t) B_{\mu}^{+}+\rho_{\mu,-s}^{-}(t) B_{\mu}^{-} .
\end{aligned}
$$

If $\mu \neq 1 / 4$, by Lemma 2.2 and equation (41) we obtain the following formulas:

$$
\frac{d}{d t} \hat{\alpha}_{\mu,-s}^{ \pm}=\frac{1 \mp \nu}{2} \hat{\alpha}_{\mu,-s}^{ \pm}+\rho_{\mu,-s}^{ \pm}
$$

If $\mu=1 / 4$, we obtain the following formulas:

$$
\frac{d}{d t}\left(\begin{array}{c}
\hat{\alpha}_{\mu,-s}^{+} \\
\hat{\alpha}_{\mu,-s}^{-}
\end{array}\right)=\frac{1}{2}\left(\begin{array}{cc}
1 & -1 \\
0 & 1
\end{array}\right)\left(\begin{array}{c}
\hat{\alpha}_{\mu,-s}^{+} \\
\hat{\alpha}_{\mu,-s}^{-}
\end{array}\right)+\left(\begin{array}{c}
\rho_{\mu,-s}^{+} \\
\rho_{\mu,-s}^{-}
\end{array}\right) .
$$

By writing down solutions of the above O.D.E.'s we conclude that, for $\mu \neq 1 / 4$,

$$
\frac{\hat{\alpha}_{\mu,-s}^{ \pm}(t)}{\exp \left(\frac{1 \mp \nu}{2} t\right)}=\hat{\alpha}_{\mu,-s}^{ \pm}(0)+\int_{0}^{t} \rho_{\mu,-s}^{ \pm}(\tau) e^{-\frac{1 \mp \nu}{2} \tau} d \tau
$$

while for $\mu=1 / 4$, after some elementary calculations,

$$
\begin{aligned}
\frac{\hat{\alpha}_{1 / 4,-s}^{+}(t)+\frac{t}{2} \hat{\alpha}_{1 / 4,-s}^{-}(t)}{\exp \left(\frac{t}{2}\right)} & =\hat{\alpha}_{1 / 4,-s}^{+}(0) \\
& +\int_{0}^{t}\left[\rho_{1 / 4,-s}^{+}(\tau)+\frac{\tau}{2} \rho_{1 / 4,-s}^{-}(\tau)\right] e^{-\tau / 2} d \tau ; \\
\frac{\hat{\alpha}_{1 / 4,-s}^{-}(t)}{\exp \left(\frac{t}{2}\right)}=\hat{\alpha}_{1 / 4,-s}^{-}(0) & +\int_{0}^{t} \rho_{1 / 4,-s}^{-}(\tau) e^{-\tau / 2} d \tau .
\end{aligned}
$$

We conclude the argument by proving that the integrals in formulas (45) and (46) are absolutely convergent (as $t \rightarrow+\infty$ ) and are absolutely and uniformly bounded in terms of the transverse lengths of the rectifiable arc $\gamma$ in $S M$.

Since $M$ is a compact hyperbolic surface, the Casimir spectrum of the standard unitary representation of $S L(2, \mathbb{R})$ on $L^{2}(S M)$ is discrete. Thus, by the distorsion Lemma 2.3 and by formula (42), for all $s>9 / 2$ there exists a constant $C_{s}>0$ such that, for all $\mu \in \mathbb{R}^{+}$, the following estimate holds:

$$
\left|\rho_{\mu,-s}^{ \pm}(t)\right| \leq \frac{C_{s}}{\left\|B_{\mu}^{ \pm}\right\|_{-s}}\left\|\mathcal{L}_{X} \tilde{\mathcal{R}}_{\mu}^{-s}\left(g_{t}^{*} \gamma\right)\right\|_{-s} \leq \frac{C_{s}}{\left\|B_{\mu}^{ \pm}\right\|_{-s}}\left\|\tilde{\mathcal{R}}_{\mu}^{-s}\left(g_{t}^{*} \gamma\right)\right\|_{-s+1}
$$

hence by Lemma2.4 there exists a constant $C_{s}^{\prime}>0$ such that

$$
\left|\rho_{\mu,-s}^{ \pm}(t)\right| \leq \frac{C_{s}^{\prime}}{\left\|B_{\mu}^{ \pm}\right\|_{-s}}\left(1+\int_{\gamma}|\hat{X}|+e^{-t} \int_{\gamma}|\hat{V}|\right) .
$$

The above bound (48) immediately implies that the integrals in formulas (45) and (46) are absolutely and uniformly bounded and convergent (as $t \rightarrow+\infty$ ), hence the limits in the left hand side of both formulas exist. The bound (48) also implies 
that such limits are independent of $s>9 / 2$. In fact, by the distorsion Lemma 2.3 and by Lemma 2.4 for any $s \geq r>9 / 2$ there are constants $C_{s}, C_{s, r}>0$ such that, for any rectifiable arc $\gamma$ and for all Casimir parameters $\mu>0$,

$$
\begin{aligned}
& \left\|B_{\mu}^{ \pm}\right\|_{-s}\left|\hat{\alpha}_{\mu,-s}^{ \pm}(t)-\hat{\alpha}_{\mu,-r}^{ \pm}(t)\right| \leq C_{s}\left\|\mathcal{B}_{\mu}^{-s}\left(g_{t}^{*} \gamma\right)-\mathcal{B}_{\mu}^{-r}\left(g_{t}^{*} \gamma\right)\right\|_{-s} \\
& =C_{s}\left\|\mathcal{R}_{\mu}^{-s}\left(g_{t}^{*} \gamma\right)-\mathcal{R}_{\mu}^{-r}\left(g_{t}^{*} \gamma\right)\right\|_{-s} \leq C_{s, r}\left(1+\int_{\gamma}|\hat{X}|+e^{-t} \int_{\gamma}|\hat{V}|\right) .
\end{aligned}
$$

Thus the limits $\hat{\beta}_{\mu}^{ \pm}(\gamma)$ of formulas (32) and (33) exist and are well-defined and the speed of convergence is correctly given by the estimates in formulas (35) and (36).

Finally, the bound in formula (37) follows from the estimate (48) and the following bound. By the distortion Lemma 2.3 and by the Sobolev embdedding theorem, there exist constants $C_{s}^{\prime \prime}, C_{s}^{\prime \prime \prime}>0$ such that, for all Casimir parameters $\mu>0$,

$$
\left|\hat{\alpha}_{\mu,-s}^{ \pm}(0)\right| \leq \frac{C_{s}^{\prime \prime}}{\left\|B_{\mu}^{ \pm}\right\|_{-s}}\|\gamma\|_{-s} \leq \frac{C_{s}^{\prime \prime \prime}}{\left\|B_{\mu}^{ \pm}\right\|_{-s}}\left(1+\int_{\gamma}|\hat{X}|+\int_{\gamma}|\hat{U}|+\int_{\gamma}|\hat{V}|\right) .
$$

The proof of Theorem 2.1 is therefore complete.

2.5. Proof of the main properties (Theorem 1.1). The proof of the theorem requires a stronger estimate on the current $\hat{\beta}_{\mu}$ than the one given above in Theorem 2.1. The following result is a crucial step in that direction as well as in the proof of the invariance under the unstable horocycle.

For any rectifiable arc $\gamma$, let $\Gamma_{w s}(\gamma)$ be the set of all rectifiable arcs obtained projecting the arc $\gamma$ under the unstable horocycle holonomy on any leaf of the weak stable foliation of the geodesic flow. The weak stable foliation of the geodesic flow is the 2-dimensional foliation tangent to the integrable distribution $\{X, U\}$ in the tangent bundle of $S M$.

Lemma 2.5. For any $\mu \in \mathbb{R}^{+}$, for any rectifiable arc $\gamma$ and any $\gamma_{w s} \in \Gamma_{w s}(\gamma)$,

$$
\hat{\beta}_{\mu}^{ \pm}(\gamma)=\hat{\beta}_{\mu}^{ \pm}\left(\gamma_{w s}\right) .
$$

Proof. For any $\gamma_{w s} \in \Gamma_{w s}(\gamma)$ and let $D\left(\gamma_{w s}, \gamma\right)$ be the surface spanned by the trajectories of the unstable horocycle flow projecting $\gamma$ onto $\gamma_{w s}$. The surface $D\left(\gamma_{w s}, \gamma\right)$ is the union of all unstable horocycle arcs $I$ such that the boundary of $I$ is contained in $\gamma_{w s} \cup \gamma$ and the interior of $I$ is disjoint from $\gamma_{w s} \cup \gamma$. The surface $D\left(\gamma_{w s}, \gamma\right)$ defines by integration a current of dimension 2 (and degree 1 ). Let $g_{-t}\left(\gamma_{w s}\right)$ and $g_{-t}(\gamma)$ be the rectifiable arcs which are direct images of $\gamma_{w s}$ and $\gamma$ under the diffeomorphism $g_{-t}: S M \rightarrow S M$ respectively. By definition the arcs $g_{-t}\left(\gamma_{w s}\right)$ and $g_{-t}(\gamma)$ are respectively the support of the currents $g_{t}^{*} \gamma_{w s}$ and $g_{t}^{*} \gamma$. By definition we have the following identity of currents

$$
g_{t}^{*} D\left(\gamma_{w s}, \gamma\right)=D\left(g_{-t}\left(\gamma_{w s}\right), g_{-t}(\gamma)\right) \text {. }
$$

Since the current $\partial D\left(\gamma_{w s}, \gamma\right)-\left(\gamma-\gamma_{w s}\right)$ is composed of two arcs of orbits of the unstable horocycle flow, it follows that

$$
\partial\left[g_{t}^{*} D\left(\gamma_{w s}, \gamma\right)\right]-\left(g_{t}^{*} \gamma-g_{t}^{*} \gamma_{w s}\right)=g_{t}^{*}\left[\partial D\left(\gamma_{w s}, \gamma\right)-\left(\gamma-\gamma_{w s}\right)\right] \rightarrow 0
$$


Lemma 2.6. The area of $g_{-t} D\left(\gamma_{w s}, \gamma\right)$ is uniformly bounded for all $t>0$.

Proof. For $p \in \gamma$, let $\tau(p)>0$ be the length of the unstable horocycle arc lying in $D:=D\left(\gamma_{w s}, \gamma\right)$. By construction the function $\tau: \gamma \rightarrow \mathbb{R}^{+}$is continuous, hence $\tau_{\gamma}:=\sup \{\tau(p) \mid p \in \gamma\}<+\infty$. We write

$$
D=\bigcup_{p \in \gamma} \bigcup_{\tau \in[0, \tau(p)]} h_{\tau}^{V}(p),
$$

whence, letting $d l$ be the length parameter on $S M$, for the area of $D$ we may write

$$
\operatorname{Area}(D)=\int_{\gamma} \tau d l
$$

Since, by formula (51), for any $t \in \mathbb{R}$,

$$
g_{-t} D=\bigcup_{p \in g_{-t} \gamma} \bigcup_{\tau \in\left[0, e^{-t} \tau(p)\right]} h_{\tau}^{V}(p),
$$

and, since Length $\left(g_{-t} \gamma\right) \leq e^{t} \operatorname{Length}(\gamma)$, by formula (52) we have

$$
\operatorname{Area}\left(g_{-t} D\right)=\int_{g_{-t} \gamma} e^{-t} \tau d l \leq \tau_{\gamma} e^{-t} \operatorname{Length}\left(g_{-t} \gamma\right) \leq \tau_{\gamma} \operatorname{Length}(\gamma)
$$

thus the lemma is proved.

It follows from Lemma 2.6 and formula (50) that for any $s>7 / 2$,

$$
\sup _{t>0}\left\|g_{t}^{*} \gamma-g_{t}^{*} \gamma_{w s}\right\|_{-s}<+\infty
$$

hence by continuity of orthogonal projections

$$
\sup _{t>0}\left|\hat{\alpha}_{\mu,-s}^{ \pm}(\gamma)-\hat{\alpha}_{\mu,-s}^{ \pm}\left(\gamma_{w s}\right)\right|<+\infty .
$$

The statement of the Lemma now follows immediately from the definition of the currents $\hat{\beta}_{\mu}^{ \pm}$in the statement of Theorem 2.1

We return to the proof of Theorem 1.1

Additivity. It follows from the definition of $\hat{\beta}_{\mu}^{ \pm}$in the statement of Theorem 2.1 and from the linearity of projections.

Geodesic Scaling. It follows immediately from the definitions in Theorem 2.1 and from the group property of the geodesic flow. In fact, for any $\mu \neq 1 / 4$,

$$
\begin{aligned}
\hat{\beta}_{\mu}^{ \pm}\left(g_{-t} \gamma\right) & =\lim _{\tau \rightarrow+\infty} \frac{\hat{\alpha}_{\mu,-s}^{ \pm}\left(g_{t+\tau}^{*} \gamma\right)}{\exp \left(\frac{1 \mp \nu}{2} \tau\right)} \\
& =e^{\frac{1 \mp \nu}{2} t} \lim _{\tau \rightarrow+\infty} \frac{\hat{\alpha}_{\mu,-s}^{ \pm}\left(g_{t+\tau}^{*} \gamma\right)}{\exp \left(\frac{1 \mp \nu}{2}(t+\tau)\right)}=e^{\frac{1 \mp \nu}{2} t} \hat{\beta}_{\mu}^{ \pm}(\gamma)
\end{aligned}
$$


For $\mu=1 / 4$, the geodesic scaling properties of the current $\hat{\beta}_{1 / 4}^{-}$can be proved as above (as for $\mu \neq 1 / 4$ ), while for the current $\hat{\beta}_{1 / 4}^{+}$, the following holds:

$$
\begin{aligned}
\hat{\beta}_{1 / 4}^{+}\left(g_{-t} \gamma\right) & =\lim _{\tau \rightarrow+\infty} \frac{\left(\hat{\alpha}_{1 / 4,-s}^{+}+\frac{\tau}{2} \hat{\alpha}_{1 / 4,-s}^{-}\right)\left(g_{t+\tau}^{*} \gamma\right)}{\exp \left(\frac{\tau}{2}\right)} \\
& =e^{\frac{t}{2}} \lim _{\tau \rightarrow+\infty} \frac{\left(\hat{\alpha}_{1 / 4,-s}^{+}+\frac{\tau}{2} \hat{\alpha}_{1 / 4,-s}^{-}\right)\left(g_{\tau}^{*} \gamma\right)}{\exp \left(\frac{\tau-t}{2}\right)} \\
& =e^{\frac{t}{2}}\left(\hat{\beta}_{1 / 4}^{+}(\gamma)-\frac{t}{2} \hat{\beta}_{1 / 4}^{-}(\gamma)\right) .
\end{aligned}
$$

Unstable Horocycle Invariance. It follows from Lemma 2.5. In fact, for any rectifiable arc $\gamma$ and for any $t>0$, the arcs $\gamma$ and $h_{t}^{V}(\gamma)$ have common weak stable projections. In other terms, the identity $\Gamma_{w s}(\gamma)=\Gamma_{w s}\left(h_{t}^{V} \gamma\right)$ holds by definition. Let then $\gamma_{w s} \in \Gamma_{w s}(\gamma)=\Gamma_{w s}\left(h_{t}^{V} \gamma\right)$. By Lemma2.5 we have:

$$
\hat{\beta}_{\mu}^{ \pm}\left(h_{t}^{V} \gamma\right)=\hat{\beta}_{\mu}^{ \pm}\left(\gamma_{w s}\right)=\beta_{\mu}^{ \pm}(\gamma)
$$

Hölder property. Let $\gamma_{w s}$ be any rectifiable arc contained in a weak stable manifold of the geodesic flow and let

$$
t=\log \left(\int_{\gamma_{w s}}|\hat{U}|\right) .
$$

By construction the transverse lengths of the rectifiable arc $\gamma_{w s}(t):=g_{t}\left(\gamma_{w s}\right)$ satisfy the following properties:

$$
\int_{\gamma_{w s}(t)}|\hat{X}|=\int_{\gamma_{w s}}|\hat{X}| \quad \text { and } \quad \int_{\gamma_{w s}(t)}|\hat{U}|=1 .
$$

Thus by Theorem 2.1 and by the geodesic scaling properties of the finitely-additive measures $\hat{\beta}^{ \pm}$, the following bounds hold: for all $s>9 / 2$ there exists a constant $C_{s}>0$ such that, for $\mu \neq 1 / 4$, the following bound holds:

$$
\begin{aligned}
\left|\hat{\beta}_{\mu}^{ \pm}\left(\gamma_{w s}\right)\right| & =e^{\frac{1 \mp \operatorname{Re} \nu}{2} t}\left|\hat{\beta}_{\mu}^{ \pm}\left(\gamma_{w s}(t)\right)\right| \\
& \leq \frac{C_{s}}{\left\|B_{\mu}^{ \pm}\right\|_{-s}}\left(1+\int_{\gamma_{w s}}|\hat{X}|\right)\left(\int_{\gamma_{w s}}|\hat{U}|\right)^{\frac{1 \mp \operatorname{Re} \nu}{2}}
\end{aligned}
$$

for $\mu=1 / 4$, the following bounds hold:

$$
\begin{aligned}
& \left|\hat{\beta}_{1 / 4}^{+}\left(\gamma_{w s}\right)\right|=e^{\frac{t}{2}}\left|\left(\hat{\beta}_{1 / 4}^{+}-\frac{t}{2} \hat{\beta}_{1 / 4}^{-}\right)\left(\gamma_{w s}(t)\right)\right| \\
& \leq \frac{C_{s}}{\left\|B_{1 / 4}^{+}\right\|_{-s}}\left(1+\int_{\gamma_{w s}}|\hat{X}|\right)\left(\int_{\gamma_{w s}}|\hat{U}|\right)^{\frac{1}{2}+} ; \\
& \left|\hat{\beta}_{1 / 4}^{-}\left(\gamma_{w s}\right)\right|=e^{\frac{t}{2}}\left|\hat{\beta}_{1 / 4}^{-}\left(\gamma_{w s}(t)\right)\right| \\
& \leq \frac{C_{s}}{\left\|B_{1 / 4}^{-}\right\|_{-s}}\left(1+\int_{\gamma_{w s}}|\hat{X}|\right)\left(\int_{\gamma_{w s}}|\hat{U}|\right)^{\frac{1}{2}} .
\end{aligned}
$$


We recall that we adopt the notation

$$
L^{\frac{1}{2}+}=L^{\frac{1}{2}}(1+|\log L|), \quad \text { for all } L>0 .
$$

Let now $\gamma$ be any rectifiable arc. By the $S L(2, \mathbb{R})$ commutation relations, there exists a rectifiable arc $\gamma_{w s} \in \Gamma_{w s}(\gamma)$ such that

$$
\int_{\gamma_{w s}}|\hat{X}| \leq \int_{\gamma}|\hat{X}|+\int_{\gamma}|\hat{U}| \int_{\gamma}|\hat{V}| \quad \text { and } \quad \int_{\gamma_{w s}}|\hat{U}|=\int_{\gamma}|\hat{U}| .
$$

It follows from estimates (56) and (58) that, for $\mu \neq 1 / 4$,

$$
\left|\hat{\beta}_{\mu}^{ \pm}\left(\gamma_{w s}\right)\right| \leq \frac{C_{s}}{\left\|B_{\mu}^{ \pm}\right\|_{-s}}\left(1+\int_{\gamma}|\hat{X}|+\int_{\gamma}|\hat{U}| \int_{\gamma}|\hat{V}|\right)\left(\int_{\gamma}|\hat{U}|\right)^{\frac{1 \mp \operatorname{Re} \nu}{2}},
$$

while for $\mu=1 / 4(\nu=0)$, by estimates (57) and (58),

$$
\begin{aligned}
& \left|\hat{\beta}_{1 / 4}^{+}\left(\gamma_{w s}\right)\right| \leq \frac{C_{s}}{\left\|B_{1 / 4}^{+}\right\|_{-s}}\left(1+\int_{\gamma}|\hat{X}|+\int_{\gamma}|\hat{U}| \int_{\gamma}|\hat{V}|\right)\left(\int_{\gamma}|\hat{U}|\right)^{\frac{1}{2}+}, \\
& \left|\hat{\beta}_{1 / 4}^{-}\left(\gamma_{w s}\right)\right| \leq \frac{C_{s}}{\left\|B_{1 / 4}^{-}\right\|_{-s}}\left(1+\int_{\gamma}|\hat{X}|+\int_{\gamma}|\hat{U}| \int_{\gamma}|\hat{V}|\right)\left(\int_{\gamma}|\hat{U}|\right)^{\frac{1}{2}} .
\end{aligned}
$$

By Lemma 2.5 the above bounds immdediately implies the Hölder property stated in Theorem 1.1 which is therefore completely proved.

2.6. Proof of weak unstable vanishing (Corollary 1.1). On one hand, by the geodesic scaling property of Theorem 1.1, for any rectifiable arc $\gamma$ in $S M$

$$
\hat{\beta}_{\mu}^{ \pm}(\gamma)=\exp \left(-\frac{1 \mp \nu}{2} t\right) \beta_{\mu}^{ \pm}\left(g_{-t} \gamma\right)
$$

on the other hand, for any rectifiable arc $\gamma_{w u}$ contained in a weak unstable manifold for the geodesic flow, by the Hölder property of Theorem 1.1,

$$
\left|\hat{\beta}_{\mu}^{ \pm}\left(g_{-t} \gamma_{w u}\right)\right| \leq C_{\mu}\left(1+\int_{\gamma}|\hat{X}|\right) .
$$

It follows immediately that $\hat{\beta}_{\mu}^{ \pm}\left(\gamma_{w u}\right)=0$ as stated.

2.7. Proof of existence of dynamical projections (Corollary 1.2). For any $r>$ 0 , let $\mathcal{B}_{+}^{-r}(S M) \subset \Omega_{1}^{-r}(S M)$ be the closed subspace of basic currents for the stable horocycle foliation, supported on irreducible unitary representations of the principal and complementary series, and let $\mathcal{B}_{+}^{-r}: \Omega_{1}^{-r}(S M) \rightarrow \mathcal{B}_{+}^{-r}(S M)$ be the orthogonal projection.

By definition (see formula (31), the orthogonal projection $\mathcal{B}_{+}^{-r}\left(g_{t}^{*} \gamma\right)$ is given by the formula:

$$
\mathcal{B}_{+}^{-r}\left(g_{t}^{*} \gamma\right)=\sum_{\mu \in \operatorname{Spec}(\square) \cap \mathbb{R}^{+}} \hat{\alpha}_{\mu,-r}^{+}\left(g_{t}^{*} \gamma\right) B_{\mu}^{+}+\hat{\alpha}_{\mu,-r}^{-}\left(g_{t}^{*} \gamma\right) B_{\mu}^{-} .
$$

By Lemma 2.2 we have that, for Casimir parameters $\mu \in \mathbb{R}^{+} \backslash\{1 / 4\}$,

$$
g_{-t}^{*}\left(B_{\mu}^{ \pm}\right)=\exp \left(-\frac{1 \mp \nu}{2} t\right) B_{\mu}^{ \pm},
$$


while for $\mu=1 / 4$,

$$
g_{-t}^{*}\left(\begin{array}{l}
B_{1 / 4}^{+} \\
B_{1 / 4}^{-}
\end{array}\right)=\exp \left(-\frac{t}{2}\right)\left(\begin{array}{cc}
1 & 0 \\
\frac{t}{2} & 1
\end{array}\right)\left(\begin{array}{l}
B_{1 / 4}^{+} \\
B_{1 / 4}^{-}
\end{array}\right) .
$$

It follows then from Theorem 2.1 that the series

$$
g_{-t}^{*} \mathcal{B}_{+}^{-r}\left(g_{t}^{*} \gamma\right)=\sum_{\mu \in \operatorname{Spec}(\square) \cap \mathbb{R}^{+}} \hat{\alpha}_{\mu,-r}^{+}\left(g_{t}^{*} \gamma\right) g_{-t}^{*} B_{\mu}^{+}+\hat{\alpha}_{\mu,-r}^{-}\left(g_{t}^{*} \gamma\right) g_{-t}^{*} B_{\mu}^{-}
$$

converges in the distributional sense as $t \rightarrow+\infty$ to the series

$$
\hat{B}(\gamma)=\sum_{\mu \in \operatorname{Spec}(\square) \cap \mathbb{R}^{+}} \hat{\beta}_{\mu}^{+}(\gamma) B_{\mu}^{+}+\hat{\beta}_{\mu}^{-}(\gamma) B_{\mu}^{-} .
$$

In fact, by Theorem 2.1 there exists a constant $C>0$ such that, for all $s \geq r>$ $9 / 2$, for all Casimir parameter $\mu>0$ and for all $t \in \mathbb{R}^{+}$the following bound holds :

$$
\left|\hat{\alpha}_{\mu,-r}^{ \pm}\left(g_{t}^{*} \gamma\right)\right|\left\|g_{-t}^{*} B_{\mu}^{ \pm}\right\|_{-s} \leq C \frac{\left\|B_{\mu}^{ \pm}\right\|_{-s}}{\left\|B_{\mu}^{ \pm}\right\|_{-r}} .
$$

The dual Sobolev norms in the above estimate can be compared as follows: since the distributions $D_{\mu}^{ \pm} \in \mathcal{D}^{\prime}\left(H_{\mu}\right)$ for all $\mu>0$, for any $\sigma \in \mathbb{R}^{+}$,

$$
(1+\mu)^{\frac{\sigma}{2}}\left\|D_{\mu}^{ \pm}\right\|_{-s}=\left\|(I+\square)^{\frac{\sigma}{2}} D_{\mu}^{ \pm}\right\|_{-s} \leq\left\|D_{\mu}^{ \pm}\right\|_{-s+\sigma} .
$$

By the Weyl asymptotics for the Laplace-Beltrami operator on a compact hyperbolic surface, for any $\sigma>1$,

$$
\sum_{\mu \in \operatorname{Spec}(\square) \cap \mathbb{R}^{+}}\left(\frac{1}{1+\mu}\right)^{\sigma}<+\infty,
$$

hence for every $s>r+1>11 / 2$ the series in formula (59) is absolutely uniformly convergent to the current $\hat{B}$ defined in formula (60) in the Sobolev space of currents $\Omega_{1}^{-s}(S M)$. Finally, by the uniform convergence of the series in formula (60), all the properties of the current $\hat{B}(\gamma)$ stated in the corollary (additive property, weak unstable vanishing, unstable horocycle invariance ad Hölder property) follow from the corresponding properties for the finitely additive measures $\hat{\beta}_{\mu}^{ \pm}(\gamma)$ stated in Theorem 1.1 and Corollary 1.1 .

\section{Additive Cocycles And Limit Distributions.}

In this section we prove our results on additive Hölder cocycles for the horocycle flow (Theorem 1.2). We then derive the approximation theorem (Theorem 1.3) and our results on limit distributions of ergodic integrals of the horocycle flow. 
3.1. Proof of the cocycle theorem (Theorem 1.2). Let us recall that the functions $\beta_{\mu}^{ \pm}: S M \times \mathbb{R} \rightarrow \mathbb{C}$ are defined in terms of the finitely additive measures $\hat{\beta}_{\mu}^{ \pm}$on rectifiable arcs. For any $(x, T) \in S M \times \mathbb{R}$, let $\gamma_{U}(x, T)$ be the oriented horocycle $\operatorname{arc}$

$$
\gamma_{U}(x, T):=\left\{h_{t}^{U}(x) \mid t \in[0, T]\right\} .
$$

For every Casimir parameter $\mu>0$, let

$$
\hat{\beta}_{\mu}^{ \pm}(x, T):=\hat{\beta}_{\mu}^{ \pm}\left[\gamma_{U}(x, T)\right] .
$$

By Corollary 1.3 , we derive the following approximation results for functions supported on a single irreducible component. For functions supported on the complementary series we have

Corollary 3.1. For any $\mu \in(0,1 / 4)$, there exists $\varepsilon_{\mu}>0$ such that the following holds. Let $f \in W^{s}\left(H_{\mu}\right)(s>11 / 2)$ be any function such that $D_{\mu}^{-}(f) \neq 0$. Then

$$
\max _{T \in[0,1]}\left|\frac{1}{D_{\mu}^{-}(f) \exp \left(\frac{1+\nu}{2} t\right)} \int_{0}^{T e^{t}} f \circ h_{\tau}^{U}(x) d \tau-\beta_{\mu}^{-}\left(g_{t} x, T\right)\right|=O\left(\exp \left(-\varepsilon_{\mu} t\right)\right) .
$$

Let $f \in W^{s}\left(H_{\mu}\right)(s>11 / 2)$ be any function such that $D_{\mu}^{-}(f)=0$, but $D_{\mu}^{+}(f) \neq 0$. Then

$$
\max _{T \in[0,1]}\left|\frac{1}{D_{\mu}^{+}(f) \exp \left(\frac{1-\nu}{2} t\right)} \int_{0}^{T e^{t}} f \circ h_{\tau}^{U}(x) d \tau-\beta_{\mu}^{+}\left(g_{t} x, T\right)\right|=O\left(\exp \left(-\varepsilon_{\mu} t\right)\right) .
$$

For functions supported on the principal series we have

Corollary 3.2. For all $\mu>1 / 4$, there exists $\varepsilon_{\mu}>0$ such that the following holds. For any function $f \in W^{s}\left(H_{\mu}\right)(s>11 / 2)$,

$$
\begin{gathered}
\max _{T \in[0,1]} \mid\left(\frac{1}{\exp \left(\frac{t}{2}\right)} \int_{0}^{T e^{t}} f \circ h_{\tau}^{U}(x) d \tau-\beta_{\mu}^{+}\left(g_{t} x, T\right) D_{\mu}^{+}(f) \exp \left(-\frac{\nu t}{2}\right)\right. \\
-\beta_{\mu}^{-}\left(g_{t} x, T\right) D_{\mu}^{-}(f) \exp \left(\frac{\nu t}{2}\right) \mid=O\left(\exp \left(-\varepsilon_{\mu} t\right)\right) .
\end{gathered}
$$

For $\mu=1 / 4$, there exists $\varepsilon>0$ such that, for any $f \in W^{s}\left(H_{1 / 4}\right)(s>11 / 2)$,

$$
\begin{aligned}
\max _{T \in[0,1]} \mid\left(\frac{1}{\exp \left(\frac{t}{2}\right)} \int_{0}^{T e^{t}}\right. & f \circ h_{\tau}^{U}(x) d \tau-\beta_{1 / 4}^{+}\left(g_{t} x, T\right) D_{1 / 4}^{+}(f) \\
& -\beta_{1 / 4}^{-}\left(g_{t} x, T\right)\left[D_{1 / 4}^{-}(f)-\frac{t}{2} D_{1 / 4}^{+}(f)\right] \mid=O(\exp (-\varepsilon t)) .
\end{aligned}
$$

We proceed with the proof of Theorem 1.2

Cocycle property. It follows from the additivity property of the measures $\hat{\beta}_{\mu}^{ \pm}$ and from the cocycle properties of horocycle arcs:

$$
\gamma(x, S+T)=\gamma(x, S) \cup \gamma\left(h_{S}^{U} x, T\right), \quad \text { for all }(x, S, T) \in S M \times \mathbb{R}^{2} .
$$

Geodesic scaling. It follows from the geodesic scaling property of the measures $\hat{\beta}_{\mu}^{ \pm}$, since by the commutation relation (3),

$$
g_{-t} \gamma(x, T)=\gamma\left(g_{-t} x, T e^{t}\right), \quad \text { for all }(x, T, t) \in S M \times \mathbb{R}^{2} .
$$


Hölder property. It follows from the Hölder property of the currents $\hat{\beta}_{\mu}^{ \pm}$. In fact, if $\gamma:=\gamma(x, T)$ is a stable horocycle arc, then

$$
\left|\int_{\gamma} \hat{U}\right|=|T|, \quad \int_{\gamma} \hat{X}=\int_{\gamma} \hat{V}=0 .
$$

Orthogonality. Take $T \in \mathbb{R}$. By Corollaries 3.1 and 3.2 , the function $\beta_{\mu}^{ \pm}(\cdot, T)$ is the uniform limit of normalized ergodic integrals, i.e., continuous functions lying in the space $H_{\mu}$, and so the function $\beta_{\mu}^{ \pm}(\cdot, T)$ must itself belong to $H_{\mu} \subset L^{2}(S M)$.

3.2. Proof of the approximation theorem (Theorem 1.3). For all rectifiable arcs $\gamma \subset S M$ and for all Casimir parameters $\mu>0$, let $\hat{B}_{\mu}(\gamma) \in \mathcal{B}_{U}^{-s}(S M)$ be the basic current defined as follows:

$$
\hat{B}_{\mu}(\gamma):=\hat{\beta}_{\mu}^{+}(\gamma) B_{\mu}^{+}+\hat{\beta}_{\mu}^{-}(\gamma) B_{\mu}^{-} .
$$

It follows from the bounds (35) and (36) in Theorem 2.1 that, for any $r>9 / 2$ there exists a constant $C_{r}>0$ such that, for all $t \geq 0$,

$$
\left\|\left(\mathcal{B}_{\mu}^{-r} \circ g_{t}^{*}\right)(\gamma)-\left(\hat{B}_{\mu} \circ g_{t}^{*}\right)(\gamma)\right\|_{-r} \leq C_{r}\left(1+\int_{\gamma}|\hat{X}|+e^{-t} \int_{\gamma}|\hat{V}|\right) .
$$

By Lemma 2.4 and from the splitting formula (38) there exists a constant $C_{r}^{\prime}>0$ such that, for all $t \geq 0$,

$$
\left\|\left(\mathcal{B}_{\mu}^{-r} \circ g_{t}^{*}\right)(\gamma)-g_{t}^{*}(\gamma)\right\|_{-r} \leq C_{r}^{\prime}\left(1+\int_{\gamma}|\hat{X}|+e^{-t} \int_{\gamma}|\hat{V}|\right) .
$$

Let $\gamma_{+}$denote the projection of the current $\gamma$ onto the components of the principal and complementary series. By orthogonality and by the Weyl asymptotics for hyperbolic surfaces, for any $s>r+1$, there exists $C_{s}>0$ such that

$$
\left\|g_{t}^{*}\left(\gamma_{+}\right)-\left(\hat{B} \circ g_{t}^{*}\right)(\gamma)\right\|_{-s} \leq C_{s}\left(1+\int_{\gamma}|\hat{X}|+e^{-t} \int_{\gamma}|\hat{V}|\right) .
$$

Taking $t=0$, we obtain Theorem 1.3. In fact, for any 1-form $\lambda \in \Omega_{1}^{s}(S M)(s>$ $11 / 2$ ) supported on the irreducible components of the principal and complementary series we have

$$
\left|\int_{\gamma} \lambda-\hat{B}_{\lambda}(\gamma)\right|=\left|<\gamma_{+}-\hat{B}(\gamma), \lambda>\right| \leq C_{s}\|\lambda\|_{s}\left(1+\int_{\gamma}|\hat{X}|+\int_{\gamma}|\hat{V}|\right) .
$$

The proof is complete.

3.3. Proof of the limit theorems: complementary series (Theorem 1.4). We now assume that our hyperbolic surface admits complementary series, that is, the spectrum of the Laplace operator has eigenvalue in the open interval $(0,1 / 4)$. Let $s>11 / 2$ and consider smooth functions with non-trivial projection on the complementary series components. Let $\mu_{f} \in(0,1)$ be the smallest Casimir parameter appearing (non-trivially) in the decomposition of a zero-average function $f \in C^{\infty}(S M)$. Let $\nu_{f}:=\sqrt{1-4 \mu_{f}}$. Let $H_{1}, \ldots, H_{k} \subset L^{2}(S M)$ be the collection of all irreducible components of Casimir parameters $\mu_{1}=\cdots=\mu_{k}=\mu_{f}$ and let $\left\{D_{1}^{ \pm}, \ldots, D_{k}^{ \pm}\right\}$be the basis of eigenvectors of the geodesic flow of the space 
of invariant distributions supported on $W^{s}\left(H_{1} \oplus \cdots \oplus H_{k}\right)$ and let $\beta^{ \pm}, \ldots, \beta_{k}^{ \pm}$: $S M \times \mathbb{R} \rightarrow \mathbb{C}$ be the corresponding cocycles for the horocycle flow.

The main step in the proof is the following approximation Lemma which immediately follows from the approximation theorem (Theorem 1.3).

Lemma 3.1. There exists $\alpha>0$ such that the following holds. For every $s>11 / 2$ there exists a constant $C_{s}>0$ such that, for every function $f \in W^{s}(S M)$ of zero average, for all $(x, T) \in S M \times \mathbb{R}$ and $t>0$,

$$
\left|\frac{1}{e^{\frac{1+\nu_{f}}{2} t}} \int_{0}^{T e^{t}} f \circ h_{\tau}^{U}(x) d \tau-\sum_{i=1}^{k} D_{i}^{-}(f) \beta_{i}^{-}\left(g_{t} x, T\right)\right| \leq C_{s}\|f\|_{s} e^{-\alpha t} .
$$

The cocycles $\beta_{1}^{-}, \ldots, \beta_{k}^{-}$(in fact, all cocycles $\beta_{\mu}^{ \pm}$) have zero average but are not identically zero on $S M$. It follows that, for all $i \in\{1, \ldots, k\}$, we have

$$
\left\|\beta_{i}^{-}(\cdot, T)\right\| \neq 0, \quad \text { for all } T \in \mathbb{R} \backslash\{0\} .
$$

By the orthogonality property of Theorem 1.2 , the random variables

$$
\beta_{1}^{-}(\cdot, T), \ldots, \beta_{k}^{-}(\cdot, T)
$$

are orthogonal (uncorrelated). By Lemma 3.1 for any $s>11 / 2$ and for any function $f \in W^{s}(S M)$ of zero average such that $\left(D_{1}^{-}(f), \ldots, D_{k}^{-}(f)\right) \neq(0, \ldots, 0)$, we have

$$
\left|\frac{\left\|\int_{0}^{T e^{t}} f \circ h_{t}^{U}(x) d t\right\|}{e^{\frac{1+\nu_{f}}{2} t}\left(\sum_{i=1}^{k}\left|D_{i}^{-}(f)\right|^{2}\left\|\beta_{i}^{-}(\cdot, T)\right\|^{2}\right)^{1 / 2}}-1\right| \leq C_{s} e^{-\alpha t} .
$$

Finally, by Lemma 3.1 and by definition of the Lévy-Prohorov metric [3], for all $T \in[0,1]$ and all $t>0$, we have

$$
d_{L P}\left(\mathfrak{M}_{t}(f, T), P_{c p}(f, T)\right) \leq C_{s}\|f\|_{s} e^{-\alpha t} .
$$

The Theorem is proved.

3.4. Proof of the limit theorems: principal series (Theorem 1.5). We turn next to limit theorems for functions supported on the principal series. We prove our main theorem (Theorem 1.5) on the asymptotics of probability distributions of normalized ergodic integrals and derive our conditional results on the uniqueness of the limit distributions (Corollary 1.4 and Corollary 1.5).

Let us recall that, by construction, for any Casimir parameter $\mu>1 / 4$,

$$
D_{\mu}^{-}=\overline{D_{\mu}^{+}} \quad \text { and } \quad \beta_{\mu}^{-}=\overline{\beta_{\mu}^{+}} \text {. }
$$

It follows that for any real-valued function $f \in W^{s}\left(H_{\mu}\right)$,

$$
\beta_{f}(x, T)=\operatorname{Re}\left[D_{\mu}^{+}(f) \beta_{\mu}^{+}(x, T)\right], \quad \text { for all }(x, T) \in S M \times \mathbb{R} .
$$

Let $\left\{\mu_{n}\right\}$ be the sequence of Casimir parameter in the interval $(1 / 4,+\infty)$ (listed with multiplicities), let $\left\{D_{\mu_{n}}^{ \pm}\right\}$denote the sequence of normalized horocycle invariant distributions and let $\left\{\beta_{\mu_{n}}^{ \pm}\right\}$denote the corresponding sequence of additive 
Hölder cocycles. For any sequence $\mathbf{z} \in \ell^{1}(\mathbb{N}, \mathbb{C})$, let $\beta_{\mathbf{z}}: S M \times \mathbb{R} \rightarrow \mathbb{R}$ be the Hölder additive cocycle for the horocycle flow defined as follows:

$$
\beta_{\mathbf{z}}:=\operatorname{Re}\left[\sum_{n \in \mathbb{N}} z_{n} \beta_{\mu_{n}}^{+}\right]=\sum_{n \in \mathbb{N}}\left(z_{n} \beta_{\mu_{n}}^{+}+\bar{z}_{n} \beta_{\mu_{n}}^{-}\right) .
$$

It follows from Theorem 1.2 , in particular from the uniform bound on additive cocycles given in the Hölder property, that the series in formula (67) is convergent for any $\mathbf{z} \in \ell^{1}(\mathbb{N}, \mathbb{C})$, hence the additive cocycle $\beta_{\mathbf{z}}$ is well-defined. By the orthogonality property of the system $\left\{\beta_{\mu_{n}}^{+}\right\}$of additive cocycles, it follows that, for any $\mathbf{z} \in \ell^{1}(\mathbb{N}, \mathbb{C}) \backslash\{0\}$, the zero-average function $\beta_{\mathbf{z}}(\cdot, T)$ is non-constant, hence

$$
\left\|\beta_{\mathbf{z}}(\cdot, T)\right\| \neq 0, \quad \text { for all } T>0 .
$$

Let $s>11 / 2$. For any real-valued function $f \in W^{s}(S M)$ supported on irreducible components of the principal series, we have

$$
\beta_{f}(x, T)=\operatorname{Re}\left[\sum_{n \in \mathbb{N}} D_{\mu_{n}}^{+}(f) \beta_{\mu_{n}}^{+}(x, T)\right], \quad \text { for all }(x, T) \in S M \times \mathbb{R} .
$$

Theorem 1.5 follows from the following lemma that can in turn be derived from the approximation theorem (Theorem 1.3 ). For all $n \in \mathbb{N}$, let $v_{n}:=\sqrt{4 \mu_{n}-1} \in \mathbb{R}^{+}$.

Lemma 3.2. For every $s>11 / 2$ there exists $C_{s}>0$ such that, for any real-valued function $f \in W^{s}\left(H_{\mu}\right)$ supported on irreducible components of the principal series, for all $(x, T) \in S M \times \mathbb{R}$ and $t>0$, we have

$$
\left|\frac{1}{e^{\frac{t}{2}}} \int_{0}^{T e^{t}} f \circ h_{\tau}^{U}(x) d \tau-\operatorname{Re}\left[\sum_{n \in \mathbb{N}} D_{\mu_{n}}^{+}(f) e^{\frac{i v_{n} t}{2}} \beta_{\mu_{n}}^{+}\left(g_{t} x, T\right)\right]\right| \leq C_{s}\|f\|_{s} e^{-\frac{t}{2}} .
$$

By Theorem 1.5 for real-valued functions supported on the principal series limit distributions exist along time sequences such that the orbit of the toral translation of frequency $v / 2 \in \mathbb{R}^{\infty}$ on the infinite torus $\mathbb{T}^{\infty}$ converges. Conjecturally the limit does not exist otherwise. However, we are not able to prove that the limit distribution does not exist for any function and any time sequence. Nevertheless, as a straightforward consequence of Theorem 1.5 , we derive the following result.

Corollary 3.3. Let $f \in C^{\infty}(S M)$ be any real-valued function supported on the irreducible components of the principal series. If the family probability distributions $\mathfrak{M}_{t}(f, T)$ has a (unique) limit as $t \rightarrow+\infty$ for some $T \in[0,1]$, then for all $T \in[0,1]$ the family of probability distributions $P_{p r}(f, \cdot, T)$ is constant on any minimal set of the linear flow of frequency $v / 2 \in \mathbb{R}^{\infty}$ on the infinite torus $\mathbb{T}^{\infty}$.

Finally, Corollary 1.4 and Corollary 1.5 follow from the above Corollary 3.3 and, respectively, from Lemma 5.8 and Lemma 5.9 in Section 5.4.

By Theorem 5.2, proved in Section 5.3, for every $n \in \mathbb{N}$ there exists $\theta_{n}^{*} \in \mathbb{T}$ such that the cocycle $\beta_{n}:=e^{i \theta_{n}^{*}} \beta_{\mu_{n}}^{+}$has the property that, for all $T \in \mathbb{R}$,

$$
<\beta_{n}(\cdot, T), \overline{\beta_{n}}(\cdot, T)>=e^{2 i \theta_{n}^{*}}<\beta_{\mu_{n}}^{+}(\cdot, T), \beta_{\mu_{n}}^{-}(\cdot, T)>\in \mathbb{R}^{+} .
$$

Let us assume that there exists a real-valued function $f \in W^{S}(S M)$ supported on finitely many irreducible components $H_{1}, \ldots, H_{m}$ of the principal series such 
that, for some $T>0$, the probability distribution $P_{p r}(f, \cdot, T)$ is constant on a $d$-dimensional subtorus $\mathbb{T}^{d}$ of the infinite dimensional torus $\mathbb{T}^{\infty}$. If the Casimir spectrum is simple on $H_{1} \oplus \cdots \oplus H_{n}$, then there exist distinct integral vectors $v^{(1)}, \ldots, v^{(m)} \in \mathbb{R}^{d}$ such that the probability distributions of the random variables

$$
\frac{\operatorname{Re}\left[\sum_{k=1}^{m}\left|D_{k}^{+}(f)\right| e^{i<v^{(k)}, \theta>} \beta_{k}(\cdot, T)\right]}{\left\|\operatorname{Re}\left[\sum_{k=1}^{m}\left|D_{k}^{+}(f)\right| e^{i<v^{(k)}, \theta>} \beta_{k}(\cdot, T)\right]\right\|} .
$$

does not depend on $\theta \in \mathbb{T}^{d}$. By formula (68), we have

$$
\begin{aligned}
A_{k}^{2}:= & \left\|\operatorname{Re} \beta_{k}(\cdot, T)\right\|^{2}>B_{k}^{2}:=\left\|\operatorname{Im} \beta_{k}(\cdot, T)\right\|^{2} ; \\
& <\operatorname{Re} \beta_{k}(\cdot, T), \operatorname{Im} \beta_{k}(\cdot, T)>=0 .
\end{aligned}
$$

Thus by the orthogonality property, a calculation yields

$$
\begin{aligned}
& \left\|\operatorname{Re}\left[\sum_{k=1}^{m}\left|D_{k}^{+}(f)\right| e^{i<v^{(k)}, \theta>} \beta_{k}(\cdot, T)\right]\right\|_{0}^{2}= \\
& \sum_{k=1}^{m}\left|D_{k}^{+}(f)\right|^{2}\left(A_{k}^{2} \cos ^{2}<v^{(k)}, \theta>+B_{k}^{2} \sin ^{2}<v^{(k)}, \theta>\right),
\end{aligned}
$$

hence Lemma 5.8 and Lemma 5.9 in Section 5.4 do apply to the family of probability distributions in formula (69). Corollary 1.4 and Corollary 1.5 follow.

\section{Duality and Classification.}

In this section we prove the duality theorem (Theorem 1.7) and we derive the classification theorem for finitely-additive measures on rectifiable arcs (Theorem 1.8). We conclude with a short discussion of the relations between finitely additive measures on rectifiable arcs and the induced cocycles for the horocycle flow on one hand, and invariant conformal distributions on the boundary of the Poincaré disk (see [9]).

4.1. Proof of the duality theorems (Theorem 1.6 and Theorem 1.7). Recall that any 1-dimensional, finitely-additive measures can be naturally interpreted as currents of dimension 2 (and degree 1 ).

Lemma 4.1. For any 1-dimensional, finitely-additive measure $\hat{\beta} \in \hat{\mathfrak{B}}_{V}(S M)$ there exists a $V$-invariant distribution $\hat{D}_{\beta} \in \mathcal{J}_{V}(S M)$ such that we have the following identity of currents:

$$
\hat{\beta}=\hat{D}_{\beta} \wedge \hat{U} .
$$

Proof. Let $\hat{\beta} \in \mathcal{B}_{V}(S M)$. We recall that the 2-dimensional current $\hat{\beta}$ is defined by Riemann integration as follows: for all smooth 2 -form $\eta \in \Omega_{2}^{\infty}(S M)$,

$$
<\hat{\beta}, \eta>:=\int_{S M} \hat{\beta} \otimes \eta .
$$

By the weak unstable vanishing property (property (2) in Definition 1.1), the current $\hat{\beta}$ has zero contraction on the vector fields $X, V$. In fact, for any smooth 
3 -form $\omega$ on $S M$, the restrictions of the 2 -forms $\imath_{X} \omega$ and $\imath_{V} \omega$ to any leaf of the weak unstable foliation (tangent to the integrable distribution $\{X, V\}$ ) vanish. It follows that, for all smooth 3 -form $\omega$ on $S M$,

$$
\begin{aligned}
& <\imath_{X} \hat{\beta}, \omega>=\int_{S M} \hat{\beta} \otimes \imath_{X} \omega=0 ; \\
& <\imath_{V} \hat{\beta}, \omega>=\int_{S M} \hat{\beta} \otimes \imath_{V} \omega=0 .
\end{aligned}
$$

Since the dual forms $\{\hat{X}, \hat{U}, \hat{V}\}$ yield a frame of the cotangent bundle, it follows from the identities $\imath_{X} \hat{\beta}=\imath_{V} \hat{\beta}=0$, that there exists a distribution $\hat{D}_{\beta} \in \mathcal{D}^{\prime}(S M)$ such that

$$
\hat{\beta}=\hat{D}_{\beta} \wedge \hat{U} .
$$

In fact, any current of dimension 2 and degree 1 can be written as a linear combination of the 1 -forms $\{\hat{X}, \hat{U}, \hat{V}\}$ with distributional coefficients.

We claim that the distribution $\hat{D}_{\beta}$ is $V$-invariant. By the property of unstable horocycle invariance (property (3) in Definition 1.1) the current $\hat{\beta}=\hat{D}_{\beta} \wedge \hat{U}$ is invariant under the unstable horocycle flow $\left\{h_{t}^{V}\right\}$, hence

$$
0=\mathcal{L}_{V}\left(\hat{D}_{\beta} \wedge \hat{U}\right)=\left(\mathcal{L}_{V} \hat{D}_{\beta}\right) \wedge \hat{U}+\hat{D}_{\beta} \wedge\left(\mathcal{L}_{V} \hat{U}\right) .
$$

A straightforward calculation yields $\mathcal{L}_{V} \hat{U}=0$. Indeed, first write

$$
\mathcal{L}_{V} \hat{U}=\imath_{V} d \hat{U}+d \imath_{V} \hat{U}=\imath_{V} d \hat{U} .
$$

By a standard formula, for any pair of vector fields $W_{1}, W_{2}$, we have

$$
d \hat{U}\left(W_{1}, W_{2}\right)=W_{1} \hat{U}\left(W_{2}\right)-W_{2} \hat{U}\left(W_{1}\right)-\hat{U}\left(\left[W_{1}, W_{2}\right]\right) .
$$

Recall that $U$, being the infinitesimal generator of the stable horocycle, satisfies the commutation relation $[X, U]=U$ (see (1)), whence

$$
d \hat{U}(X, U)=1 \quad \text { and } \quad d \hat{U}(X, V)=d \hat{U}(U, V)=0 .
$$

We have derived the identity

$$
d \hat{U}=-\hat{X} \wedge \hat{U}
$$

which implies that $\mathcal{L}_{V} \hat{U}=\imath_{V} d \hat{U}=0$, as stated.

Formula (74) then implies that $\mathcal{L}_{V} \hat{D}_{\beta} \wedge \hat{U}=0$, hence $\mathcal{L}_{V} \hat{D}_{\beta}=0$, that is, the distribution $\hat{D}_{\beta}$ are $V$-invariant.

We now complete the proof of the duality theorem (Theorem 1.7). By Theorem 1.1 and by Corollary 1.1, the measures $\hat{\beta}_{\mu}^{ \pm}$belong to the space $\hat{\mathfrak{B}}_{V}(S M)$. By Lemma 4.1, there exists $V$-invariant distributions $\hat{D}_{\mu}^{ \pm} \in \mathcal{J}_{V}(S M)$ such that

$$
\hat{\beta}_{\mu}^{ \pm}=\hat{D}_{\mu}^{ \pm} \wedge \hat{U}
$$

Finally we prove that the $V$-invariant distributions $\hat{D}_{\mu}^{ \pm}$are eigenvectors for the 
action of the geodesic flow. An immediate computation yields

$$
g_{t}^{*}(\hat{U})=e^{-t} \hat{U}, \quad \text { for all } t \in \mathbb{R} .
$$

By Theorem 1.1, for $\mu \neq 1 / 4$, we have the following identity of currents

$$
g_{t}^{*}\left(\hat{\beta}_{\mu}^{ \pm}\right)=\exp \left(-\frac{1 \mp \nu}{2} t\right) \hat{\beta}_{\mu}^{ \pm}, \quad \text { for all } t \in \mathbb{R}
$$

while for $\mu=1 / 4(\nu=0)$ and all $t \in \mathbb{R}$, we have

$$
g_{t}^{*}\left(\begin{array}{l}
\hat{\beta}_{1 / 4}^{+} \\
\hat{\beta}_{1 / 4}^{-}
\end{array}\right)=\exp \left(-\frac{t}{2}\right)\left(\begin{array}{cc}
1 & \frac{t}{2} \\
0 & 1
\end{array}\right)\left(\begin{array}{l}
\hat{\beta}_{1 / 4}^{+} \\
\hat{\beta}_{1 / 4}^{-}
\end{array}\right) .
$$

It follows that, for $\mu \neq 1 / 4$,

$$
g_{t}^{*} \hat{D}_{\mu}^{ \pm}=\exp \left(\frac{1 \pm \nu}{2} t\right) \hat{D}_{\mu}^{ \pm}, \quad \text { for all } t \in \mathbb{R}
$$

and finally, for $\mu=1 / 4$ and all $t \in \mathbb{R}$,

$$
g_{t}^{*}\left(\begin{array}{l}
\hat{D}_{1 / 4}^{+} \\
\hat{D}_{1 / 4}^{-}
\end{array}\right)=\exp \left(\frac{t}{2}\right)\left(\begin{array}{cc}
1 & \frac{t}{2} \\
0 & 1
\end{array}\right)\left(\begin{array}{c}
\hat{D}_{1 / 4}^{+} \\
\hat{D}_{1 / 4}^{-}
\end{array}\right),
$$

The proof of the duality theorem is complete.

Remark 4.1. The currents $\hat{\beta}_{\mu}^{ \pm}$are not closed! In fact, for all $\mu \neq 1 / 4$ we have

$$
d \hat{\beta}_{\mu}^{ \pm}=\frac{(1 \mp \nu)}{2} \hat{\beta}_{\mu}^{ \pm} \wedge \hat{X}
$$

and, for $\mu=1 / 4(\nu=0)$, we have

$$
d\left(\begin{array}{l}
\hat{\beta}_{1 / 4}^{+} \\
\hat{\beta}_{1 / 4}^{-}
\end{array}\right)=\frac{1}{2}\left(\begin{array}{cc}
1 & -1 \\
0 & 1
\end{array}\right)\left(\begin{array}{l}
\hat{\beta}_{1 / 4}^{+} \\
\hat{\beta}_{1 / 4}^{-}
\end{array}\right) \wedge \hat{X} .
$$

Proof. By (75), since $\hat{D}_{\mu}^{ \pm}$are $V$-invariant distributions, for $\mu \neq 1 / 4$ we have

$$
\begin{aligned}
d\left(\hat{D}_{\mu}^{ \pm} \wedge \hat{U}\right) & =d \hat{D}_{\mu}^{ \pm} \wedge \hat{U}+\hat{D}_{\mu}^{ \pm} \wedge d \hat{U} \\
& =\left(\mathcal{L}_{X} \hat{D}_{\mu}^{ \pm}-\hat{D}_{\mu}^{ \pm}\right) \hat{X} \wedge \hat{U}=\frac{(1 \mp \nu)}{2}\left(\hat{D}_{\mu}^{ \pm} \hat{U}\right) \wedge \hat{X}
\end{aligned}
$$

which is precisely formula (76). Similarly, for $\mu=1 / 4$,

$$
\begin{aligned}
& d\left(\hat{D}_{1 / 4}^{+} \wedge \hat{U}\right)=\left(\mathcal{L}_{X} \hat{D}_{1 / 4}^{+}-\hat{D}_{1 / 4}^{+}\right) \hat{X} \wedge \hat{U}=\left(\frac{1}{2} D_{1 / 4}^{+} \hat{U}-\frac{1}{2} D_{1 / 4}^{-} \hat{U}\right) \wedge \hat{X} \\
& d\left(\hat{D}_{1 / 4}^{-} \wedge \hat{U}\right)=\left(\mathcal{L}_{X} \hat{D}_{1 / 4}^{-}-\hat{D}_{1 / 4}^{-}\right) \hat{X} \wedge \hat{U}=\frac{1}{2}\left(\hat{D}_{1 / 4}^{-} \hat{U}\right) \wedge \hat{X}
\end{aligned}
$$

which yields formula (77). 
4.2. Proof of the classification theorem (Theorem 1.8). By Lemma4.1, for any $\hat{\beta} \in \hat{\mathfrak{B}}_{V}(S M)$ there exists a $V$-invariant distribution $\hat{D}_{\beta} \in \mathcal{J}_{V}(S M)$ such that the following identity holds in the sense of currents:

$$
\hat{\beta}=\hat{D}_{\beta} \wedge \hat{U} \text {. }
$$

By the Hölder property (property (4) in Definition 1.1), there exists $\alpha>0$ and a constant $C>0$ such that for any rectifiable arc $\gamma$ of length not exceeding 1 we have

$$
\left|\hat{\beta}\left(g_{t} \gamma\right)\right| \leq C e^{-\alpha t}, \quad \text { for all } t \in \mathbb{R} .
$$

It follows by the above formulas that for all $s>3 / 2$ there exists $C_{s}>0$ such that

$$
\left\|g_{t}^{*} \hat{D}_{\beta}\right\|_{-s} \leq C_{s} e^{(1-\alpha) t}, \quad \text { for all } t \in \mathbb{R} .
$$

The results of [12] yield a complete classification of all $U$-invariant and, equivalently, of all $V$-invariant distributions by constructing a basis of generalized distributional eigenvectors for the action of geodesic flow on $\mathcal{J}_{U}(S M)$ and $\mathcal{J}_{V}(S M)$. For any $V$-invariant distribution $D_{\mu}$ supported on an irreducible subrepresentation of the discrete series of Casimir parameter $\mu=-n^{2}+n\left(n \in \mathbb{Z}^{+}\right)$, the action of the geodesic flow is given by the formula (see [12])

$$
g_{t}^{*} \hat{D}_{\mu}=e^{n t} \hat{D}_{\mu}, \quad \text { for all } t \in \mathbb{R},
$$

which is not compatible with the bound in formula (80) for $t>0$ large. It follows that the distribution $\hat{D}_{\beta} \in \mathcal{J}_{V}(S M)$ is supported on irreducible unitary subrepresentations of the principal and complementary series.

By the duality theorem, for any $s>3 / 2$, there is a bounded (in fact, isometric) linear map J from the span $\hat{\mathcal{B}}_{+}^{s}(S M)$ of the system

$$
\left\{\hat{\beta}_{\mu}^{ \pm} \mid \mu \in \operatorname{Spec}(\square) \cap \mathbb{R}^{+}\right\} \subset \Omega_{2}^{-s}(S M)
$$

into the Sobolev space $W^{-s}(S M)$. The map J is defined as follows. For a current $\hat{\beta}_{\mu}^{ \pm} \in \hat{\mathcal{B}}_{+}^{s}(S M)$ introduce a current

$$
\mathcal{J}\left(\hat{\beta}_{\mu}^{ \pm}\right)=\hat{X} \wedge \hat{\beta}_{\mu}^{ \pm} \wedge \hat{V}
$$

It is immediate from the definitions of the Sobolev norms that the map $\mathcal{J}$ is isometric on the system $\left\{\hat{\beta}_{\mu}^{ \pm} \mid \mu \in \operatorname{Spec}(\square) \cap \mathbb{R}^{+}\right\}$with respect to the Sobolev norms on the space $\Omega_{2}^{-s}(S M)$ of 2-dimensional current into the space $W^{-s}(S M)$ of distributions, hence it can be extended by linearity and continuity to an isometry defined on the space $\hat{\mathcal{B}}_{+}^{s}(S M)$. We claim that the range of the isometry $\mathcal{J}$ coincides with the space $\mathcal{J}_{V,+}^{s}(S M)$ of all $V$-invariant distributions supported on the principal and complementary series. In fact, the map $\mathcal{J}$ is injective, hence by a dimension argument it is also surjective from the finite dimensional space $\hat{\mathcal{B}}_{\mu}^{s}(S M):=$ $\hat{\mathcal{B}}_{+}^{s}(S M) \cap \Omega_{2}^{-s}\left(H_{\mu}\right)$ onto the space $\mathcal{J}_{V, \mu}^{s}(S M):=\mathcal{J}_{V}(S M) \cap W^{-s}\left(H_{\mu}\right)$, for any fixed Casimir parameter $\mu \in \operatorname{Spec}(\square) \cap \mathbb{R}^{+}$.

We can thus conclude that, since for every functional $\hat{\beta} \in \mathcal{B}_{V}(S M)$, the distribution $\hat{D}_{\beta} \in \mathcal{J}_{V,+}^{s}(S M)$ and since the isometry $\mathcal{J}$ maps $\hat{\mathcal{B}}_{+}^{s}(S M)$ onto $\mathcal{J}_{V,+}^{s}(S M)$, 
the image $\mathcal{B}_{V}^{s}(S M)$ of the space $\mathcal{B}_{V}(S M)$ in $\Omega^{-s}(S M)$ coincide with the span $\hat{\mathcal{B}}_{+}^{s}(S M)$ of the system $\left\{\hat{\beta}_{\mu}^{ \pm} \mid \mu \in \operatorname{Spec}(\square) \cap \mathbb{R}^{+}\right\}$, as stated.

4.3. $\Gamma$-invariant conformal distributions. We now describe the relation between the finitely additive measures $\hat{\beta}_{\mu}^{ \pm}$(lifted to the Lobachevsky plane) and the $\Gamma$ invariant conformal distributions on the boundary of the Poincaré disk.

Let $\mathcal{L}$ denote the Lebesgue measure on the circle $S^{1}$ seen as the boundary of the Poincaré disk $D$. Given a Fuchsian group $\Gamma$, let

$$
\rho_{g}=\frac{d g^{-1} \mathcal{L}}{d \mathcal{L}}, \quad g \in \Gamma
$$

be the Radon-Nikodym multiplicative coycle for the action of $\Gamma$ on $\left(S^{1}, \mathcal{L}\right)$.

Given any complex number $\sigma \in \mathbb{C}$, following S.Cosentino [9] we let ${ }^{\Gamma} \mathcal{D}_{\sigma}^{\prime}\left(S^{1}\right)$ be the space of $\Gamma$-invariant conformal distributions with exponent $\sigma$, that is, those distributions $\phi \in \mathcal{D}^{\prime}\left(S^{1}\right)$ such that

$$
g \phi=\rho_{g}^{-\sigma} \cdot \phi, \quad \text { for any } g \in \Gamma .
$$

As explained in [9], there is a natural (linear) identification between $\Gamma$-invariant conformal distributions of exponent $\sigma \in \mathbb{C}$ and invariant distributions for the stable/unstable horocycle on the quotient $\Gamma \backslash P S L(2, \mathbb{R})$ which are eigenvectors of the geodesic flow of eigenvalue $\sigma-1$.

In fact, let $h^{U}, h^{V}$ denote the stable, resp. unstable horocycle subgroups of $P S L(2, R)$. To any $U$-invariant $[V$-invariant $]$ distribution $D_{U}\left[D_{V}\right]$ on the space $\Gamma \backslash P S L(2, \mathbb{R})$ there correspond $\Gamma$-invariant distribution $\tilde{D}_{U}\left[\tilde{D}_{V}\right]$ on the space of stable [unstable] horocycles $P S L(2, \mathbb{R}) / h^{U}\left[P S L(2, \mathbb{R}) / h^{V}\right]$, (by the the socalled $K A N$ decomposition of $P S L(2, \mathbb{R})$ such space can be identified with the space $K A$ ). If $D_{U}\left[D_{V}\right.$ ] is also an eigenfunction of the geodesic flow of eigenvalue $\sigma-1$, by the natural identification $K A \equiv S^{1} \times \mathbb{R}$ one can write

$$
\tilde{D}_{U}=\phi_{U} \otimes e^{\sigma t} d t \quad \text { and } \quad \tilde{D}_{V}=\phi_{V} \otimes e^{-\sigma t} d t
$$

(the parameter $t \in \mathbb{R}$ denotes the geodesic arc-length) for some distributions $\phi_{U}$, $\phi_{V} \in \mathcal{D}^{\prime}\left(S^{1}\right)$. This decomposition follows from the fact that the Lebesgue measure is the only translation invariant distribution on $\mathbb{R}$ up to constant factors. It can be checked that $\phi_{U}$ and $\phi_{V} \in \Gamma \mathcal{D}_{\sigma}^{\prime}\left(S^{1}\right)$ since $\tilde{D}_{U}$ and $\tilde{D}_{V}$ are $\Gamma$-invariant.

Conversely, given $\phi \in \Gamma \mathcal{D}_{\sigma}^{\prime}\left(S^{1}\right)$ one can check that $\phi \otimes e^{\sigma t} d t$ is a $\Gamma$-invariant distribution on $K A \equiv S^{1} \times \mathbb{R}$, hence a $\Gamma$-invariant distribution on the space of stable [unstable] horocycles, $P S L(2, \mathbb{R}) / h^{U}\left[P S L(2, \mathbb{R}) / h^{V}\right]$ (which can both be identified to $K A$ ). It follows that the distribution

$$
\phi \otimes e^{\sigma t} d t \otimes d h^{U} \quad\left[\phi \otimes e^{-\sigma t} d t \otimes d h^{V}\right],
$$

appropriately defined on $\operatorname{PSL}(2, \mathbb{R})$, is $\Gamma$-invariant, $h^{U}$-invariant $\left[h^{V}\right.$-invariant] and projects to a $h^{U}$-invariant $\left[h^{V}\right.$-invariant] distribution $D_{U}^{\phi}\left[D_{V}^{\phi}\right]$ on the manifold $\Gamma \backslash P S L(2, \mathbb{R})$. By Fubini theorem for distributions, it follows that

$$
g_{t}^{*}\left(D_{U}^{\phi}\right)=e^{(\sigma-1) t} D_{U}^{\phi}, \quad \text { and } \quad g_{t}^{*}\left(D_{V}^{\phi}\right)=e^{(1-\sigma) t} D_{V}^{\phi},
$$

that is, $D_{U}^{\phi}\left[D_{V}^{\phi}\right]$ is an eigenvector for the geodesic flow of eigenvalue $\sigma-1[1-\sigma]$. 
Cosentino (see [9], Prop. 1.2) proves the following result:

Lemma 4.2. If $\sigma(1-\sigma) \in \mathbb{R}^{+} \backslash\{1 / 4\}$ is a Casimir parameter, the map

$$
\phi \rightarrow D_{U}^{\phi}:=\phi \otimes e^{\sigma t} d t \otimes d h^{U} \quad\left[\phi \rightarrow D_{V}^{\phi}:=\phi \otimes e^{-\sigma t} d t \otimes d h^{V}\right]
$$

defines a linear isomorphism of the space ${ }^{\Gamma} \mathcal{D}_{\sigma}^{\prime}\left(S^{1}\right)$ of $\Gamma$-invariant conformal distributions of exponent $\sigma \in \mathbb{C}$ onto the spaces $\mathcal{J}_{U}^{\sigma}\left[\mathcal{J}_{V}^{\sigma}\right]$ of $U$-invariant [ $V$-invariant] distributions, which are eigenvectors of eigenvalue $\sigma-1[1-\sigma]$ with respect to the action of the geodesic flow.

Cosentino then derives a regularity result for invariant distribution from a theorem of J. P. Otal on the Poisson-Helgason transform.

Theorem 4.1. ([9], Cor. 1.4) If $\sigma(1-\sigma) \in \mathbb{R}^{+} \backslash\{1 / 4\}$ is a Casimir parameter, the space $\Gamma_{\mathcal{D}_{\sigma}^{\prime}}^{\prime}\left(S^{1}\right) \subset C^{\operatorname{Re}(\sigma)-1}\left(S^{1}\right)$, hence $\mathrm{J}^{\sigma} \subset C^{\operatorname{Re}(\sigma)-1}(\Gamma \backslash P S L(2, \mathbb{R}))$.

(Let $C^{\alpha}\left(S^{1}\right)$ be the space of all Hölder functions of exponent $\alpha \in(0,1)$. Let $C^{\alpha-1}\left(S^{1}\right)$ denote the space of all distributions in $\mathcal{D}^{\prime}\left(S^{1}\right)$ which are locally derivatives of functions in $C^{\alpha}\left(S^{1}\right)$ ).

In the exceptional case $\mu=1 / 4$, the above construction yields a 1-dimensional subspace of the 2-dimensional space of $U$-invariant [ $V$-invariant] distribution, that is, the subspace of distributional eigenvectors for the geodesic flow. Cosentino [9] proves that a second independent distribution can be constructed as follows.

Lemma 4.3. For any $\Gamma$-invariant conformal distribution $\phi \in \in^{\Gamma} \mathcal{D}_{1 / 2}^{\prime}\left(S^{1}\right)$, there exists a distribution $\phi^{\prime} \in C^{\alpha-1}\left(S^{1}\right)$ for any $\alpha<1 / 2$ such that the distribution

$$
\begin{aligned}
D_{U}^{\phi,-} & :=\left(\phi^{\prime} \otimes e^{\frac{t}{2}} d t-\phi \otimes \frac{t}{2} e^{\frac{t}{2}}\right) \otimes d h_{U}, \\
{\left[D_{V}^{\phi,+}\right.} & \left.:=\left(\phi^{\prime} \otimes e^{-\frac{t}{2}} d t+\phi \otimes \frac{t}{2} e^{-\frac{t}{2}}\right) \otimes d h_{V}\right] .
\end{aligned}
$$

is $\Gamma$-invariant and $U$-invariant [ $V$-invariant] on $P S L(2, \mathbb{R})$, hence it projects to a $U$-invariant [V-invariant] distribution on the manifold $\Gamma \backslash P S L(2, \mathbb{R})$.

The distribution $\phi^{\prime}$ is constructed in [9] as the inverse Poisson-Helgason transform (the boundary value) of the function on the Poincare disk $D$ given by the pairing of the distribution $\phi \in \in^{\Gamma} \mathcal{D}_{1 / 2}^{\prime}\left(S^{1}\right)$ with the function on $S^{1}$ defined as $P(z, \cdot)^{1 / 2} \log P(z, \cdot)$ in terms of the Poisson kernel $P$ on $D \times S^{1}$.

Let $\phi \in \Gamma_{\mathcal{D}_{1 / 2}^{\prime}}^{\prime}\left(S^{1}\right)$ be a $\Gamma$-invariant conformal distribution of exponent $1 / 2$ and let $D_{U}^{\phi,+} \in \mathcal{J}_{U}^{1 / 2}\left[D_{V}^{\phi,-} \in \mathcal{J}_{V}^{1 / 2}\right]$ be the $U$-invariant [ $V$-invariant] distribution defined as in formula (81). A direct calculations shows that formula (6) holds for the distributional vector $\left(D_{1 / 4}^{+}, D_{1 / 4}^{-}\right):=\left(D_{U}^{\phi,+}, D_{U}^{\phi,-}\right)$ [formula 25] holds for the distributional vector $\left.\left(\hat{D}_{1 / 4}^{+}, \hat{D}_{1 / 4}^{-}\right):=\left(D_{V}^{\phi,+}, D_{V}^{\phi,-}\right)\right]$. 
4.4. Proof of the correspondence (Theorem 1.9). By Theorem 1.7 there exist $V$-invariant distributions $\hat{D}_{\mu}^{ \pm}$, which are eigenvectors of eigenvalues $-(1 \pm \nu) / 2$ for the geodesic flow, such that the following identities hold on $S M$, hence on $P S L(2, \mathbb{R})$ :

$$
d t \wedge \hat{\beta}_{\mu}^{ \pm} \wedge d h^{V}=\hat{D}_{\mu}^{ \pm}
$$

(We remark that the distributions $\hat{D}_{\mu}^{ \pm}$are here identified with currents of degree 3 and dimension 0 , acting on functions, and the 1-dimensional finitely-additive measures $\beta_{\mu}^{ \pm}$to currents of degree 1 and dimension 2 , acting on 2-forms).

By Lemma 4.2, it follows that, for $\mu \neq 1 / 4$, there exist $\Gamma$-invariant conformal distributions $\phi_{\mu}^{ \pm}$of exponent $\sigma^{ \pm}:=1-(1 \pm \nu) / 2=(1 \mp \nu) / 2$ such that

$$
\hat{D}_{\mu}^{ \pm}=d t \otimes \hat{\beta}_{\mu}^{ \pm} \otimes d h^{V}=d t \wedge \hat{\beta}_{\mu}^{ \pm} \wedge d h^{V}=\phi_{\mu}^{ \pm} \otimes e^{-\frac{1 \mp \nu}{2} t} d t \otimes d h^{V} ;
$$

for $\mu=1 / 4$ there exist a $\Gamma$-invariant conformal distribution $\phi$ of exponent $1 / 2$ and a distribution $\phi^{\prime} \in C^{\alpha-1}\left(S^{1}\right)$, for all $\alpha<1 / 2$, such that

$$
\begin{aligned}
& \hat{D}_{1 / 4}^{+}=d t \otimes \hat{\beta}_{1 / 4}^{+} \otimes d h^{V}=\phi^{\prime} \otimes e^{-\frac{t}{2}} d t \otimes d h^{V}+\phi \otimes \frac{t}{2} e^{-\frac{t}{2}} d t \otimes d h^{V}, \\
& \hat{D}_{1 / 4}^{-}=d t \otimes \hat{\beta}_{1 / 4}^{-} \otimes d h^{V}=\phi \otimes e^{-\frac{t}{2}} d t \otimes d h^{V} .
\end{aligned}
$$

The statement of the theorem follows immediately.

\section{Proofs of Technical Lemmas.}

5.1. Outline of the section. In $\S \S 5.2$ we prove the key estimate on coboundaries stated in Lemma 2.4. In $\S \S 5.3$ we compute the $L^{2}$ inner products of the additive cocycles coming from a single irreducible components of the principal series and establish a non-vanishing result (Lemma 5.2). In $\$ \S 5.4$ we prove a couple of results on rotationally invariant measures (up to affine transformations) on complex Euclidean spaces (Lemma 5.7 and Lemma 5.8). These results hold for the probability distributions of additive cocycles coming from irreducible components of the principal series by the above-mentioned non-vanishing result (Lemma 5.2 of $\S \S 5.3$ and are motivated by the conditional results on the existence of limit distributions (Corollary 1.4 and Corollary 1.5 in Section 3 ).

5.2. Estimates on coboundaries. We prove below the key Lemma 2.4 which is part of the proof of Theorem 2.1 in Section 2. The subspace $\operatorname{Ker}\left(\mathcal{J}_{U}^{-s}\left(H_{\mu}\right)\right)$ is closed in $W^{s}\left(H_{\mu}\right)$ and we introduce the orthogonal projection

$$
P_{\mu}^{s}: W^{s}\left(H_{\mu}\right) \rightarrow \operatorname{Ker}\left(\mathcal{J}_{U}^{-s}\left(H_{\mu}\right)\right) .
$$

As above let $\nu=\sqrt{1-4 \mu}$. Let $s_{\mu}:=(1+|\operatorname{Re} \nu|) / 2$, that is, $s_{\mu}=1 / 2(1+\nu)$ for $0<\mu<1 / 4 ; s_{\mu}=1 / 2$ for $\mu \geq 1 / 4$ and $s_{\mu}=n$ for $\mu=-n^{2}+n$.

Lemma 5.1. For any non-trivial irreducible unitary representation of Casimir parameter $\mu \in \mathbb{R} \backslash\{0\}$ and for any $s \geq r>s_{\mu}$ there exists a constant $C_{r, s}(\mu)>0$ such that the following holds. For any $f \in W^{s}\left(H_{\mu}\right)$ we have

$$
\left\|P_{\mu}^{s} f\right\|_{r} \leq C_{r, s}(\mu)\|f\|_{r} .
$$


For any $\mu_{0}>1 / 4$ and for all $s \geq r>1 / 2$, there exists a constant $C_{r, s}\left(\mu_{0}\right)>0$ such that, for all $\mu \geq \mu_{0}$ and for all $f \in W^{s}\left(H_{\mu}\right)$,

$$
\left\|P_{\mu}^{s} f\right\|_{r} \leq C_{r, s}\left(\mu_{0}\right)\|f\|_{r} .
$$

Proof. Consider first the case of the principal and the complementary series. Let $\chi_{\mu}^{ \pm}(s) \in\left(\operatorname{Ker}\left(\mathcal{J}_{U}^{-s}\left(H_{\mu}\right)\right)^{s}\right)^{\perp} \subset W^{s}\left(H_{\mu}\right)$ be functions such that

$$
\begin{aligned}
& D_{\mu}^{+}\left(\chi_{\mu}^{+}(s)\right)=D_{\mu}^{-}\left(\chi_{\mu}^{-}(s)\right)=1, \\
& D_{\mu}^{+}\left(\chi_{\mu}^{-}(s)\right)=D_{\mu}^{-}\left(\chi_{\mu}^{+}(s)\right)=0 .
\end{aligned}
$$

Such functions exist since $\left\{D_{\mu}^{+}, D_{\mu}^{-}\right\}$is a basis for $\mathcal{J}_{U}^{-s}\left(H_{\mu}\right)$ and, by definition of the space $\operatorname{Ker}\left(\mathcal{J}_{U}^{-s}\left(H_{\mu}\right)\right)$, the functionals $D_{\mu}^{ \pm}$induce linearly independent functionals on the 2-dimensional quotient space $W^{s}\left(H_{\mu}\right) / \operatorname{Ker}\left(\mathcal{J}_{U}^{-s}\left(H_{\mu}\right)\right)$. In fact, take a pair of functions $\left\{\chi^{+}, \chi^{-}\right\} \subset W^{s}\left(H_{\mu}\right)$ which project onto a dual basis of $\left\{D_{\mu}^{+}, D_{\mu}^{-}\right\}$under the projection $W^{s}\left(H_{\mu}\right) \rightarrow W^{s}\left(H_{\mu}\right) / \operatorname{Ker}\left(\mathcal{J}_{U}^{-s}\left(H_{\mu}\right)\right)$ and define $\chi_{\mu}^{ \pm}(s)$ as the orthogonal projections onto $\operatorname{Ker}\left(\mathcal{J}_{U}^{-s}\left(H_{\mu}\right)\right)^{\perp} \subset W^{s}\left(H_{\mu}\right)$ of $\chi^{ \pm}$ respectively. By construction we have

$$
P_{\mu}^{s} f=f-D_{\mu}^{+}(f) \chi_{\mu}^{+}(s)-D_{\mu}^{-}(f) \chi_{\mu}^{-}(s) .
$$

Indeed, by formula (83) the right hand side of the formula clearly belongs to the kernel of both $D_{\mu}^{ \pm}$and, besides, if $P_{\mu}^{s} f$ is defined by (84), then we clearly have $f-P_{\mu}^{s} f \perp \operatorname{Ker}\left(\mathcal{J}_{U}^{-s}\left(H_{\mu}\right)\right)$. It follows that for any $s_{\mu}<r \leq s$ and for any function $f \in W^{s}\left(H_{\mu}\right)$ the following bound holds:

$$
\left\|P_{\mu}^{s} f\right\|_{r} \leq\|f\|_{r}+\left\|\chi_{\mu}^{+}(s)\right\|_{r}\left\|D_{\mu}^{+}\right\|_{-r}\|f\|_{r}+\left\|\chi_{\mu}^{-}(s)\right\|_{r}\left\|D_{\mu}^{-}\right\|_{-r}\|f\|_{r},
$$

and, since $\left\|\chi_{\mu}^{ \pm}(s)\right\|_{r}<+\infty$ for any $r \leq s$ and $D_{\mu}^{ \pm} \in W^{-r}\left(H_{\mu}\right)$ for $r>s_{\mu}$ ([12], Theorem 1.1 or Theorem 3.2), the proof is complete for the principal and the complementary series.

The proof for the discrete series is similar, in fact simpler since in each irreducible component of the discrete series there is only one invariant distribution $\mathcal{D}_{\mu}$ (up to constant factors), and we only need one smooth function $\chi_{\mu}(s)$ such that $D_{\mu}\left(\chi_{\mu}(s)\right)=1$ and $\chi_{\mu}(s) \perp \operatorname{Ker}\left(\mathcal{J}_{U}^{-s}\left(H_{\mu}\right)\right)$. We then write $P_{\mu}^{s} f=$ $f-D_{\mu}(f) \chi_{\mu}(s)$, and the rest of the proof is identical.

It remains to be proved that the family of projection operators $P_{\mu}^{s}$ extends to a uniformly bounded family of operators on $W^{r}\left(H_{\mu}\right)$ for $\mu \geq \mu_{0}>1 / 4$.

As observed in the proof of Lemma 5.1 in [12], if the Casimir parameter $\mu \geq$ $\mu_{0}>1 / 4$, for any $r>1$, the distorsion in $W^{-r}(S M)$ of the system of distributions $\left\{D_{\mu}^{+}, D_{\mu}^{-}\right\}$stays bounded above (in other terms, the angle in $W^{-r}(S M)$ between $D_{\mu}^{+}$and $D_{\mu}^{-}$stays bounded below), and in fact this bound is uniform with respect to $r>1$. Hence there exists a constant $C_{r, s}\left(\mu_{0}\right)>0$ such that, for all $r>1$,

$$
\left\|\chi_{\mu}^{ \pm}(s)\right\|_{r} \leq C_{r, s}\left(\mu_{0}\right) /\left\|D_{\mu}^{ \pm}\right\|_{-r} .
$$

The above argument then yields the uniform bound on the norm of the operators $P_{\mu}^{s}: W^{r}\left(H_{\mu}\right) \rightarrow \operatorname{Ker}\left(\mathcal{J}_{U}^{-r}\left(H_{\mu}\right)\right)$ for $\mu \geq \mu_{0}>1 / 4$. 
Let us recall that we have defined $\mathcal{R}^{-s}: \Omega_{1}^{-s}(S M) \rightarrow \mathcal{B}_{U}^{-s}(S M)^{\perp}$ as the orthogonal projection onto the orthogonal complement of the subspace of basic currents for the stable horocycle. For any irreducible component $H_{\mu} \subset L^{2}(S M)$, of Casimir parameter $\mu \in \mathbb{R} \backslash\{0\}$, we have defined $\Pi_{\mu}^{-s}: \Omega_{1}^{-s}(S M) \rightarrow \Omega_{1}^{-s}\left(H_{\mu}\right)$ as the orthogonal projection onto the corresponding Sobolev space. We have then defined $\mathcal{R}_{\mu}^{-s}=\Pi_{\mu}^{-s} \circ \mathcal{R}^{-s}: \Omega_{1}^{-s}(S M) \rightarrow \mathcal{B}_{U}^{-s}\left(H_{\mu}\right)^{\perp}$ as the orthogonal projection onto the orthogonal complement of the subspace of basic currents in $\Omega_{1}^{-s}\left(H_{\mu}\right)$.

Proof of Lemma 2.4 The argument is similar to the proof Lemma 5.5 in [12].

By Hilbert space theory, any current $\mathcal{R}^{-s} \in \mathcal{B}_{U}^{-s}(S M)^{\perp} \subset \Omega_{1}^{-s}(S M)$ (orthogonal to the subspace of basic currents) has the following property:

$$
\mathcal{R}^{-s}(\lambda)=0, \quad \text { for any } \lambda \in\left[\operatorname{Ker}\left(\mathcal{B}_{U}^{-s}\left(H_{\mu}\right)\right)\right]^{\perp} .
$$

In fact, the Hilbert space $\Omega_{1}^{-s}\left(H_{\mu}\right)$ is defined as the dual space $\Omega_{1}^{s}\left(H_{\mu}\right)^{*}$, which in turn is isomorphic to $\Omega_{1}^{s}\left(H_{\mu}\right)$. Thus we have

$$
\operatorname{Ker}\left(S^{\perp}\right)=[\operatorname{Ker}(S)]^{\perp}, \quad \text { for any subspace } S \subset \Omega_{1}^{-s}\left(H_{\mu}\right) .
$$

It follows that $\operatorname{Ker}\left[\mathcal{B}_{U}^{-s}\left(H_{\mu}\right)^{\perp}\right]=\left[\operatorname{Ker}\left(\mathcal{B}_{U}^{-s}\left(H_{\mu}\right)\right)\right]^{\perp}$, hence, in particular,

$$
\mathcal{R}^{-s} \in \mathcal{B}_{U}^{-s}\left(H_{\mu}\right)^{\perp}=\operatorname{Ker}\left[\operatorname{Ker}\left(\mathcal{B}_{U}^{-s}\left(H_{\mu}\right)\right)\right]^{\perp} .
$$

By the characterization of basic currents for the stable horocycle flow given by Lemma 2.1, the kernel $\operatorname{Ker}\left(\mathcal{B}_{U}^{-s}\left(H_{\mu}\right)\right)$ can be described as follows:

$$
\operatorname{Ker}\left(\mathcal{B}_{U}^{-s}\left(H_{\mu}\right)\right):=\left\{\lambda=\lambda_{X} \hat{X}+\lambda_{U} \hat{U}+\lambda_{V} \hat{V} \mid \lambda_{U} \in \operatorname{Ker}\left(\mathcal{J}_{U}^{-s}\left(H_{\mu}\right)\right)\right\} .
$$

By the definition of the Hilbert structure of the space $\Omega_{1}^{s}(S M) \equiv W^{s}(S M)^{3}$, it follows that the orthogonal projection $\Pi_{\mu}^{s}: \Omega_{1}^{s}\left(H_{\mu}\right) \rightarrow \operatorname{Ker}\left(\mathcal{B}_{U}^{-s}\left(H_{\mu}\right)\right)$ can be written in terms of the orthogonal projection $P_{\mu}^{s}: W^{s}\left(H_{\mu}\right) \rightarrow \operatorname{Ker}\left(\mathcal{J}_{U}^{-s}\left(H_{\mu}\right)\right)$ :

$$
\Pi_{\mu}^{s}(\lambda)=\Pi_{\mu}^{s}\left(\lambda_{X} \hat{X}+\lambda_{U} \hat{U}+\lambda_{V} \hat{V}\right)=\lambda_{X} \hat{X}+P_{\mu}^{s}\left(\lambda_{U}\right) \hat{U}+\lambda_{V} \hat{V} .
$$

For any $\lambda \in \Omega_{1}^{s}\left(H_{\mu}\right)$, since by definition $\mathcal{B}^{-s}(\gamma) \circ \Pi_{\mu}^{s}=0$, by definition of $\mathcal{R}_{\mu}^{-s}(\gamma)$ and by the vanishing established in formula $[85)$,

$$
\mathcal{R}_{\mu}^{-s}(\gamma)(\lambda)=\left(\mathcal{R}^{-s}(\gamma) \circ \Pi_{\mu}^{s}\right)(\lambda)=\gamma\left(\Pi_{\mu}^{s}(\lambda)\right) .
$$

By [12], Theorem 4.1, since $P_{\mu}^{s}\left(\lambda_{U}\right) \in \operatorname{Ker}\left(\mathcal{J}_{U}^{-s}\left(H_{\mu}\right)\right)$, there exists a unique solution $f_{\lambda} \in W^{r}\left(H_{\mu}\right)$ (for all $r<s-1$ ) of the cohomological equation

$$
U f_{\lambda}=P_{\mu}^{s}\left(\lambda_{U}\right) \text {. }
$$

Moreover, for any $s_{\mu}<r \leq s$ and any $\rho<r-1$, we have $f_{\lambda} \in W^{\rho}\left(H_{\mu}\right)$, and there exists a constant $C_{\rho, r}$, depending only on $\rho, r$ such that we have

$$
\left\|f_{\lambda}\right\|_{\rho} \leq C_{\rho, r}\left\|P_{\mu}^{s}\left(\lambda_{U}\right)\right\|_{r} .
$$

Thus by Lemma 5.1 we can conclude that for all Casimir parameters $\mu \neq 0$, for any $s_{\mu}<r \leq s$ and any $\rho<r-1$, there exists $C_{s, \rho, r}(\mu)>0$ such that

$$
\left\|f_{\lambda}\right\|_{\rho} \leq C_{s, \rho, r}(\mu)\left\|\lambda_{U}\right\|_{r} \leq C_{s, \rho, r}(\mu)\|\lambda\|_{r}
$$


and that for any $\mu_{0}>1 / 4$ and for $1 / 2<r \leq s$ and any $\rho<r-1$ there exists a constant $C_{s, \rho, r}\left(\mu_{0}\right)>0$ such that, for all $\mu \geq \mu_{0}$,

$$
\left\|f_{\lambda}\right\|_{\rho} \leq C_{s, \rho, r}\left\|\lambda_{U}\right\|_{r} \leq C_{s, \rho, r}\left(\mu_{0}\right)\|\lambda\|_{r} .
$$

Let $x, y \in S M$ be the endpoints of the arc $\gamma$. By the formula

$$
d f_{\lambda}=X f_{\lambda} \hat{X}+U f_{\lambda} \hat{U}+V f_{\lambda} \hat{V}
$$

it follows that the following identity holds:

$$
\gamma\left(\Pi_{\mu}^{s}(\lambda)\right)=\gamma\left[\left(\lambda_{X}-X f_{\lambda}\right) \hat{X}+\left(\lambda_{V}-V f_{\lambda}\right) \hat{V}\right]+f_{\lambda}(y)-f_{\lambda}(x) .
$$

The above identity yields the following estimate:

$$
\left|\gamma\left(\Pi_{\mu}^{s}(\lambda)\right)\right| \leq\left(\left\|\lambda_{X}\right\|_{\infty}+\left\|\lambda_{V}\right\|_{\infty}+\left\|d f_{\lambda}\right\|_{\infty}\right)\left(\int_{\gamma}|\hat{X}|+\int_{\gamma}|\hat{V}|\right)+2\left\|f_{\lambda}\right\|_{\infty} .
$$

By the Sobolev Embedding Theorem, for any $\rho \in(5 / 2, s-1)$, there exists a positive constant $C_{\rho}(M)$ depending only on $M$ such that

$$
\left\|f_{\lambda}\right\|_{\infty}+\left\|d f_{\lambda}\right\|_{\infty} \leq C_{\rho}(M)\left\|f_{\lambda}\right\|_{\rho}
$$

We thus obtain that for any $s \geq r>7 / 2$ and for all Casimir parameters $\mu>0$ there esists a constant $C_{r, s}(\mu)>0$ such that, for all $\lambda \in \Omega_{1}^{s}\left(H_{\mu}\right)$,

$$
\left|\mathcal{R}_{\mu}^{-s}(\gamma)(\lambda)\right|=\left|\gamma\left(\Pi_{\mu}^{s}(\lambda)\right)\right| \leq C_{r, s}(\mu)\left(1+\int_{\gamma}|\hat{X}|+\int_{\gamma}|\hat{V}|\right)\|\lambda\|_{r}
$$

for any $s \geq r>7 / 2$ and for any $\mu_{0}>1 / 4$, there exists a constant $C_{r, s}\left(\mu_{0}\right)>0$ such that, for all $\mu \geq \mu_{0}$ and for all $\lambda \in \Omega_{1}^{s}(S M)$,

$$
\left|\mathcal{R}_{\mu}^{-s}(\gamma)(\lambda)\right|=\left|\gamma\left(\Pi_{\mu}^{s}(\lambda)\right)\right| \leq C_{r, s}\left(\mu_{0}\right)\left(1+\int_{\gamma}|\hat{X}|+\int_{\gamma}|\hat{V}|\right)\|\lambda\|_{r}
$$

Since $\Omega_{1}^{s}\left(H_{\mu}\right)$ is dense in $\Omega_{1}^{r}\left(H_{\mu}\right)$ for $s \geq r$, it follows that the distribution $\mathcal{R}_{\mu}^{-s}(\gamma) \in \Omega_{1}^{-s}\left(H_{\mu}\right)$ has a unique continuous extension $\mathcal{R}_{\mu}^{-s,-r}(\gamma)$ to the space $\Omega_{1}^{r}\left(H_{\mu}\right)$ such that

$$
\left\|\mathcal{R}_{\mu}^{-s,-r}(\gamma)(\lambda)\right\|_{-r} \leq C_{r, s}(\mu)\left(1+\int_{\gamma}|\hat{X}|+\int_{\gamma}|\hat{V}|\right)
$$

for all $\mu_{0}>1 / 4$, there exists a constant $C_{r, s}\left(\mu_{0}\right)>0$ such that for all $\mu \geq \mu_{0}$,

$$
\left\|\mathcal{R}_{\mu}^{-s,-r}(\gamma)(\lambda)\right\|_{-r} \leq C_{r, s}\left(\mu_{0}\right)\left(1+\int_{\gamma}|\hat{X}|+\int_{\gamma}|\hat{V}|\right) .
$$

Since $M$ is a compact hyperbolic surface, the Casimir operator of the standard unitary representation of $S L(2, \mathbb{R})$ on $L^{2}(S M)$ has discrete spectrum, hence for any $s \geq r>7 / 2$ there is a constant $C_{r, s}>0$ (depending only on $r, s$ ) such that, for all Casimir parameters $\mu>0$ and for every rectifiable arc $\gamma$ in $S M$, we have

$$
\left\|\mathcal{R}_{\mu}^{-s,-r}(\gamma)\right\|_{-r} \leq C_{r, s}\left(1+\int_{\gamma}|\hat{X}|+\int_{\gamma}|\hat{V}|\right) .
$$

Thus Lemma2.4 is completely proved. 
5.3. Inner products of cocycles. Cocycles which belong to different irreducible components are orthogonal. We compute below the inner product of the two complex-conjugate coycles which belong to a given component principal series type and prove that it is non-zero.

Lemma 5.2. For any $\mu>1 / 4$ and any $T \in \mathbb{R} \backslash\{0\}$, the $L^{2}$ inner product

$$
<\beta_{\mu}^{+}(\cdot, T), \beta_{\mu}^{-}(\cdot, T)>\neq 0 .
$$

Proof. The computation proceeds as follows. Let $\left\{f^{+}, f^{-}\right\} \in C^{\infty}\left(H_{\mu}\right)$ be a pair of functions dual to the basis $\left\{D_{\mu}^{+}, D_{\mu}^{-}\right\}$of invariant distributions, that is,

$$
D_{\mu}^{+}\left(f^{+}\right)=D_{\mu}^{-}\left(f^{-}\right) \neq 0, \quad D_{\mu}^{+}\left(f^{-}\right)=D_{\mu}^{-}\left(f^{+}\right)=0 .
$$

It follows from Corollary 3.2 that

$$
\begin{aligned}
& e^{-(1-\nu) t}<\int_{0}^{T e^{t}} f^{+} \circ h_{\sigma}^{U} d \sigma, \int_{0}^{T e^{t}} f^{-} \circ h_{\tau}^{U} d \tau> \\
& -D_{\mu}^{+}\left(f^{+}\right) \overline{D_{\mu}^{-}\left(f^{-}\right)}<\beta_{\mu}^{+}(\cdot, T), \beta_{\mu}^{-}(\cdot, T)>=O\left(\exp \left(-\epsilon_{\mu} t\right)\right) .
\end{aligned}
$$

Our goal is therefore to compute the asymptotics of the normalized inner product

$$
\mathcal{E}_{T, t}\left(f^{+}, f^{-}\right):=e^{-(1-\nu) t} \int_{0}^{T e^{t}} \int_{0}^{T e^{t}}<f^{+} \circ h_{\sigma}^{U}, f^{-} \circ h_{\tau}^{U}>d \sigma d \tau .
$$

The above computation can be performed explicitly in the standard model for representations of the principal series on the Hilbert space $L^{2}(\mathbb{R}, d x)$.

In this model, the horocycle flow is represented by the group of translations, hence its infinitesimal generator is the operator $d / d x$. In general, the action of $S L(2, \mathbb{R})$ on $L^{2}(\mathbb{R}, d x)$ for the irreducible representation $\pi_{\mu}$ of the principal series is given by the formula:

$$
\pi_{\mu}\left[\left(\begin{array}{ll}
a & b \\
c & d
\end{array}\right)\right] f(x):=|c x+d|^{-(1+\nu)} f\left(\frac{a x+b}{c x+d}\right) .
$$

Hence the derived representation $d \pi_{\mu}$ of the Lie algebra $\mathfrak{s l}(2, \mathbb{R})$ of $S L(2, \mathbb{R})$ is described by the following formulas:

$$
\begin{aligned}
& d \pi_{\mu}(U)=\frac{d}{d x} \\
& d \pi_{\mu}(V)=-x^{2} \frac{d}{d x}-(1+\nu) x \\
& d \pi_{\mu}(X)=x \frac{d}{d x}+\frac{1+\nu}{2} .
\end{aligned}
$$

>From the above formulas one can also deduce the formula for the representation of the generator $\Theta$ of the circle action on the unit tangent bundle. In fact, since $\Theta=U-V$, we have

$$
d \pi_{\mu}(\Theta)=\left(1+x^{2}\right) \frac{d}{d x}+(1+\nu) x .
$$


A calculus exercise shows that, up to normalization, the unique $\Theta$-invariant function $u_{0} \in C^{\infty}\left(H_{\mu}\right)$ is given in the representation model by the formula:

$$
u_{0}(x):=\frac{1}{\left(1+x^{2}\right)^{\frac{1+\nu}{2}}}, \quad x \in \mathbb{R} .
$$

It follows from the construction of the $U$-invariant distributions in [12], §3, that the following holds:

$$
D_{\mu}^{+}\left(u_{0}\right) \neq 0 \text {. }
$$

Given any function $f^{-}$such that $D_{\mu}^{+}\left(f^{-}\right)=0$ and $D_{\mu}^{-}\left(f^{-}\right) \neq 0$, we can therefore choose

$$
f^{+}:=u_{0}-\frac{D_{\mu}^{-}\left(u_{0}\right)}{D_{\mu}^{-}\left(f^{-}\right)} f^{-} .
$$

We will choose $f^{-}$to be represented by any function in $C_{0}^{\infty}(\mathbb{R}) \subset L^{2}(\mathbb{R}, d x)$ with non-zero integral over the real line. In order to justify this choice we remark that the $U$-invariant distribution $D_{\mu}^{-}$is an extension to $C^{\infty}\left(H_{\mu}\right)$ of the distribution $\mathcal{A} \in \mathcal{D}^{\prime}(\mathbb{R})$ given by the average over the real line, while the $U$-invariant distribution $D_{\mu}^{+}$vanishes identically on the (non-dense!) subspace $C_{0}^{\infty}(\mathbb{R}) \subset C^{\infty}\left(H_{\mu}\right)$. In fact, since $D_{\mu}^{ \pm}$are $U$-invariant and the average $\mathcal{A}$ is $d \pi_{\mu}(U)$-invariant, there exists constants $c^{ \pm} \in \mathbb{R}$ such that

$$
D_{\mu}^{ \pm}=c^{ \pm} \mathcal{A} \quad \text { on } C_{0}^{\infty}(\mathbb{R}) \subset C^{\infty}\left(H_{\mu}\right) .
$$

However, the following formulas hold in $\mathcal{D}^{\prime}(\mathbb{R})$ :

$$
d \pi_{\mu}(X) \mathcal{A}=-\frac{1-\nu}{2} \mathcal{A} .
$$

It follows that $c^{+}=0$, since $d \pi_{\mu}(X) D_{\mu}^{+}=-\frac{1+\nu}{2} D_{\mu}^{+}$, and that $c^{-} \neq 0$, that is, $D_{\mu}^{-}$is, up to a non-zero multiplicative constant, an extension of the distribution $\mathcal{A}$ to $C^{\infty}\left(H_{\mu}\right)$. Hence for any function $f^{-}$represented by a smooth real-valued function with compact support and non-zero integral,

$$
D_{\mu}^{+}\left(f^{-}\right)=0 \quad \text { and } \quad D_{\mu}^{-}\left(f^{-}\right)=c^{-} \mathcal{A}\left(f^{-}\right) \neq 0 .
$$

We remark that by Corollary 3.2 the normalized inner product

$$
\varepsilon_{T, t}\left(f^{-}, f^{-}\right):=e^{-(1-\nu) t}\left\|\int_{0}^{T e^{t}} f^{-} \circ h_{\sigma}^{U} d \sigma\right\|^{2}
$$

is given asymptotically by the following formula:

$$
\varepsilon_{T, t}\left(f^{-}, f^{-}\right)-e^{\nu t}\left|D_{\mu}^{-}\left(f^{-}\right)\right|^{2}\left\|\beta_{\mu}^{-}(\cdot, T)\right\|^{2}=O\left(\exp \left(-\epsilon_{\mu} t\right)\right) .
$$

Our task is therefore reduced to estimate the integral

$$
\int_{0}^{T e^{t}} \int_{0}^{T e^{t}} \int_{\mathbb{R}} \frac{f^{-}(x+\tau)}{\left(1+(x+\sigma)^{2}\right)^{\frac{1+\nu}{2}}} d x d \sigma d \tau .
$$

By Fubini's and change of variables theorems, it is enough to compute the integral

$$
I_{T}(x):=\int_{0}^{T} \int_{0}^{T} \frac{1}{\left(1+(x+\sigma-\tau)^{2}\right)^{\frac{1+\nu}{2}}} d x .
$$


By explicit integration we obtain that as $T \rightarrow+\infty$ the function

$$
T^{-(1-\nu)} I_{T}(x)-T^{\nu} \int_{-T}^{T} \frac{d u}{\left(1+u^{2}\right)^{\frac{1+\nu}{2}}}-\frac{2}{1-\nu} \rightarrow 0
$$

uniformly on compact intervals. By integration by parts the integral

$$
J_{T}:=\int_{-T}^{T} \frac{d u}{\left(1+u^{2}\right)^{\frac{1+\nu}{2}}}
$$

satisfies the following formula:

$$
J_{T}=\frac{2 T}{\left(1+T^{2}\right)^{\frac{1+\nu}{2}}}+(1+\nu) J_{T}-(1+\nu) \int_{-T}^{T} \frac{d u}{\left(1+u^{2}\right)^{\frac{3+\nu}{2}}} .
$$

It follows immediately that

$$
J_{T}=-\frac{1}{\nu} \frac{2 T}{\left(1+T^{2}\right)^{\frac{1+\nu}{2}}}+\frac{1+\nu}{\nu} \int_{-T}^{T} \frac{d u}{\left(1+u^{2}\right)^{\frac{3+\nu}{2}}} .
$$

Since the convergence in formula (98) is uniform on compact sets and the improper integral

$$
I_{\nu}:=\int_{-\infty}^{\infty} \frac{d u}{\left(1+u^{2}\right)^{\frac{3+\nu}{2}}}=\lim _{T \rightarrow+\infty} \int_{-T}^{T} \frac{d u}{\left(1+u^{2}\right)^{\frac{3+\nu}{2}}}
$$

is absolutely convergent, we obtain that the normalized integral

$$
\varepsilon_{T, t}\left(u_{0}, f^{-}\right):=e^{-(1-\nu) t}<\int_{0}^{T e^{t}} u_{0} \circ h_{\sigma}^{U} d \sigma, \int_{0}^{T e^{t}} f^{-} \circ h_{\tau}^{U} d \tau>
$$

is given asymptotically (as $t \rightarrow+\infty$ ) by the following formula:

$$
\mathcal{E}_{T, t}\left(u_{0}, f^{-}\right)+\frac{2(1-2 \nu)}{\nu(1-\nu)} T^{1-\nu} D_{\mu}^{-}\left(f^{-}\right)-\frac{1+\nu}{\nu} I_{\nu} T D_{\mu}^{-}\left(f^{-}\right) e^{\nu t} \rightarrow 0
$$

By definition we have

$$
\varepsilon_{T, t}\left(f^{+}, f^{-}\right)=\varepsilon_{T, t}\left(u_{0}, f^{-}\right)-\frac{D_{\mu}^{-}\left(u_{0}\right)}{D_{\mu}^{-}\left(f^{-}\right)} \varepsilon_{T, t}\left(f^{-}, f^{-}\right)
$$

Since the normalized inner product $\varepsilon_{T, t}\left(f^{+}, f^{-}\right)$converges, by formulas (97) and (100) it follows that

$$
\begin{aligned}
\mathcal{E}_{T, t}\left(f^{+}, f^{-}\right) & \rightarrow-\frac{2(1-2 \nu)}{\nu(1-\nu)} T^{1-\nu} D_{\mu}^{-}\left(f^{-}\right) ; \\
e^{-\nu t} \mathcal{E}_{T, t}\left(f^{-}, f^{-}\right) & \rightarrow \frac{1+\nu}{\nu} I_{\nu} T \frac{D_{\mu}^{-}\left(f^{-}\right)^{2}}{D_{\mu}^{-}\left(u_{0}\right)} .
\end{aligned}
$$

We have thus proved the lemma and in addition we obtain the formulas:

$$
\begin{aligned}
<\beta_{\mu}^{+}(\cdot, T), \beta_{\mu}^{-}(\cdot, T)> & =-\frac{2(1-2 \nu)}{\nu(1-\nu)} \frac{T^{1-\nu}}{D_{\mu}^{-}\left(u_{0}\right)} \\
\left\|\beta_{\mu}^{ \pm}(\cdot, T)\right\|^{2} & =\frac{1+\nu}{\nu} \frac{I_{\nu} T}{D_{\mu}^{-}\left(u_{0}\right)} .
\end{aligned}
$$


5.4. Rotationally symmetric measures. We prove below some elementary results about compactly supported measures on the complex plane and on higher dimensional cartesian products of the complex plane. These results characterize probability distributions with rotational symmetries up to an affine change of coordinates.

Lemma 5.3. Let $\mu$ be a compactly supported Borel measure on the complex plane. If for any $a<b \in \mathbb{R}$ there exists a constant $m(a, b)$ such that, for all $v \in \mathbb{C} \backslash\{0\}$,

$$
\mu\left\{z \in \mathbb{C} \mid a \leq \frac{\operatorname{Re}(z v)}{|v|}<b\right\}=m(a, b),
$$

then the measure $\mu$ on $\mathbb{C}$ is rotationally invariant.

Proof. It follows from the assumption that the probability distribution with respect to the measure $\mu$ on $\mathbb{C}$ of the function

$$
\operatorname{Re}\left(e^{i \theta} z\right)=\frac{e^{i \theta} z+e^{-i \theta} \bar{z}}{2},
$$

does not depend on $\theta \in \mathbb{R}$, hence for every $k \in \mathbb{Z}$ the integral

$$
\int_{\mathbb{C}}\left(e^{i \theta} z+e^{-i \theta} \bar{z}\right)^{k} d \mu
$$

does not depend on $\theta \in \mathbb{R}$ as well. It follows that

$$
\int_{\mathbb{C}} z^{r} \bar{z}^{s} d \mu=0 \quad \text { for all } r, s \in \mathbb{N}, r-s \neq 0 .
$$

Let $R_{\theta}$ be the rotation of angle $\theta \in \mathbb{R}$ on $\mathbb{C}$. For any real analytic function $f$ on $\mathbb{C}$, we have by power series expansion

$$
\int_{\mathbb{C}} f \circ R_{\theta} d \mu=\sum_{r \in \mathbb{N}} \frac{1}{(r !)^{2}} \frac{\partial^{2} f}{\partial z^{r} \partial \bar{z}^{r}}(0) \int_{\mathbb{C}}|z|^{2 r}=\int_{\mathbb{C}} f d \mu .
$$

Since $\mu$ has compact support and polynomials are dense in the uniform topology on any compact subset of the complex plane, the result follows.

For any $A, B \in \mathbb{R}^{+}$, let $T_{A, B}: \mathbb{C} \rightarrow \mathbb{C}$ the affine map defined as follows:

$$
T_{A, B}(x, y)=(x / A, y / B), \quad \text { for all }(x, y) \in \mathbb{C} \equiv \mathbb{R}^{2} .
$$

Lemma 5.4. Let $\mu$ be a compactly supported Borel probability measure on the complex plane. Assume that there exist $(A, B) \in\left(\mathbb{R}^{+}\right)^{2}$ such that for any $a, b \in \mathbb{R}$ there exists a constant $m(a, b)$ such that, for all $\theta \in \mathbb{R}$,

$$
\mu\left\{z \in \mathbb{C} \mid a \leq \frac{\operatorname{Re}\left(e^{i \theta} z\right)}{\left(A^{2} \cos ^{2} \theta+B^{2} \sin ^{2} \theta\right)^{1 / 2}}<b\right\}=m_{A, B}(a, b) .
$$

Then the measure $\mu$ has the following form: there exists a rotationally invariant measure $\rho$ on $\mathbb{C}$ such that

$$
\mu=\left(T_{A, B}^{-1}\right)_{*}(\rho) .
$$


Proof. We claim that, by Lemma 5.3, the measure $\rho:=\left(T_{A, B}\right)_{*} \mu$ is rotationally invariant. In fact, for any $\theta \in \mathbb{R}$, let $v_{A, B}(\theta) \in \mathbb{C}$ be the unit complex number defined as follows:

$$
v_{A, B}(\theta):=\frac{(A \cos \theta, B \sin \theta)}{\left(A^{2} \sin \theta+B^{2} \cos ^{2} \theta\right)^{1 / 2}} .
$$

A straightforward calculation yields that, for any $a<b$ and for all $\theta \in \mathbb{R}$,

$$
\begin{aligned}
& \rho\left\{z \in \mathbb{C} \mid a \leq \frac{\operatorname{Re}\left[z v_{A, B}(\theta)\right]}{\left|v_{A, B}(\theta)\right|}<b\right\} \\
& =\mu\left\{z \in \mathbb{C} \mid a \leq \frac{\operatorname{Re}\left(e^{i \theta} z\right)}{\left(A^{2} \cos ^{2} \theta+B^{2} \sin ^{2} \theta\right)^{1 / 2}}<b\right\} .
\end{aligned}
$$

Since the family $\left\{v_{A, B}(\theta) \mid \theta \in \mathbb{R}\right\}$ coincides with $\{v \in \mathbb{C}|| v \mid=1\}$, it follows that

$$
\rho\left\{z \in \mathbb{C} \mid a \leq \frac{\operatorname{Re}(z v)}{|v|}<b\right\}=m_{A, B}(a, b),
$$

hence $\rho$ is rotationally invariant by Lemma 5.3 , as claimed.

Lemma 5.5. Let $\beta:(X, \mu) \rightarrow \mathbb{C}$ be a bounded measurable function on the probability space $(X, \mu)$. The family of real-valued functions

$$
\left\{\frac{\operatorname{Re}\left(e^{i \theta} \beta\right)}{\left\|\operatorname{Re}\left(e^{i \theta} \beta\right)\right\|} \mid \theta \in \mathbb{R}\right\}
$$

has a constant probability distribution if and only if there exists an affine map $T: \mathbb{C} \rightarrow \mathbb{C}$ such that the function $T \circ \beta: X \rightarrow \mathbb{C}$ has a rotationally invariant probability distribution.

Proof. Up to composition with a rotation of the complex plane it is possible to assume that

$$
\int_{X}(\operatorname{Re} \beta)(\operatorname{Im} \beta) d \mu=\frac{1}{2} \operatorname{Im}\left(\int_{X} \beta^{2} d \mu\right)=0
$$

Under that assumption, it follows that

$$
\left\|\operatorname{Re}\left(e^{i \theta} \beta\right)\right\|^{2}=\|\operatorname{Re} \beta\|^{2} \cos ^{2} \theta+\|\operatorname{Im} \beta\|^{2} \sin ^{2} \theta .
$$

Thus the statement follows from Lemma 5.4

The above results generalize to functions with values in higher dimensional complex spaces. In fact, the following holds:

Lemma 5.6. Let $\mu$ be a compactly supported Borel probability measure on $\mathbb{C}^{n}$. Assume that for any $a, b \in \mathbb{R}$ there exists a constant $m(a, b)$ such that, for all $v \in \mathbb{C}^{n} \backslash\{0\}$,

$$
\mu\left\{z \in \mathbb{C} \mid a \leq \frac{\operatorname{Re}(z \cdot v)}{|v|}<b\right\}=m(a, b),
$$

then $\mu$ is rotationally invariant, that is, it is invariant under the action of the orthogonal group $S O(2 n, \mathbb{R})$ on $\mathbb{C}^{n} \equiv \mathbb{R}^{2 n}$. 
Proof. We claim that there exists a compactly supported probability measure $m$ on the real line such that the constants $m(a, b)=m\{x \in \mathbb{R} \mid a \leq x<b\}$ and that measure $\mu$ is uniquely determined by the measure $m$ on the real line. In fact, the measure $m$ is just the probability distribution of the function $\operatorname{Re}(z \cdot v) /|v|$ for any given $v \in \mathbb{C}^{n} \backslash\{0\}$. The uniqueness of the measure $\mu$ follows from the fact the computations of the moments of the probability distribution $m$ on $\mathbb{R}$ yields the values of all integrals of the form

$$
\int_{\mathbb{C}^{n}} z^{\alpha} \bar{z}^{\beta} d \mu
$$

(in terms of binomial coefficients and of the moments of the probability measure $m$ ). Since $\mu$ has compact support and polynomials are dense in the space of continuous functions on compact sets, the uniqueness follows.

Finally, the measure $\mu$ is rotationally invariant as it is the unique measure which satisfies a rotationally invariant condition.

For any $(A, B) \in\left(\mathbb{R}^{+}\right)^{n}$, let $T_{A, B}: \mathbb{C}^{n} \rightarrow \mathbb{C}^{n}$ the invertible affine map defined as follows: for all $\left(x_{1}, y_{1}, \ldots, x_{n}, y_{n}\right) \in \mathbb{C}^{n} \equiv \mathbb{R}^{2 n}$,

$$
T_{A, B}\left(x_{1}, y_{1}, \ldots, x_{n}, y_{n}\right)=\left(x_{1} / A_{1}, y_{1} / B_{1}, \ldots, x_{n} / A_{n}, y_{n} / B_{n}\right) .
$$

Lemma 5.7. Let $\mu$ be a compactly supported Borel probability measure on $\mathbb{C}^{n}$. Assume that there exists $(A, B) \in\left(\mathbb{R}^{+}\right)^{n} \times\left(\mathbb{R}^{+}\right)^{n}$ such that for any $a, b \in \mathbb{R}$ there exists a constant $m_{A, B}(a, b)$ such that, for all $(r, \theta) \in\left(\mathbb{R}^{+}\right)^{n} \times \mathbb{R}^{n}$,

$$
\mu\left\{z \in \mathbb{C}^{n} \mid a \leq \frac{\operatorname{Re}\left(\sum_{s=1}^{n} r_{s} e^{i \theta_{s}} z_{s}\right)}{\left[\sum_{s=1}^{n} r_{s}^{2}\left(A_{s}^{2} \cos ^{2} \theta_{s}+B_{s}^{2} \sin ^{2} \theta_{s}\right)\right]^{1 / 2}}<b\right\}=m_{A, B}(a, b) .
$$

Then the measure $\mu$ has the following form: there exists a rotationally invariant measure $\rho$ on $\mathbb{C}^{n}$ such that

$$
\mu=\left(T_{A, B}^{-1}\right)_{*}(\rho) .
$$

In fact, a stronger result holds. The key step is given by the following result.

Lemma 5.8. Let $\mu$ be a compactly supported Borel probability measure on $\mathbb{C}^{n}$. Let $(A, B) \in\left(\mathbb{R}^{+}\right)^{n} \times\left(\mathbb{R}^{+}\right)^{n}$ be a pair of vectors such that

$$
A_{1} \neq B_{1}, \quad \ldots, \quad A_{n} \neq B_{n} .
$$

If there exist distinct integral vector $v^{(1)}, \ldots, v^{(n)} \in \mathbb{Z}^{d}(d \geq 1)$ such that the probability distribution $m_{A, B, r, \theta}$ of the function

$$
\frac{\operatorname{Re}\left(\sum_{s=1}^{n} r_{s} e^{i<v^{(s)}, \theta>} z_{s}\right)}{\left[\sum_{s=1}^{n} r_{s}^{2}\left(A_{s}^{2} \cos ^{2}<v^{(s)}, \theta>+B_{s}^{2} \sin ^{2}<v^{(s)}, \theta>\right)\right]^{1 / 2}},
$$

defined on the probability space $\left(\mathbb{C}^{n}, \mu\right)$, is independent of $\theta \in \mathbb{T}^{d}$, for any given $r \in\left(\mathbb{R}^{+}\right)^{n}$, then it is also independent of $r \in \mathbb{R}^{n} \backslash\{0\}$, hence there exists a probability distribution $m_{A, B}$ on the real line such that

$$
m_{A, B, r, \theta}=m_{A, B}, \quad \text { for all }(r, \theta) \in \mathbb{R}^{n} \backslash\{0\} \times \mathbb{T}^{d} .
$$


Proof. By assumption there exists a compactly supported measure $m_{A, B, r}$ on the real line such that $m_{A, B, r, \theta}=m_{A, B, r}$ for all $\theta \in \mathbb{T}^{n}$. A computation of the moments $\mathcal{M}_{A, B, r}^{(k)}$ of the measure $m_{A, B, r}$ yields that all the odd moments $\mathcal{M}_{A, B, r}^{(2 k+1)}$ vanish while from the the computation of even moments $\mathcal{M}_{A, B, r}^{(2 k)}$ we can derive the identities below. Let $P_{A, B, r}(\theta)$ be the trigonometric polynomial

$$
P_{A, B, r}(\theta):=\sum_{s=1}^{n} r_{s}^{2}\left(A_{s}^{2} \cos ^{2}<v^{(s)}, \theta>+B_{s}^{2} \sin ^{2}<v^{(s)}, \theta>\right),
$$

which after a simple calculation can be written as follows:

$$
P_{A, B, r}(\theta)=\sum_{s=1}^{n} r_{s}^{2}\left(\frac{A_{s}^{2}-B_{s}^{2}}{4}\left(e^{2 i<v^{(s)}, \theta>}+e^{-2 i<v^{(s)}, \theta>}\right)+\frac{A_{s}^{2}+B_{s}^{2}}{2}\right) .
$$

The calculation of even moments yields:

$$
\int_{\mathbb{C}^{n}} \operatorname{Re}\left(\sum_{s=1}^{n} r_{s} e^{i<v^{(s)}, \theta>} z_{s}\right)^{2 k}=\mathcal{M}_{A, B, r}^{(2 k)} P_{A, B, r}(\theta)^{k} .
$$

Since by assumption the set of integral vectors $\left\{v^{(1)}, \ldots, v^{(n)}\right\}$ has distinct elements, it has a unique maximal element $v^{(l)}$ with respect to the lexicographic order on $\mathbb{Z}^{d}$. Thus by comparing the coefficients of the exponential $\exp \left(2 i k<v^{(l)}, \theta>\right)$ on the left and right sides of formula (104), it follows that

$$
r_{l}^{2 k} \int_{\mathbb{C}^{n}} z_{l}^{2 k} d \mu=\mathcal{M}_{A, B, r}^{(2 k)}\left(\frac{A_{l}^{2}-B_{l}^{2}}{2}\right)^{k} r_{l}^{2 k}
$$

By assumption $A_{l} \neq B_{l}$, hence on the set $\mathbb{R}^{n} \backslash\left\{r_{l}=0\right\}$ the moment $\mathcal{M}_{A, B, r}^{(2 k)}$ is given by the formula

$$
\mathcal{M}_{A, B, r}^{(2 k)}=\frac{2^{k} \int_{\mathbb{C}^{n}} z_{l}^{2 k} d \mu}{\left(A_{l}^{2}-B_{l}^{2}\right)^{k}} .
$$

It follows that the function $\mathcal{M}_{A, B, r}^{(2 k)}$ is constant on $\mathbb{R}^{n} \backslash\left\{r_{l}=0\right\}$. Since, by formula (104), it is continuous on $\mathbb{R}^{n} \backslash\{0\}$, it follows that $\mathcal{M}_{A, B, r}^{(2 k)}$ is equal to a constant $\mathcal{M}_{A, B}^{(2 k)}$ on $\mathbb{R}^{n} \backslash\{0\}$. Thus all the even moments $\mathcal{M}_{A, B, r}^{(2 k)}$ as well as all the odd moments $\mathcal{M}_{A, B, r}^{(2 k+1)}$ of the compactly supported probability measure $m_{A, B, r}$ do not depend on $r \in \mathbb{R}^{n} \backslash\{0\}$. It follows that there exists a probability distribution $m_{A, B}$ on the real line, with zero odd moments and even moments equal to $\mathcal{M}_{A, B}^{(2 k)}$, such that $m_{A, B, r, \theta}=m_{A, B}$ for all $(r, \theta) \in\left(\mathbb{R}^{n} \backslash\{0\}\right) \times \mathbb{T}^{d}$, as stated.

By Lemma 5.7 and Lemma 5.8, we can then derive the following characterization:

Lemma 5.9. Let $\mu$ be a compactly supported Borel probability measure on $\mathbb{C}^{n}$. Assume that there exists $(A, B, r) \in\left(\mathbb{R}^{+}\right)^{n} \times\left(\mathbb{R}^{+}\right)^{n} \times\left(\mathbb{R}^{+}\right)^{n}$ such that for any a, 
$b \in \mathbb{R}$ there exists a constant $m_{A, B, r}(a, b)$ such that, for all $\theta \in \mathbb{R}^{n}$,

$$
\mu\left\{z \in \mathbb{C}^{n} \mid a \leq \frac{\operatorname{Re}\left(\sum_{s=1}^{n} r_{s} e^{i \theta_{s}} z_{s}\right)}{\left[\sum_{s=1}^{n} r_{s}^{2}\left(A_{s}^{2} \cos ^{2} \theta_{s}+B_{s}^{2} \sin ^{2} \theta_{s}\right)\right]^{1 / 2}}<b\right\}=m_{A, B, r}(a, b) .
$$

If $A_{1} \neq B_{1}, \ldots, A_{n} \neq B_{n}$, then the measure $\mu$ has the following form: there exists a rotationally invariant measure $\rho$ on $\mathbb{C}^{n}$ such that

$$
\mu=\left(T_{A, B}^{-1}\right)_{*}(\rho) .
$$

\section{REFERENCES}

[1] A. Avila, M. Viana, Simplicity of Lyapunov Spectra: Proof of the Kontsevich-Zorich conjecture, Acta Math. 198, 2007, 1-56.

[2] V. Bargmann, Irreducible unitary representations of the Lorentz group, Ann. of Math. (2) 48, 1947, 568-640.

[3] P. Billingsley, Convergence of probability measures. 2nd ed. Wiley Series in Probability and Statistics. Chichester: Wiley, 1999.

[4] A. Bufetov, Finitely-additive measures on the asymptotic foliations of a Markov compactum, arXiv:0902.3303 1, 2009, 1-29.

[5] Limit Theorems for Translation Flows, arXiv:0804.3970v3, 2010, 1-69.

[6] __ Hölder cocycles and ergodic integrals for translation flows on flat surfaces, Electron. Res. Announc. Math. Sci. 17, 2010, 34-42.

[7] M. Burger, Horocycle flow on geometrically finite surfaces, Duke Math. J. 61, 1990, 779-803.

[8] F. Cellarosi, Limiting curlicue measures for theta sums. Ann. Inst. H. Poincaré Probab. Statist. 47, 2, 2011, 466-497.

[9] S. Cosentino, A note on Hölder regularity of invariant distributions for horocycle flows. Nonlinearity 18, 2005, 2715-2726.

[10] S. G. Dani. Invariant measures and minimal sets of horospherical flows. Invent. Math. 64, 1981, no. 2, 357-385.

[11] H. Fiedler, W. B. Jurkat \& O. Körner, Asymptotic expansions of finite theta series, Acta Arith. 32 (2), 1977, 129-146.

[12] L. Flaminio \& G. Forni, Invariant distributions and time averages for horocycle flows, Duke Math. J. 119, 2003, no. 3, 465-526.

[13] _ Equidistribution of nilows and applications to theta sums. Erg. Th. Dynam. Sys., 26:2, 2006, 409-433.

[14] S. V. Fomin, On generalized eigenfunctions of dynamical systems. (Russian) Uspehi Mat. Nauk (N.S.) 10, (1955). no. 1(63), 173-178.

[15] G. Forni, Solutions of the cohomological equation for area-preserving flows on compact surfaces of higher genus, Ann. of Math. 146, 1997, 295-344.

[16] Deviation of ergodic averages for area-preserving flows on surfaces of higher genus, Ann. of Math. 155, 2002, 1-103.

[17] H. Furstenberg, The unique ergodicity of the horocycle flow, in Recent Advances in Topological Dynamics (New Haven, Conn., 1972), Lecture Notes in Math. 318, Springer, Berlin, 1973, 95115.

[18] I. M. Gelfand \& S. V. Fomin, Unitary representations of Lie groups and geodesic flows on surfaces of constant negative curvature (in Russian), Dokl. Akad. Nauk SSSR 76, 1951, 771774.

[19] I. M. Gelfand \& M. Neumark, Unitary representations of the Lorentz group, Acad. Sci. USSR. J. Phys. 10, 1946, 93-94.

[20] B. M. Gurevich, The entropy of horocycle flows (in Russian), Dokl. Akad. Nauk SSSR 136, 1961, 768-770.

[21] G. A. Hedlund, Fuchsian groups and transitive horocycles, Duke Math. J. 2 , 1936, 530-542. 
[22] D. A. Hejhal, On the uniform equidistribution of long closed horocycles. Loo-Keng Hua: a great mathematician of the twentieth century. Asian J. Math. 4, 2000, no. 4, 839-853.

[23] S. Helgason, A duality for symmetric spaces with applications to group representations, Advances in Math. 5, 1970, 1-154.

[24] W. B. Jurkat \& J. W. Van Horne, On the central limit theorem for theta series. Michigan Math. J. 29 (1), 1982, 65-77.

[25] _ The uniform central limit theorem for theta sums. Duke Math. J. 50 (3), 1983, 649666.

[26] M. Kontsevich, Lyapunov exponents and Hodge theory, in "The mathematical beauty of physics", Saclay, 1996. Adv. Ser. Math. Phys. 24. World Scientific, River Edge, NJ, 1997, 318332.

[27] B. Marcus, The horocycle flow is mixing of all degrees, Invent. Math. 46, 1978, 201-209.

[28] J. Marklof, Limit theorems for theta sums, Duke Math. J. 97, 1999, 127-153.

[29] O. S. Parasyuk, Flows of horocycles on surfaces of constant negative curvature (in Russian), Uspekhi Mat. Nauk 8, no. 3, 1953, 125-126.

[30] M. Ratner, Factors of horocycle flows, Ergodic Theory Dynam. Systems 2, 1982, 465-489.

[31] _ Rigidity of horocycle flows, Ann. of Math. (2) 115, 1982, 597-614.

[32] _ Horocycle flows, joinings and rigidity of products, Ann. of Math. (2) 118,1983, 277313.

[33] _ The rate of mixing for geodesic and horocycle flows, Ergodic Theory Dynam. Systems 7, 1987, 267-288.

[34] P. Sarnak, Asymptotic behavior of periodic orbits of the horocycle flow and Eisenstein series, Comm. Pure Appl. Math. 34, 1981, 719-739.

[35] A. Strömbergsson, On the uniform equidistribution of long closed horocycles. Duke Math. J. $\mathbf{1 2 3}, 2004$, no. 3, 507-547

[36] D. Zagier, Eisenstein series and the Riemann zeta function, in Automorphic Forms, Representation Theory and Arithmetic (Bombay, 1979), Tata Inst. Fund. Res. Studies in Math. 10, Tata Inst. Fund. Res., Bombay, 1981, 275-301.

[37] A. Zorich, Asymptotic flag of an orientable measured foliation on a surface, in Geometric Study of Foliations, World Scientific, 1994, 479-498.

[38] , Finite Gauss measure on the space of interval exchange transformations. Lyapunov exponents, Ann. Inst. Fourier (Grenoble) 46, 1996, 325-370.

[39] , Deviation for interval exchange transformations, Ergod. Th. \& Dynam. Sys. 17, 1997, $1477-1499$.

Department of Differential Equations, Steklov Institute of Mathematics, Moscow, Russian Federation, Department of Mathematics, Rice University, Houston, TX USA

Department of Mathematics, University of Maryland, College Park, MD USA 